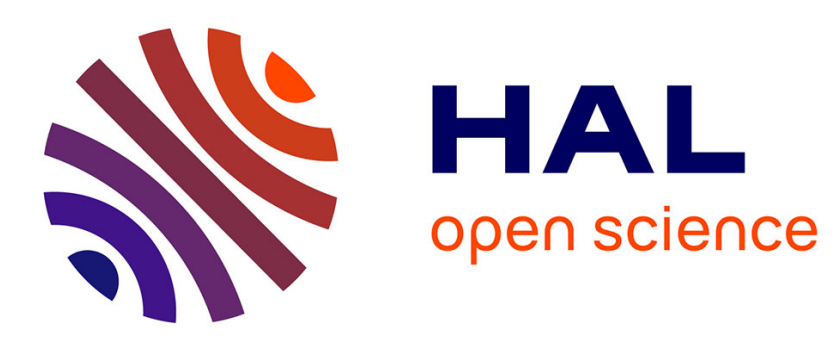

\title{
A One-Time Truncate and Encode Multiresolution Stochastic Framework
}

\author{
Remi Abgrall, Pietro Marco Congedo, Gianluca Geraci
}

\section{To cite this version:}

Remi Abgrall, Pietro Marco Congedo, Gianluca Geraci. A One-Time Truncate and Encode Multiresolution Stochastic Framework. [Research Report] RR-7967, INRIA Bordeaux, équipe CARDAMOM. 2012. hal-00699026v2

\section{HAL Id: hal-00699026 https://inria.hal.science/hal-00699026v2}

Submitted on 31 Aug 2015

HAL is a multi-disciplinary open access archive for the deposit and dissemination of scientific research documents, whether they are published or not. The documents may come from teaching and research institutions in France or abroad, or from public or private research centers.
L'archive ouverte pluridisciplinaire HAL, est destinée au dépôt et à la diffusion de documents scientifiques de niveau recherche, publiés ou non, émanant des établissements d'enseignement et de recherche français ou étrangers, des laboratoires publics ou privés. 
A One-Time Truncate and Encode

Multiresolution

Stochastic Framework

Remi Abgrall, Pietro Marco Congedo, Gianluca Geraci

RESEARCH

REPORT

$\mathrm{N}^{\circ} 7967$

August 27, 2015

Project-Team Bacchus 



\title{
Cnzián
}

\section{A One-Time Truncate and Encode Multiresolution Stochastic Framework}

\author{
Remi Abgrall, Pietro Marco Congedo, Gianluca Geraci \\ Project-Team Bacchus
}

Research Report $n^{\circ} 7967$ - August 27, 2015 - 48 pages

\begin{abstract}
In this work a novel adaptive strategy for stochastic problems, inspired to the classical Harten's framework, is presented. The proposed algorithm allows building, in a very general manner, stochastic numerical schemes starting from a whatever type of deterministic schemes and handling a large class of problems, from unsteady to discontinuous solutions. Its formulations permits to recover the same results concerning the interpolation theory of the classical multiresolution approach, but with an extension to uncertainty quantification problems. The interest of the present strategy is demonstrated by performing several numerical problems where different forms of uncertainty distributions are taken into account, such as discontinuous and unsteady custom-defined probability density functions. In addition to algebraic and ordinary differential equations, numerical results for the challenging 1D Kraichnan-Orszag are reported in terms of accuracy and convergence. Finally, a two degree-of-freedom aeroelastic model for a subsonic case is presented. Though quite simple, the model allows recovering some physical key aspect, on the fluid/structure interaction, thanks to the quasi-steady aerodynamic approximation employed. The injection of an uncertainty is chosen in order to obtain a complete parameterization of the mass matrix. All the numerical results are compared with respect to classical Monte Carlo solution and with a non-intrusive Polynomial Chaos method.
\end{abstract}

Key-words: Multiresolution, Uncertainty Quantification, Stochastic collocation, Adaptive grid. 


\section{Une méthode multi-résolution pour les problèmes de quantification des incertitudes}

Résumé : Cette étude concerne une stratégie adaptative s'inspirant des méthodes multi-résolution introduites par Harten, pour la résolution des problèmes stochastiques. L'algorithme proposé permet de construire des schémas numériques stochastiques pour résoudre des problèmes non-stationnaires en présence de discontinuité avec n'importe quelle fonction de densité de probabilité (pdf) des paramètres d'input. La formulation choisie permet d'obtenir les mêmes résultats de l'approche multi-résolution classique, mais en plus des problèmes de quantification de l'incertitude peuvent être prises en compte. L'intérêt de cette étude est démontré sur plusieurs cas-tests, prenant en compte des pdfs discontinues et instationnaires. En particulier, des problèmes régis par des équations différentielles algébriques et ordinaires (les équations mono-dimensionnelles dites de Kraichnan-Orszag) sont considérés. Finalement, un problème régi par un modèle aéroélastique avec deux degré de liberté est pris en compte. Tous les résultats numériques sont comparés avec les solutions obtenues par une méthode Monte Carlo et une méthode basée sur le Chaos Polynomial non-intrusif.

Mots-clés : Multi-résolution, quantification des incertitudes, méthodes de collocation, maillages adaptatives. 


\section{Introduction}

Nowadays, the prediction of the numerical simulations is a fundamental task to attain for the optimization and the control of engineering devices. However, estimating the confidence of a numerical simulation remains very challenging. In recent years, a strong effort has been devoted to develop efficient numerical methods for taking into account the randomness in the numerical simulations.

The most popular and known method for the uncertainty quantification (UQ) is the Monte Carlo. Its development led back to the research on the nuclear devices in the context of the Manhattan project and is due to Fermi, von Neumann and Ulam. This method is based on a stochastic procedure to represent realizations of the solution for which the statistic moments can be computed. Despite its solid mathematical framework it represents a very expensive approach for most practical application because it requires a great number of realizations. Several improved versions of the classical Monte Carlo method have been proposed in literature for increasing the convergence rate, see for instance the recent work presented in [1], but they still remain unfeasible for complex problems when the evaluation of samples is expensive, as in most engineering problems.

One of the most important class of methods for UQ is based on the Polynomial Chaos (PC) representation. In the original work of Wiener [2], the solution is expanded in a polynomial Hermite basis, the so-called homogeneous chaos expansion. In recent years, Xiu and Karniadakis [3] demonstrated that the optimal convergence, with respect to non Gaussian probability distributions, can be achieved if orthogonal basis are chosen following the so-called Wiener-Askey scheme. This leads to the well-known generalized Polynomial Chaos (gPC). Following the procedure introduced by Xiu and Karniadakis, i.e. employing the probability density function as a weight function for searching the orthogonal basis, an optimal expansion basis could be virtually obtained from every kind of pdf, see for details $[4,5]$. Actually the gPC is often used in combination with Galerkin projection [6] techniques following the idea of Ghanem and Spanos [7], who first extended the applications of the PC in combination with finite elements. The gPC is recognized as one of the most efficient techniques thanks to its exponential rate of convergence. However, problems with discontinuities in the random space can lead to slow convergence. Similarly long-time integration problems could be encountered [8], where this behavior is due to the modification in time of the statistic properties of the solution that induces an efficiency loss of the polynomial basis in time. Recently, Gerritsma [9] proposed a time-dependent generalized Polynomial Chaos scheme based on the research of a time varying optimal polynomial basis.

Another class of method for the UQ is based on the stochastic collocation (SC) approach [10]. This strategy is based on building interpolants (polynomial), of the same dimensionality of the stochastic space, in order to approximate the solution. In order to reduce the computational cost for high-dimension problems, these methods are often coupled to sparse grids techniques. The sparse grid strategy has been proposed by Smolyak [11] allowing interpolation of the function in a reduced subset of points with respect to the full tensorization set. This strategy is a cure against the so-called curse of dimensionality [12] problem, i.e. the exponential growth of the number of points with respect to the stochastic dimensions $[13,14]$.

Actually, handling a non-smooth behavior for high-dimension problems remains a very challenging issue. It is not completely solved even for low or moderate dimension problems. In the context of gPC schemes, Wan and Karniadakis introduced an adaptive class of methods for solving the discontinuity issues by using local basis functions, the multi-element generalized Polynomial Chaos (ME-gPC) [15]. This strategy deals with an adaptive decomposition of the domain on which local basis are employed. In order to treat discontinuous response surfaces, Le Maître et al. applied a multiresolution analysis to Galerkin projection schemes [16, 17]. This class of schemes relies on the projection of the uncertain data on a multi-wavelets basis consisting of piecewise polynomial (smooth) functions. This approach is shown to be very CPU demanding. Consequently, two cures are then explored in the context of adaptive methods: automatically refine the multi-wavelets basis or adaptively partitioning the domain.

More recently, unsteady stochastic problems have been solved by means of multi elements techniques, employing the collocation simplex method [18]. Also for these stochastic collocation methods, adaptive strategies have been proposed in order to tackle the discontinuity issues. In the work of Agarwall [19], an adaptive stochastic collocation method, based on the recursive splitting of the domain, has been proposed. In this case the splitting of the domain and the adaptivity is applied directly to the sparse grid basis. A sparse grid collocation strategy, based on piecewise multi-linear hierarchical basis functions, has been adopted by Ma and Zabaras [20] to recover the convergence loose by a global polynomial approximations in presence of discontinuities. 
Recently, Abgrall et al. [21, 22, 23] introduced a new class of finite volume schemes capable to deal with discontinuous problems both in the physical and stochastic space for shock-dominated flows. The so called semi-intrusive scheme (SI) exhibits promising results in term of accuracy and efficiency compared to more classical Monte Carlo and gPC methods. The idea is to extend to the stochastic space the finite volume representation used for the deterministic scheme. The established framework of the reconstruction techniques (ENO/WENO) in finite volume schemes can be, very easily, employed in the stochastic space with the SI scheme. This approach can lead to some advantages such as an extreme flexibility with respect the form of the pdf (that can be discontinuous and unsteady), an easy implementation, a slight modification of the deterministic solver preserving the number of equations.

The aim of the present work is to provide a framework, inspired to the classical multiresolution representation of Harten [24], capable to recover the same results of this theory but including new features for the extension to stochastic problems. The proposed algorithm, the Truncate and Encode (TE) strategy, displays very good properties in terms of convergence and efficiency. Moreover, it allows handling adaptively a stochastic mesh in a very general way. This could allow in the future a very easy coupling with different kinds of numerical methods as, for example SC and SI schemes. While in this work no dependence on the physical spaces is considered, the long-term objective is to build accurate numerical scheme, for low or moderate number of uncertainties, permitting to deal with unsteady discontinuous solutions and using unsteady refinement/derefinement capabilities both in the physical and stochastic space.

The approach proposed in the present work is based on a multiresolution concept, as already made in Le Maître's work. Anyway, the approach differs completely since here no spectral projection is employed, as it will be explained in the next section. Moreover, the possibility to reject a wavelets (equal to an interpolation error as in the original Harten framework) is based only on local tests, then is different from Galerkin projection approach where 1D energy estimator along stochastic dimensions are used. For details on the multiresolution approach applied to Galerkin projection schemes, the reader can refer to the extremely exhaustive reference [6].

The paper is organized as follows. In section 2, the classical multiresolution framework of Harten is illustrated. Some remarks are pointed out over the analytical results associated to the compression procedure. The algorithm proposed in this work, i.e. the Truncate and Encode (TE) strategy is presented in section 3, while the accuracy preserving time-advancement procedure in section 4. Differences between the classical MR framework and the present approach are discussed in section 5. Accuracy and efficiency of the the TE algorithm are verified against several numerical experiences in section 6. In particular, algebraic and ordinary differential equation (both scalar and vectorial) and the 1D Kraichnan-Orszag problem with uniform and discontinuous-unsteady probability distribution functions are considered. Finally, a two degree-of-freedom aeroelastic model is used to estimate the unsteady statistics for the motion. Remark that all the presented results are compared with respect to classical Monte Carlo methods and with gPC non intrusive approach. Some concluding remarks and perspectives are reported in section 7.

\section{Harten's Multiresolution framework}

In this section, we briefly recall the classical Harten MR framework [25, 24] extended here to the case of nonuniform measure. Consider a function $u(\xi)$ defined on a domain $\Xi=[0,1]$. Let us suppose to know the values $u^{0}=\left\{u_{j}^{0}\right\}_{j=0}^{N_{0}}$ on a uniform grid, defined as follows

$$
\mathcal{G}^{0}=\left\{\xi_{j}^{0}\right\}_{j=0}^{N_{0}}, \quad \xi_{j}^{0}=j h_{0}, \quad h_{0}=1 / N_{0}
$$

This grid is assumed as the finest one, i.e. the highest resolution level. Now, let us consider the set of nested dyadic grids $\mathcal{G}^{k}$ with $0 \leq k \leq L$

$$
\mathcal{G}^{k}=\left\{\xi_{j}^{k}\right\}_{j=0}^{N_{k}}, \quad \xi_{j}^{k}=j h_{k}, \quad h_{k}=2^{k} h_{0}, \quad N_{k}=N_{0} / 2^{k},
$$

where $k=0$ represents the finest level and $L$ the coarsest. Remark that the use of a nested dyadic structure allows obtaining a grid $\mathcal{G}^{k}$ from the finest adjacent level $\mathcal{G}^{k-1}$ by removing only the odd points and preserving the condition $\mathcal{G}^{k} \cap \mathcal{G}^{k-1}=\mathcal{G}^{k}$. The following relation holds

$$
u_{j}^{k}=u_{2 j}^{k-1} \text { for } \quad 0 \leq j \leq N_{k} .
$$


In this paper only structured uniform nested grids are employed, but the algorithm can be extended to an unstructured mesh as already shown in [26] provided that a nested structure is used between successive levels. The assumption of a regular mesh is then not exhaustive even in the 1D context. It is made here only for the sake of simplicity of exposure. The convention adopted in this work is reported in the figure 1.

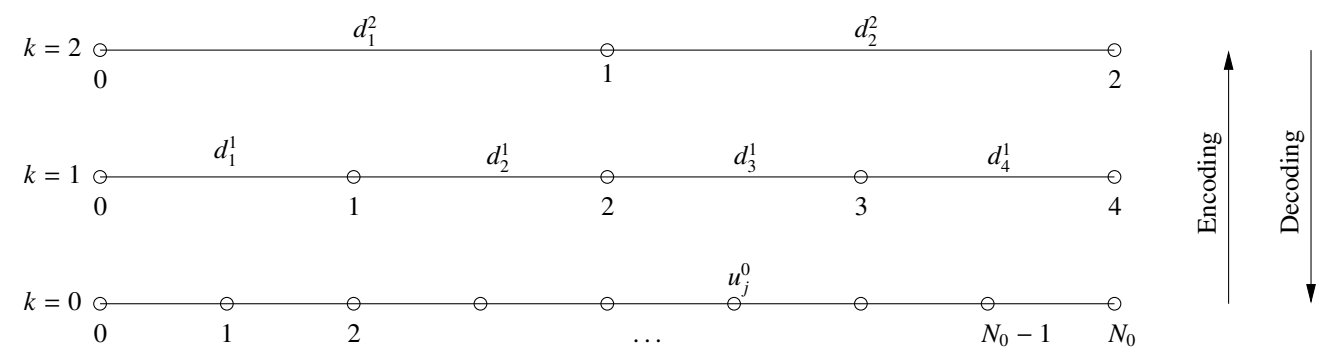

Figure 1: Harten's multiresolution classical notation.

In a general way an approximation problem can be solved on the mesh to obtain an interpolation operator $\mathcal{I}(\xi ; u)$ chosen to approximate the function $u$ at the coordinate $\xi$. The problem can be formulated (for the generic level $k$ th) as: find $\mathcal{I}_{j}\left(\xi ; u^{k}\right) \in \mathbb{P}^{r}(\Xi)$ such that for any point $\xi_{j}$ one has $L_{j}\left(\mathcal{I}\left(\xi ; u^{k}\right)\right)=L_{j}\left(u^{k}\right)$ for $0 \leq j \leq N_{k}$, where $\left\{L_{j}\right\}_{1 \leq j \leq r}$ is a family of linear forms defined on $C^{\bar{r}}(\mathbb{R})(\bar{r}>r)$. With this assumption two different kinds of linear forms could be considered:

- Lagrange interpolation: the linear form is directly equal to the point value of the function

$$
L_{j}\left(u^{k}\right)=u\left(\xi_{j}\right) \quad 0 \leq j \leq N_{k}
$$

- (Conditional) average reconstruction: in which at each point $\xi_{j}$ is associated its surrounded cell $\Xi_{j}$ such that

$$
L_{j}\left(u^{k}\right)=\bar{u}_{j}^{k}=\frac{\int_{\Xi_{j}} u^{k} \mathrm{~d} \xi}{\int_{\Xi_{j}} \mathrm{~d} \xi} .
$$

In the following often no distinction is made by the two reconstruction procedures, however in the case of constant reconstruction, employed in this work, the two procedures are equivalent (see the Appendix A ).

With a such operator $\mathcal{I}\left(\xi ; u^{k}\right)$ the values of the function (the average values) in the missing points, i.e. the points $\xi \in \mathcal{G}^{k-1}$ but not to $\mathcal{G}^{k} \cap \mathcal{G}^{k-1}$, can be obtained directly from the knowledge of the function at the level $k$ th

$$
\tilde{u}_{2 j-1}^{k-1}=\frac{\int_{\Xi_{2 j-1}^{k-1}} I_{2 j-1}^{k-1}\left(\xi ; u^{k}\right) \mathrm{d} \mu}{\int_{\Xi_{2 j-1}^{k-1}} \mathrm{~d} \mu} \text { with } \quad 1 \leq j \leq N_{k} .
$$

The operator $\mathcal{I}_{2 j-1}^{k-1}\left(\xi ; u^{k}\right)$ can be expressed as a function only of the values of the function at the level $k$ th (see the appendix Appendix A for the constant reconstruction example). The interpolation errors $d_{j}^{k}$ can be defined as follows

$$
d_{j}^{k}=\bar{u}_{2 j-1}^{k-1}-\tilde{u}_{2 j-1}^{k-1}, \quad \text { for } \quad 1 \leq j \leq N_{k},
$$

and generally they are called wavelets coefficients or details.

Remark that, if a constant piecewise reconstruction is used, the notation $u$ could indicate the values, point value or average value, without altering the previous formulation.

The MR strategy is based on the observation that the knowledge of the couple $\left(d^{k}, u^{k}\right)$, where $d^{k}=\left\{d_{j}^{k}\right\}$ and $u^{k}=\left\{u_{j}^{k}\right\}$, called multiresolution representation of $u^{k-1}$, permits to compute the solution on the grid $\mathcal{G}^{k-1}$. Of course, the viceversa also holds

$$
u^{k-1} \leftrightarrow\left(d^{k}, u^{k}\right)
$$


Proceeding recursively from $u^{0}$ to $u^{L}$

$$
u^{0} \leftrightarrow\left(d^{1}, u^{1}\right) \leftrightarrow\left(d^{1}, d^{2}, u^{2}\right) \leftrightarrow \cdots \leftrightarrow\left(d^{1}, d^{2}, \ldots, d^{L}, u^{L}\right) \stackrel{\text { def }}{=}\left(u_{M}\right)^{\mathrm{T}}
$$

it is possible to obtain the multi resolution representation of the solution $\left(u_{M}\right)^{\mathrm{T}}$.

Remark that it is possible to obtain the multiresolution representation $\left(u_{M}\right)^{\mathrm{T}}$ with any interpolation technique, i.e. any degree of interpolation is allowed provided the appropriate stencil. In this framework two different operations can be defined: the encoding procedure that allows obtaining the multiresolution representation from the knowledge of $u^{0}$ (the solution at the finest level) and the decoding procedure that allows obtaining the function at the finest resolution level from the multiresolution representation. If a matrix notation is employed the encoding procedure could be formulated as

$$
u_{M}=M u^{0},
$$

where $M$ is a $\left(N_{0}+1\right) \times\left(N_{0}+1\right)$ matrix. The decoding procedure is the inverse procedure, then obviously

$$
u^{0}=M^{-1} u_{M} .
$$

This matrix form is possible if the set of stencil is fixed and there is no adaptation, as for instance it happens in ENO reconstructions. In the case of automatic procedure to adapt the stencil the matrix $M$, that would not be linear, could be computed term by term in a closed form. The same is valid for the 'inverse' operator, see [27].

The MR framework, as presented in this section, could be seen only as hierarchical evaluation of a solution or, if applied to a numerical scheme, a hierarchical recasting of the same scheme. However one of the main advantages of the MR is the possibility to obtain more efficient schemes introducing a truncation procedure. The data compression capabilities of MR framework are then treated in the next section.

\subsection{Data compression}

As depicted in the previous section, the finest level can be reconstructed by the decoding procedure exploiting the multiresolution representation of the function, that is constituted by all the details $d_{j}^{k}$ and all the point values of the function $u_{j}^{k}$. If we consider the equation (3) rearranged in the following form

$$
u_{2 j-1}^{k-1}=d_{j}^{k}+\tilde{u}_{2 j-1}^{k-1},
$$

it is evident that the value of the function $u_{2 j-1}^{k-1}$ can be obtained directly from $\tilde{u}_{2 j-1}^{k-1}$ through the detail $d_{j}^{k}$. The approximate value is obtained by interpolation, as shown in (2), from the adjacent level.

The storage memory, required for the multiresolution representation, can be reduced using the following procedure. Let us choose a certain threshold $\varepsilon$. Then, the details can be compared to the threshold imposed for the $k$-th level $\varepsilon_{k}$, yielding a truncated details $\hat{d}_{j}^{k}$ defined as follows:

$$
\hat{d}_{j}^{k}=\left\{\begin{array}{lll}
d_{j}^{k} & \text { if } & \left|d_{j}^{k}\right|>\varepsilon_{k} \\
0 & \text { if } & \left|d_{j}^{k}\right| \leq \varepsilon_{k} .
\end{array}\right.
$$

If the procedure is recursively followed for each level, a new multiresolution representation is obtained, with a large number of zero details, that do not need to be stored

$$
\left.\hat{u_{M}}=\operatorname{tr}\left(u_{M}\right)=\left(\hat{d}^{1}, \hat{d}^{2}, \ldots, \hat{d}^{L}, u^{L}\right)\right) .
$$

If the result of the decoding procedure, after the truncation, is denoted as $\hat{u}^{0}$

$$
\hat{u}^{0}=M \hat{u_{M}}=M \operatorname{tr}\left(u_{M}\right)
$$

the following estimation holds (see [27] for a proof), both in the $L_{1}$ and $L_{\infty}$ norms

$$
\left\|u^{0}-\hat{u}^{0}\right\| \leq C \varepsilon,
$$

$R R n^{\circ} 7967$ 
where the constant $C$ is independent on the coarsest level $L$ and the local threshold $\varepsilon_{k}$ is defined as

$$
\varepsilon_{k}=\varepsilon / 2^{k}
$$

As a result, one needs only to fix a threshold $\varepsilon$ for the finest level $k=0$ and, moving from the finest to the coarsest, the threshold for the other levels is directly obtained from the finer one.

Details in this framework are of strong importance not only to compress data, but because they can be associated to the local regularity of the function. This is a well-known interpolation result [24]. Let us consider a function $u(\xi)$ that has, in a point $\bar{\xi}, p-1$ continuous derivatives and a jump discontinuity in the $p$ th derivative, then for $\xi_{j}^{k}$ near $\bar{\xi}$ it holds

$$
\hat{d}_{j}^{k} \sim \begin{cases}\left(h_{k}\right)^{p}\left[u^{(p)}\right] & \text { for } 0 \leq p \leq \bar{r} \\ \left(h_{k}\right)^{r}\left[u^{(\bar{r})}\right] & \text { for } p>\bar{r}\end{cases}
$$

where $\bar{r}$ is the order of accuracy of the interpolation ( $\bar{r}=2$ in this work) and [.] denotes the jump at the discontinuity. Therefore $\left|d_{2 j}^{k-1}\right| \approx 2^{-\bar{p}}\left|d_{j}^{k}\right|$, with $\bar{p}=\min (p, r)$, if the $k$ th level is fine enough.

This means that, in a smooth region, the wavelets decreases with a rate determined by the local regularity and by the order of the interpolation, while in a region near the discontinuity the wavelet remains of the same order for all the levels of refinement. This information could permit to build adaptive procedures for evaluating the numerical fluxes in the framework of finite volumes schemes. One could shift from the centered to non oscillatory schemes (ENO, WENO) in the neighborhood of a discontinuity [27]. In this work, this property is not exploited but it could be used to extend adaptivity of the stochastic space in the semi-intrusive method [21, 22, 23] recently proposed. However in this case the procedure could become highly non linear.

However, informations about the regularity of the function are the hearth of the adaptive procedure proposed in this work and that is presented in the next section.

\section{A one time Truncated-Encoding strategy}

The aim of this work is to develop a more flexible strategy, in the context of uncertainty quantification for compressible flow problems, in which the classical multiresolution framework $§ 2$ is employed as a basis to build a nonintrusive technique for steady and non-steady problems. However in the following $(\$ 4)$ a procedure to extend the present strategy to unsteady problems is also presented, introducing a proper advancing procedure, and some numerical results will be reported in $\S 6$.

A representation of the solution on a finest grid is computed starting from a coarsest grid, with a lower number of evaluation of the function. This implies that only a reduced set of point values, on the finest grid, is evaluated, while the remaining set is obtained by interpolation. This procedure moves recursively, with a combination of interpolation and evaluation, from the coarsest level to the finest. The direction here (from the coarsest to the finest) is the opposite with respect the classical Harten's framework, where, as shown in the previous section, the algorithm can start with an encoding procedure on the initial condition and successively the original system of equation is transformed into an equivalent set of equations on the wavelets coefficients. Then at each time step the scheme allows computing the solution on the finest level by the decoding procedure, i.e. the computation is explicitly performed only on the coarsest level and only the significative coefficients are computed.

Let us consider a scalar function $u=u(\xi)$ with $\xi \in \Xi=[0,1]$. The proposed strategy is constituted by the following steps (the notation is the same of the Harten's multiresolution framework, i.e $k=0$ for the finest level and $k=L$ for the coarsest):

- Initialization (the procedure can start only if the condition $m_{L}<m_{\max }$ is satisfied)

- Fix a threshold $\varepsilon$ (the solution is assumed to be solved with this threshold on the finest grid ${ }^{1}$ );

- Fix an index $m_{\max } \in \mathbb{N}$ for the maximum allowed level $\left(N_{\max }=N_{0}=2^{m_{\max }}\right)$;

- Fix an index $m_{L} \in \mathbb{N}$ for the coarsest level $\left(N_{L}=2^{m_{L}}\right)$.

${ }^{1}$ This is the same hypothesis as in the classical MR framework. 
- Evaluation of the function $u$ at each location at the coarsest level $u\left(\xi_{j}^{L}\right)=u_{j}^{L}$ with $j=0, \ldots, N_{L}$ where

$$
\mathcal{G}^{L}=\left\{\xi_{j}^{L}\right\}_{j=0}^{N_{L}}, \quad \xi_{j}^{L}=j h_{L}, \quad h_{L}=2^{L} h_{0}, \quad N_{L}=N_{0} / 2^{L},
$$

and $h_{0}=1 / N_{0}$. Each level can be labeled computing the equivalent index $k_{\text {eq }}$

$$
N_{k_{e q}}=\frac{N_{0}}{2^{k_{e q}}} \rightarrow 2^{k_{e q}}=\frac{N_{0}}{N_{k_{e q}}} \rightarrow \log _{2} 2^{k_{e q}}=k_{e q}=\log _{2}\left(\frac{N_{0}}{N_{k_{e q}}}\right),
$$

and then the coarsest level can be labeled by $k_{L}$ where $L=m_{\max }-m_{L}$.

- Evaluation of the subsequent level, with respect to the coarsest

$$
\mathcal{G}^{L-1}=\left\{\xi_{j}^{L-1}\right\}_{j=0}^{N_{L-1}}, \quad \xi_{j}^{L-1}=j h_{L-1}, \quad h_{L-1}=2^{L-1} h_{0}, \quad N_{L-1}=N_{0} / 2^{L-1} .
$$

- Starting of the adaptive strategy by means of a recursive procedure

A - The wavelets coefficients are computed for the present level $k$ as $^{2}$

$$
d_{j}^{k}=u_{j}^{k}-\frac{1}{2}\left(u_{\frac{j+1}{2}}^{k+1}+u_{\frac{j-1}{2}}^{k+1}\right) \quad \text { for } \quad 0 \leq j \leq N_{k} \quad \text { with } \quad j \text { odd }
$$

B - The wavelets coefficients are compared with the threshold $\varepsilon_{k}=\varepsilon / 2^{k}$. If $\left|d_{j}^{k}\right|>\varepsilon_{k}$ then the two nodes $\xi_{2 j+1}^{k-1}$ and $\xi_{2 j-1}^{k-1}$ will be flagged as active on the next finer mesh $\mathcal{G}^{k-1}$. If $\left|d_{j}^{k}\right|<\varepsilon_{k}$ then the wavelets is truncated, i.e. its value is posed zero.

$\mathrm{C}$ - The new level $k-1$ is generated if $k>0$ and only on the activated points the function $u$ is evaluated.

D - Moving from a level $k$ to the finer adjacent one $k-1$, three different cases are possible:

* If $\xi_{j}^{k} \in \mathcal{G}^{k} \cap \mathcal{G}^{k+1}$ then $u_{j}^{k}=u_{2 j}^{k+1}$ (shifting)

* If $\xi_{j}^{k} \notin \mathcal{G}^{k} \cap \mathcal{G}^{k+1}$ and it is not flagged then interpolate

$$
u_{j}^{k}=\frac{1}{2}\left(u_{\frac{j+1}{2}}^{k+1}+u_{\frac{j-1}{2}}^{k+1}\right)
$$

* If $\xi_{j}^{k} \notin \mathcal{G}^{k} \cap \mathcal{G}^{k+1}$ and it is flagged as active (by the step B of the algorithm) then evaluate, i.e. call the model.

E - The algorithm stops when the maximum level is reached or when all the wavelets coefficients can be truncated (at a certain level $k>0$ ).

In order to make things clearer, the application of the proposed strategy is illustrated on a triangular function $f=f(\xi)$ in figure 2. The following parameters, $m_{L}=0, m_{\max }=3$ then $L=m_{\max }-m_{L}=3$, are chosen. Full circles indicate an activated evaluation point, performed during the first steps of the algorithm when levels $k=3$ and $k=2$ are evaluated. Remark the exclusion of the shifted points of the level 2 from 3 . The interpolation operator is linear and the threshold is fixed. At the last (finest) level, the algorithm stops because the maximum level is reached, however no other points in level $k=0$ are marked as active and then the procedure would be stopped in any case. Remark that, at the level $k=1$, a square with a cross indicates an evaluated, but non activated point, in fact in this position the interpolation error is zero (the function is linear). The two neighborhood points at the next levels are then only interpolated (squares) and not evaluated by reducing the global computational cost.

\footnotetext{
${ }^{2}$ This is made here explicitly for the constant piecewise reconstruction but the same is still valid if the equation (2) is employed.
} 


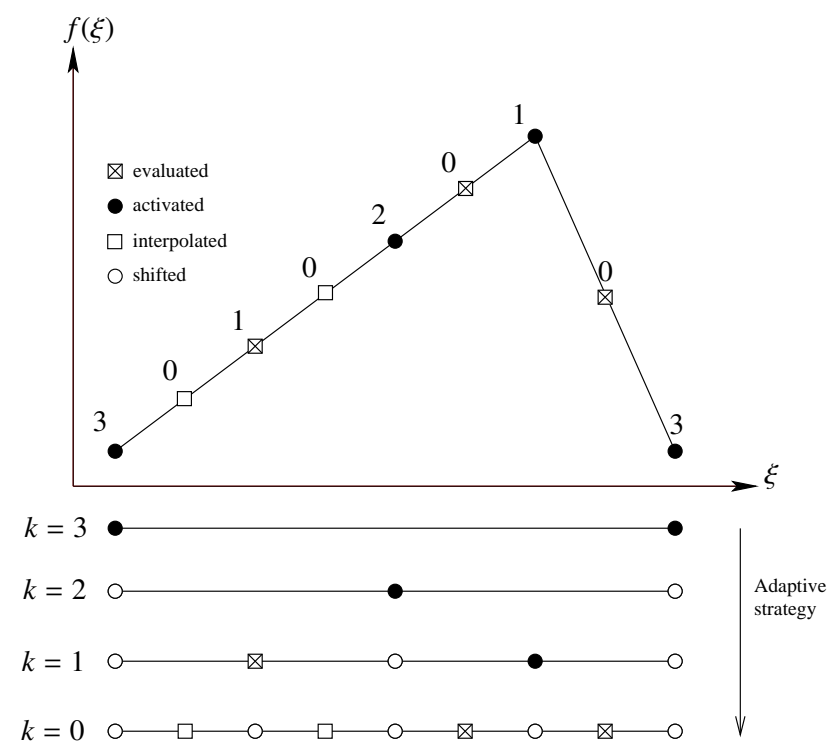

Figure 2: Example of application of the adaptive strategy on a triangular function $f=f(\xi)$.

\subsection{Application to the uncertainty quantification}

In the present work the case of equations with a solution depending only from the stochastic (and not physical) space is addressed. Due to the presence of a weight function, i.e. the probability density function, this case could be seen as a generalization of the classical approach in which function defined in the physical space (with unitary weight function) are considered.

One of the aim of UQ is to quantify the statistic moments of a quantity of interest. Let us assume to have a solution $u(\xi, t)$ for which the aim is to compute the statistics, i.e. for example the expectancy $\mathcal{E}$ :

$$
\mathcal{E}(t)=\int_{\Xi} u(\xi, t) p(\xi, t) \mathrm{d} \xi
$$

where $\xi \in \Xi$ is the parameter (or vector of parameters) and $p(\xi)$ is the probability density function (pdf) related to $\xi$. Remark that the pdf could assume a whatever form, including discontinuity and time-dependent properties.

Remark that the normalization condition, i.e. $\int_{\Xi} p(\xi, t) \mathrm{d} \xi=1$, must be always fulfilled for each pdf and time step.

The above integral could be computed when the conditional averages are already computed at the finest resolution level, i.e. $k=0$, by the following expression

$$
\mathcal{E}(t) \approx \sum_{i=0}^{N_{0}} \bar{u}\left(\xi_{i}^{0}, t\right) \mu\left(\Xi_{i}^{0}\right)
$$

where $\mu\left(\Xi_{i}^{0}\right)=\int_{\Xi_{i}^{0}} \mathrm{~d} \mu$ is the measure of the cell. Of course similar expressions can be derived for higher moments.

Now, let us show how the use of TE algorithm allows handling pdf's of whatever form with, eventually, traveling unsteady discontinuities in the stochastic space. Let us consider a solution $u(\xi)$ defined on the domain $\Xi=[-1,1]$ with a jump discontinuity located in $\xi=-1 / 2$ and smooth in the remaining part of the stochastic domain. If the pdf is uniform, the correct representation of the function, also in the neighborhood of its discontinuity becomes fundamental. However, let us imagine to have a different pdf distribution, for instance still an uniform pdf, but now defined only on the positive sub-domain $\Xi \supset \bar{\Xi}=[0,1]$, i.e. the pdf would be uniform and unitary in $[0,1]$ and equal to zero in $[-1,0]$. Despite the possibility to solve accurately the solution in the neighborhood of the discontinuity, this effort it is not motivated, because the weight function, i.e. the pdf, would lead to zero the contribution to the equation (11) in each point of the domain $[-1,0]$. Therefore, in the general case of a non smooth time-dependent pdf, it is more convenient 
to apply the TE algorithm to the product between the solution $u(\xi, t)$ and the pdf $p(\xi)$ considering a unitary measure $\mathrm{d} \mu=1 \mathrm{~d} \xi$. In particular the wavelet, for the level $k$, is computed as

$$
d_{j}^{k}=u_{j}^{k} p\left(\xi_{j}^{k}\right)-\widetilde{u_{j}^{k} p\left(\xi_{j}^{k}\right)} \quad \text { for } \quad 0 \leq j \leq N_{k} \quad \text { with } \quad j \text { odd }
$$

where $\widehat{u_{j}^{k} p\left(\xi_{j}^{k}\right)}$ is the approximated value for the product of the function $u$ with the pdf $p$ in the point $\xi_{j}^{k}$

$$
\widehat{u_{j}^{k} p\left(\xi_{j}^{k}\right)}=\frac{1}{\int_{\Xi_{j}^{k}} 1 \mathrm{~d} \xi} \int_{\Xi_{j}^{k}} \mathcal{I}\left(\xi ; u p^{k}\right) \mathrm{d} \xi
$$

Obviously in the case of piecewise constant reconstruction

$$
\widetilde{u_{j}^{k} p\left(\xi_{j}^{k}\right)}=\frac{1}{2}\left(u_{\frac{j+1}{2}}^{k+1} p\left(\xi_{\frac{j+1}{2}}^{k+1}\right)+u_{\frac{j-1}{2}}^{k+1} p\left(\xi_{\frac{j-1}{2}}^{k+1}\right)\right) \quad \text { for } \quad 0 \leq j \leq N_{k} \quad \text { with } \quad j \text { odd. }
$$

The computation of the wavelets should take into account the evolution, both in time and stochastic space, of the pdf and also of each component of a vectorial solution (if the system has several outputs). This can be performed by means of slight modifications of the TE algorithm, as presented in the next section.

\subsubsection{Application to vectorial solutions}

In this section, we focus our attention to a vectorial solution problem. Let us study the stochastic response of a system that has many outputs. In this case the application of the algorithm is straightforward if different runs of the algorithm are performed, one on each component of the response vector. The final adding step would be to merge together the two MR representations. Let us consider a vector of responses $u \in \mathbb{R}^{n}$ defined as $\mathbf{u}(\xi)=\left\{u_{1}(\xi), u_{2}(\xi), \ldots, u_{n}(\xi)\right\}^{\mathrm{T}}$. Only two modifications of the algorithm described in $\$ 3$ for a scalar function are to implement in order to handle vectorial solutions. Step A is formulated as follows: evaluate the wavelets coefficients $d_{j}^{k}$ for each element of $\mathbf{u}$ by introducing a new index $i$ for the component $u_{i}$ :

$$
d_{i, j}^{k}=u_{i, j}^{k}-\frac{1}{\int_{\Xi_{2 j-1}^{k-1}} \mathrm{~d} \mu} \int_{\Xi_{2 j-1}^{k-1}} I\left(\xi ; u_{i}^{k-1}\right) \mathrm{d} \mu \quad \text { for } \quad i=1, \ldots, n \quad \text { and } \quad 0 \leq j \leq N_{k} \quad(j \text { odd }) .
$$

The other modification concerns the step B where the criterion for the truncation is computed for all $d_{i, j}^{k}:\left|d_{i, j}^{k}\right|>\varepsilon_{k}$. If almost one of $d_{i, j}^{k}$ (for $\left.i=1, \ldots, n\right)$ cannot be truncated, i.e. $\left|d_{i, j}^{k}\right|>\varepsilon_{k}$, the two successive nodes $\left(\xi_{2 j+1}^{k-1}\right.$ and $\left.\xi_{2 j-1}^{k-1}\right)$ are activated at the subsequent level.

Remark that all the other steps remain the same presented for the scalar function and that for non-uniform distributed parameter the equation (12) should be employed.

At the end of the algorithm, the mesh that allows recovering the finest one with the prescribed accuracy regarding each component $u_{i}$ is obtained. Remark that in practical cases, this procedure, even if general, could not be very efficient. For example, when compressible fluid dynamics problem are considered (under the hypothesis of ideal gas), the density could be used as the unique parameter in order to detect smooth region, contact discontinuity and shock waves. In the section 6, this procedure has been applied to the 1D Kraichnan-Orszag problem and to an aeroelastic problem where the transverse deflection (that models the bending of the wing) and the torsional angle should be computed to recover the aerodynamic loads. In these cases, the TE algorithm has been applied on all the components following the procedure described in this section.

\section{An accuracy preserving time-advancement strategy}

In this section we focus on a procedure able to deal with time dependent probability density functions employing the TE algorithm. 


\subsection{Harten framework}

The original Harten framework provides a procedure based on updating the solution based on the CFL condition. The set of important coefficients is modified, at each time step, in order to capture the correct evolution of the solution. Then, the original numerical scheme can be reduced to a scheme on the wavelets coefficients [27].

Let us consider the case of linear interpolation. In this case, the multiresolution representation $u_{M}=\left\{d^{1}, d^{2}, \ldots, d^{L}, u_{L}\right\}^{\mathrm{T}}$ can be obtained multiplying the matrix $M$, of dimension $\left(N_{0}+1\right) \times\left(N_{0}+1\right)$ with the solution at the finest level $u_{0}$

$$
u_{M}(t)=M u_{0}(t)
$$

The time advancing procedure, used to obtain $u_{0}$ at the successive time steps, is based on the computation of the coarsest level $u_{L}$ and the wavelets coefficients $d^{k}$ with $k=1, \ldots, L$. These terms re-casted in the multiresolution vector can be used to compute the solution at the finest level

$$
u_{0}(t)=M^{-1} u_{M}(t)
$$

In the case of compressed values, i.e. the multiresolution representation after the truncation procedure, the procedure remains the same, but the multiresolution vector $u_{M}$ is truncated, then $\hat{u_{M}}$ is obtained from the truncated counterparts of the details (see the equation (7)).

As a result, the key part of the Harten algorithm is to identify and compute the important coefficients at each time step. This is made by a CFL based algorithm described in detail in [27]. The general idea is to identify the nodes in which an information can propagate and enlarge this stencil for stability reasons applying some empirical rules, the so-called safety set [27].

Many authors showed the efficiency of this procedure, but in the context of stochastic equations, finding a CFL condition can be hard. Then, it is not possible to apply directly this technique. In particular, the evolution of the probability density function is not governed by evolutionary equations. In the next section, we show how to extend the TE algorithm in order to follow the evolution of an unsteady pdf.

\subsection{Extension of TE algorithm to unsteady problems}

Let us assume an unsteady differential equation $\mathcal{L}(f(\xi))=0$ in which the random parameter has a probability distribution $p=p(\xi)$. The statistics of the output $f$ will be time-dependent according to the solution of the differential equation governed by the operator $\mathcal{L}$. In this case, the expectancy $\mathcal{E}$ should be computed as $\mathcal{E}(t)=\int_{\Xi} f(\xi, t) p(\xi) \mathrm{d} \xi$, then the multiresolution approach can be applied directly to $f(\xi, t)$ using a CFL-like condition. What happens if the output $f$ of the system is steady but the pdf is unsteady, i.e. $f=f(\xi)$ with $p=p(\xi, t)$ ? In this case the expectancy should be computed as $\mathcal{E}(t)=\int_{\Xi} f(\xi) p(\xi, t) \mathrm{d} \xi$. The pdf of the input could be imposed, but is not governed by a differential equation from which a CFL-like condition can be derived. In this case, the classical Harten framework can not be used. However, if the evolution of the product function $\tilde{f}(\xi, t)=f(\xi) p(\xi, t)$ is considered, an extended TE algorithm can be introduced.

The idea is to perform, for each time step, the TE algorithm on the product function $\tilde{f}(\xi, t)$. This allows minimizing the number of points to recover the finest mesh set of points, with an ad hoc interpolation technique, within a prescribed accuracy. At the successive steps, the integration in time could be performed starting from the knowledge of the solution at the previous step known with the prescribed tolerance.

Let us consider the solution of a Cauchy problem, i.e. find statistics for $y \in C(0, T)$

$$
\begin{cases}\dot{y}(t, \xi(t) & =f(t, y(t), \xi) \quad t \in[0, T] \quad \text { and } \quad \xi \in \Xi=[0,1] \\ y(0) & =y_{0} .\end{cases}
$$

The first step is to identify, as in the pure deterministic case, the time integration technique. If the time space is discretized as $t_{n}=n \Delta t$ with a constant step $\Delta t$ according to the stability condition, and if an explicit Euler scheme is considered, the equation (16) becomes

$$
y\left(t_{n+1}, \xi\right)=y\left(t_{n}, \xi\right)+\Delta t f\left(t_{n}, y\left(t_{n}\right), \xi\right)
$$


where each time integration is performed at a fixed location in the stochastic space. The TE algorithm must be, in the unsteady case, applied at each time step starting from the coarsest level to the finest one. According to the timeintegration technique, the choice of a stencil in time is needed in order to evaluate a solution in the point of the space $\Xi-t$. In the case of the explicit Euler scheme shown before, only the previous time step $t_{n}$ (at the same stochastic location $\xi$ ) is needed to evaluate the solution $y\left(t_{n+1}, \xi\right)$. For each location in the stochastic space $\xi_{j} \in \mathcal{G}^{k}=\left\{\xi_{j}^{k}\right\}_{j=0}^{N_{k}}$, corresponding to the stochastic mesh at the $k$ th level ${ }^{3}$ of the MR representation by the TE algorithm, two cases could occur:

1 At the previous time step the TE algorithm have reached a level equal or finer than the level $k$,

2 At the previous level a level coarser than $k$ has been reached.

In the first case the solution can be computed as

$$
y\left(t_{n+1}, \xi_{j}^{k}\right)=y\left(t_{n}, \xi_{j}^{k}\right)+\Delta t f\left(t_{n}, y\left(t_{n}\right), \xi_{j}^{k}\right)
$$

while in the second case the value $y\left(t_{n}, \xi_{j}^{k}\right)$ was not computed at the previous time step and it is not available to evaluate $y\left(t_{n+1}, \xi_{j}^{k}\right)$. However at each time step the representation of the solution, thanks to the TE algorithm, is known with a prescribed accuracy with respect the solution on the finest level as reported in the equation (8). The relation (8) justifies the possibility to interpolate the value of the function $y$ at the previous time step $y\left(t_{n}, \xi_{j}^{k}\right)$ as

$$
y\left(t_{n}, \xi_{j}^{k}\right) \approx \mathcal{I}\left(\xi_{j}^{k} ; y^{k}\left(t_{n}\right)\right)
$$

In the case of linear interpolation, considering the maximum level $\bar{k}$ reached at the time step $t_{n}$, the algorithm that can be used in order to compute the value of $y$ at the position $\xi_{j}^{k}$, is the following (see figure 3):

- Determination of the left value $\xi_{j_{L}}^{\bar{k}}$ as the maximum of the set $\left\{\xi_{j}^{\bar{k}} \mid \xi_{j}^{\bar{k}}<\xi_{j}^{k}\right\}$;

- Determination of the right value $\xi_{j_{R}}^{\bar{k}}$ as $\xi_{j_{R}}^{\bar{k}}=\xi_{j_{L}}^{\bar{k}}+h_{\bar{k}}$ where $h_{\bar{k}}=1 / 2^{\bar{k}}$.

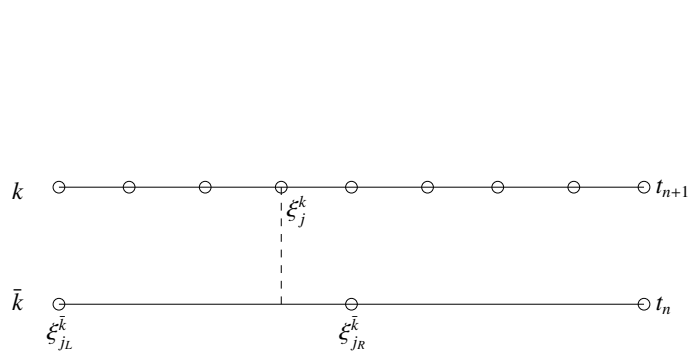

(a)

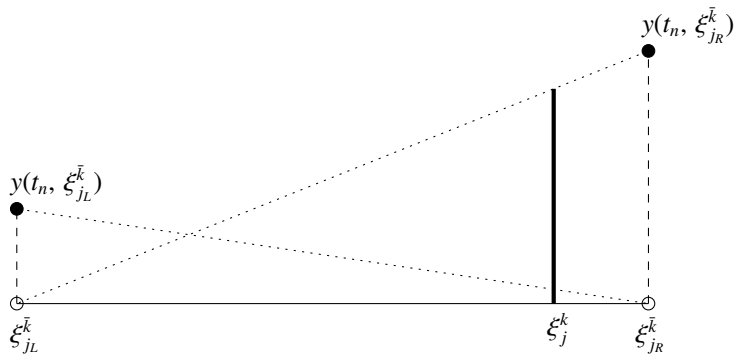

(b)

Figure 3: Linear interpolation stencil and convention adopted to perform the time integration. Stencil identification (a) and linear interpolation (b).

The value of the function $y$ at the time step $t_{n}$, interpolated by means of the values $y\left(t_{n}, \xi_{j_{L}}\right)$ and $y\left(t_{n}, \xi_{j_{R}}\right)$ already known, is then recovered by linear interpolation as

$$
y\left(t_{n}, \xi_{j}^{k}\right) \approx I\left(\xi_{j}^{k} ; y^{k}\left(t_{n}\right)\right)=\frac{y\left(t_{n}, \xi_{j_{R}}^{\bar{k}}\right)-y\left(t_{n}, \xi_{j_{L}}^{\bar{k}}\right)}{h_{\bar{k}}} \xi_{j}^{k}+y\left(t_{n}, \xi_{j_{L}}^{\bar{k}}\right)-j_{L}\left(y\left(t_{n}, \xi_{j_{R}}^{\bar{k}}\right)-y\left(t_{n}, \xi_{j_{L}}^{\bar{k}}\right)\right)
$$

where $j_{L}=\xi_{j_{L}}^{\bar{k}} / h_{\bar{k}}$. In the case of evaluation of the value $y\left(t_{n+1}, \xi_{j}^{k}\right)$, starting from the interpolated value at the previous time step, the scheme can be written as

$$
y\left(t_{n+1}, \xi_{j}^{k}\right)=\frac{y\left(t_{n}, \xi_{j_{R}}^{\bar{k}}\right)-y\left(t_{n}, \xi_{j_{L}}^{\bar{k}}\right)}{h_{\bar{k}}} \xi_{j}^{k}+y\left(t_{n}, \xi_{j_{L}}^{\bar{k}}\right)-j_{L}\left(y\left(t_{n}, \xi_{j_{R}}^{\bar{k}}\right)-y\left(t_{n}, \xi_{j_{L}}^{\bar{k}}\right)\right)+\Delta t f\left(t_{n}, \mathcal{I}\left(\xi_{j}^{k} ; y^{k}\left(t_{n}\right)\right), \xi_{j}^{k}\right)
$$

\footnotetext{
${ }^{3}$ The index of the level hereafter must be intended as equivalent indexes as presented in 3 .
} 
After some time steps the pattern of the different paths, corresponding to different stochastic location $\xi$, appear as a discontinuous succession of time integration. A typical pattern, for a Cauchy problem as the one considered in this section, employing an explicit Euler formula, is sketched in figure 4. An arrow means a time-advancement from a known, i.e. already computed point, while a bold arrow means an evaluation from an interpolated value.

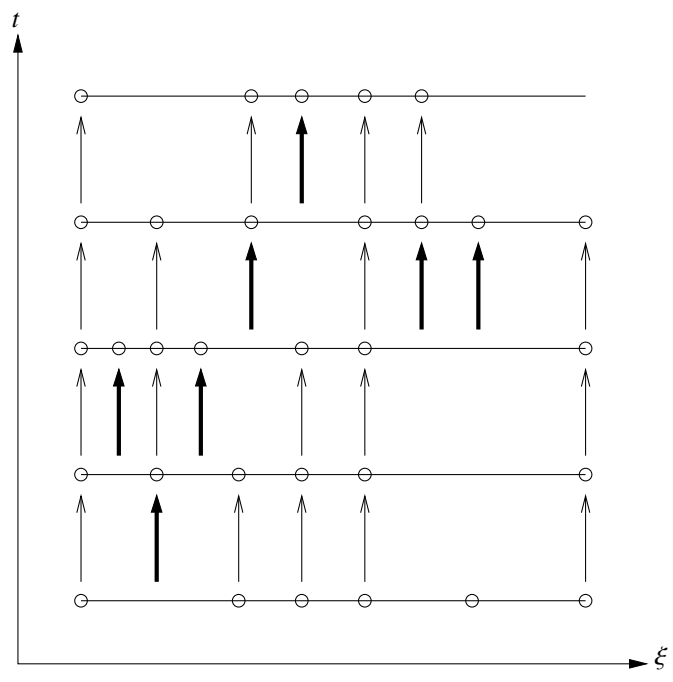

Figure 4: Time advancing. In bold the arrows for the advancing in time from interpolated value (equation (21))

We point out that this procedure permits to solve the long-time integration problem if a proper threshold is provided and if the number of refinements is large enough, according to the regularity of the solution. In the following, some results concerning the long-time integration problem will be illustrated for the 1D Kraichnan-Orszag problem.

The proposed time-advancement strategy can be easily used to build numerical scheme with automatic refinement/derefinement. The refinement/derefinement technique is shown in figure 4 where the time evolution, once fixed a certain position $\xi$, could not appear as a continuous sequence of advancing operations (arrows) on computed points (circles). It, instead, looks like a sequence of arrows and blank spaces (interpolation operations). This property is very promising for systems where unsteady discontinuities can appear only in a limited part of the domain, as for example in some compressible fluid dynamics problems.

\section{Some remarks on the difference between the classical and adaptive MR approach}

In this section, some differences between the classical approach discussed in $\$ 2$ and the adaptive procedure presented in $\S 3$ are illustrated. The TE algorithm is an encoding procedure with an embedded truncation capability. The application of the TE strategy produces a multiresolution representation $\hat{u_{M}}=\left(\hat{d}^{1}, \hat{d}^{2}, \ldots, \hat{d}^{L}, u^{L}\right)$, already truncated with respect to the accuracy governed by the threshold $\varepsilon$. This structure allows reconstructing the solution at the finer level $u^{0}$ starting from the coarsest level with the classical decoding procedure. Remark that in this case the encoding and decoding procedures move from the coarsest to the finest level, while in the classical MR approach the encoding procedure is performed starting from the finest level to the coarsest one.

Provided the differences between the two representations, one key question is if the multiresolution structures $u_{M}$ are or not the same. This condition is necessary to ensure that the norm estimation, i.e. equation (8), is guaranteed. In this work, a large number of numerical tests are performed in order to compare the multiresolution representations obtained with the classical and TE strategy. Different scalar functions with multiple discontinuities are taken into account. It was shown that if the coarsest level is fine enough, the two representations coincide. The required resolution (of the coarsest level) should be high enough in order to verify that, at the first refinement (always prescribed to initialize the algorithm), a point is located in the sharp region (if it exists). To make things simpler let us consider a function $f(x) \in[0,1]$ constituted by the sum of two scaled (unitary) Heaviside $H(x)$ functions

$$
f(x)=\mathrm{A} H(x-p)-\mathrm{A} H(x-(p+\delta))
$$


where A is a real positive constant (see figure 5). In this case, almost a point in the first two levels (the coarsest and the successive one) should fall in the domain $[p, p+\delta]$. Assuming a hierarchical nested sequence of uniform spaced grids, with no restriction related to the location $p$, this requirements reads: $h_{L-1}<\delta$, then the coarsest level should be equal to $h_{L}=2 h_{L-1}<2 \delta$. From the last relation, it follows that $N_{L}=1 / h_{L}=1 /(2 \delta)$. If this criterion is not satisfied, an error on the integration of the order $O(\delta)$ occurs.

Obviously this case is the worst one. Practically, a less strong condition is sufficient to ensure that the sharp region can be captured correctly by the TE algorithm and then the multiresolution representation coincides with the classical one.

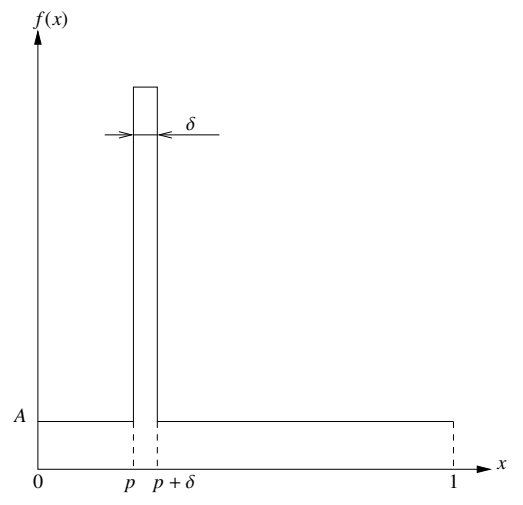

Figure 5: Example of a stiff function to reproduce with the TE algorithm due to a jump discontinuity located in a narrow support.

An important aspect to clarify remains why a novel and different MR approach is required in the context of stochastic differential equations. Let us consider the response of a system dependent from a random parameter (or a vector of parameter) in a steady configuration. The aim of this kind of analysis is the computation of statistics, for example the expectancy. To compute statistics it is necessary to (numerically) integrate the solution in the space of the parameters. Seeing the cost that can be associated to the computation of the solution in the space of the parameters (imagine the response of a complex numerical code), one issue of UQ is to reduce the computational cost of the global algorithm. In this case, the entire classical MR framework can not be used. In fact, from a theoretical point of view, it is always possible to compress a well-resolved solution, as for example in image processing applications, but this could be done only after the complete calculation at the finest level. For this reason, the TE algorithm is formulated, because it allows increasing computational efficiency preserving the same saving in term of memory requirement with respect the classical MR approach. In fact, the computation starts with the coarsest level and, only where required, the solution can be refined. At the end of the algorithm, the multiresolution representation will be the same as the classical MR framework, but the number of points in the space of parameters would be, hopefully, less.

This advantage is shown in figure 2. The classical framework would consist in nine computations, the entire finest level $k=0$, in order to obtain a multiresolution representation of four point (the two points at the coarsest level $k=3$ and the two wavelets activated at the levels $k=2,3$ ). The same results, in term of multiresolution representation, would be obtained by means of the TE algorithm with only seven computations (the full circles and crossed squares).

Classically the efficiency of the MR approach is measured by a compression ratio $\mu_{\mathrm{cr}}$ that is computed as the ratio between the number of points in the finest level $\left(N_{0}+1\right)$ and the number of significative wavelets $\left(N_{w}=\right.$ card $\left.\left\{d_{j}^{k}:\left|d_{j}^{k}\right|>\varepsilon_{k}, 0 \leq k \leq L-1\right\}\right)^{4}$, i.e. the number of active wavelets coefficients

$$
\mu_{\mathrm{cr}}=\frac{\left(N_{0}+1\right)}{N_{w}+\left(N_{L}+1\right)}
$$

For the case reported in figure 2 the compression ratio is $\mu_{\mathrm{cr}}=9 / 4$.

\footnotetext{
${ }^{4}$ Note that in the TE framework here presented, a wavelet $d_{j}^{k}$ is associated directly to the point $\xi_{j}^{k}$ at the finer level, i.e. the level at which the missing point is located, instead of the coarser level of the classical MR framework. This reflects on a different threshold $\varepsilon_{k}$ definition.
} 
This ratio is always the same for the classical approach and the present strategy as the MR representation is the same in both cases (see the discussion above). However, another ratio can be introduced, i.e. an evaluation compression ratio $\tau$, that measures the computational saving to obtain the MR strategy defined as the ratio between the number of points in the finer level and the number of evaluations needed $\left(N_{\text {eval }}=\operatorname{card}\left\{\xi_{j}^{k}: u\left(\xi_{j}^{k}\right)\right.\right.$ evaluated, $\left.\left.0 \leq k \leq L\right\}\right)$ to construct the MR representation

$$
\tau=\frac{\left(N_{0}+1\right)}{N_{\text {eval }}}
$$

In the previous example, reported in figure 2, the evaluation compression ratio is $\tau=1.29$. Of course, for the classical MR approach this ratio is always one because the solution must be known at the finest resolution level according to Harten's framework. Even if the compression capabilities of this strategy is the same than the classical approach, in this way it is possible to reduce computational cost for non-necessary functional evaluations. This is a very important property for treating UQ problems where a functional evaluation can be associated to a high computational cost. It could be useful to remark here that the TE algorithm differs significantly from other adaptive refinement techniques like, for example, the automatic mesh refinement (AMR) techniques. In the MR context, the solution is refined and an accuracy requirement is fulfilled, not only locally as in AMR, but with respect to the representation at a specific level (the finest). This is the key idea that allows building the time-advancement algorithm described in section 4.

Let us consider now unsteady problems. In this case, the MR classical framework seems to work properly. At the first time-step, the MR algorithm could obtain a multiresolution representation of the initial solution and, then, the adding cost related to non-necessary evaluations would be very small if the initial solution is known analytically. For the subsequent time-steps, the first to be really computed, some differences could exist between the MR classical approach and the present one. In fact, as already explained in the previous section, the MR strategy is based on a CFL approach for moving the significative wavelets. Then, if the problem is dominated by an unsteady pdf, the algorithm could move points basing only on the temporal evolution of the solution and not on the evolution of the product with the pdf. For this reason, the classical MR algorithm could totally fail having no CFL condition to follow. For this reason the time-advancement algorithm, presented in the previous section, is of great interest for unsteady pdf. A specific test-case is presented in the section 6.

\section{Numerical Results}

In this section, the TE algorithm is applied to several numerical test-cases in order to check its accuracy and convergence rate with respect to some classical stochastic methods, such as quasi-Monte Carlo and Polynomial Chaos. In the case of probability distribution not belonging the so-called Wiener-Askey scheme [3], in the following results a PC method has been employed to evaluate the statistics as in a collocation method (the function is multiplied by the pdf). However in the following we refer to this method generically as a Polynomial Chaos method. First, some steady algebraic problems $(\$ 6.1)$ are considered where analytical discontinuous functions are evaluated in terms of their expectancy and variance; for this case the performances in terms of compression and evaluation ratios are also evaluated. The capability of the TE algorithm to preserve accuracy for time-evolving solutions is displayed in two test-cases. The first-one (see Section §6.2) deals with an ordinary differential equation (ODE), i.e. taken from [6] but with some modifications in order to obtain a more stiff $\xi-t$ pattern of the function. The second test-case (see Section $\S 6.3$ ) for checking the convergence properties in long-time integration problem is the so-called Kraichnan-Orszag 1D ODE, a well-known problem in literature for testing UQ methods properties. Different kinds of pdf, i.e. uniform and discontinuous, are considered. Finally, a simplified model for aeroelastic study ( $\$ 6.6)$, a two degree-of-freedom typical wing section coupled with a quasi-steady strip theory model for aerodynamics, is proposed to compute the statistics of the motion considering uncertainties on mass properties for discontinuous probability distribution. For all the examples, exhaustive comparisons with quasi-Monte Carlo and Polynomial Chaos are performed.

\subsection{Steady problems}

Let us consider a function of the form $f=f(\xi)$, where the parameter $\xi \in \Xi$ takes uniform values between 0 and 1 , i.e. $\xi \sim \mathcal{U}[0,1]$. The aim is to compute expectancy $\mathcal{E}$ and variance Var for $f$ according to the following definitions

$$
\begin{aligned}
\mathcal{E} & =\int_{\Xi} f(\xi) p(\xi) \mathrm{d} \xi \\
\text { Var } & =\int_{\Xi}(f(\xi)-\mathcal{E})^{2} p(\xi) \mathrm{d} \xi .
\end{aligned}
$$


All the numerical integrals are computed with the trapezoidal rule on the points distribution generated by means of the TE algorithm. Results are compared with respect to the reference-solutions obtained from Monte Carlo and Polynomial Chaos methods.

Two model functions, with one or two discontinuities in the stochastic space, are considered. The function $f_{1}$ (see figure 6(a)), is a piecewise smooth function with one discontinuity located at $\xi=2 / 5$

$$
f_{1}(\xi)=\left\{\begin{array}{lr}
-\frac{15}{2} \xi^{2}+\frac{11}{2} \xi & 0 \leq \xi \leq 2 / 5 \\
-\frac{35}{12} \xi^{2}+\frac{13}{4} \xi-\frac{1}{3} & 2 / 5<\xi \leq 1 .
\end{array}\right.
$$

The second function $f_{2}$ (see figure $8(\mathrm{a})$ ) is constituted by a sinus function and a fourth-order polynomial form, then contains two discontinuities at $\xi=1 / 5$ and $\xi=3 / 4$ :

$$
f_{2}(\xi)= \begin{cases}10 \sin (\xi \pi) & \xi \leq 1 / 2 \text { or } \quad \xi \geq 3 / 4 \\ 10 \xi^{4}+79 / 4 & \text { otherwise }\end{cases}
$$

Starting with the coarsest level $m_{L}=1$ and with a maximum level between 3 and 12, using a threshold equal to $\varepsilon=10^{-1}$, the TE algorithm is used to generate the points distribution. The MC method is used to generate referencevalues for the expectancy and the variance with a number of points bounded between 5 and 101, while a PC approach is employed to evaluate the integrals with a degree between 5 and 100 with a step equal to 5 . The results are evaluated in terms of percentage errors, computed as follows

$$
\begin{aligned}
\operatorname{err}_{\mathcal{E}} & =\frac{\left|\mathcal{E}-\mathcal{E}_{\text {exact }}\right|}{\mathcal{E}_{\text {exact }}} 100 \\
\operatorname{err}_{\text {Var }} & =\frac{\mid \text { Var }- \text { Var }}{\text { Vaxact }} \mid \\
\text { rexact }_{\text {exa }} & 100
\end{aligned}
$$

In figure 7, we reported the errors for the mean $\mathcal{E}$ and for the variance Var with respect to the total amount of evaluations required $N$ to compute statistics on the left and on the right, respectively.

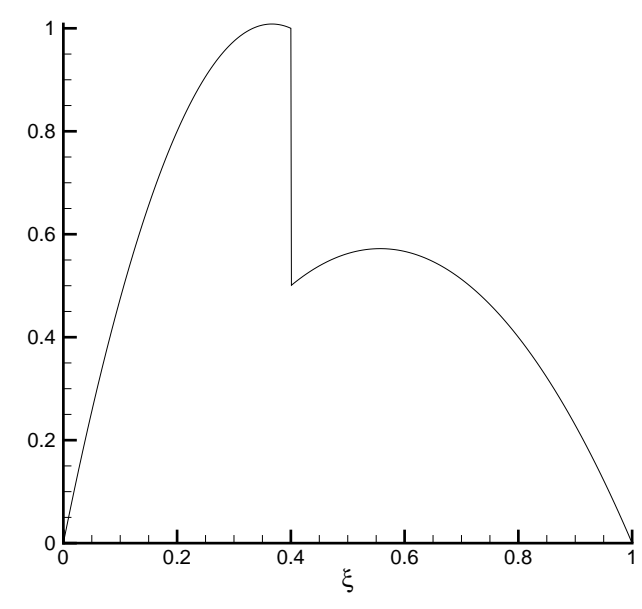

(a)

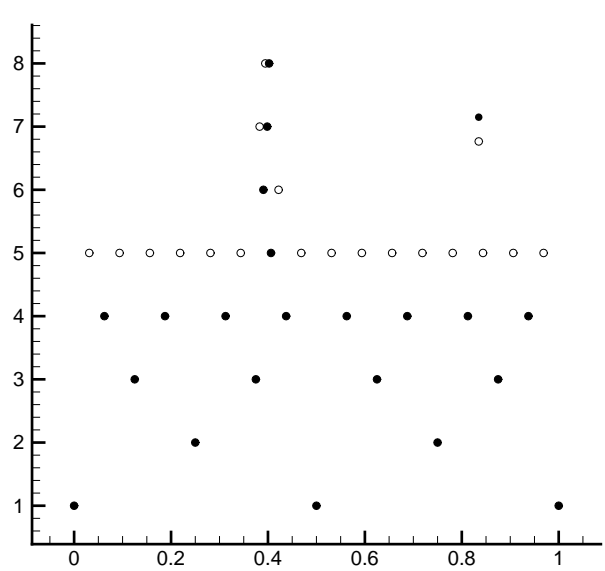

(b)

Figure 6: Model function $f_{1}$ (a) with its MR representation on the left (b).

All the methods exhibit a non monotone convergence to the exact value both for $\mathcal{E}$ and Var. The present MR algorithm allows reaching lower levels of error with a fixed number of evaluations $N$ and, moreover, allows obtaining the smoothest decrease of the error with the number of evaluations both for mean and variance. The MC method 
displays strong oscillations with respect to all the other methods while the PC exhibit a faster convergence with respect to the MC, but is affected by numerous oscillations.

In figure 7, the results of applying the trapezoidal rule without the TE algorithm, are also reported. It is possible to appreciate that the good performances obtained with TE are not related to the choice of quadrature formula (seeing that trapezoidal rule is not so accurate), but the efficiency in terms of computational cost can be attributed globally to the proposed algorithm. The trapezoidal rule results display several oscillations even if the errors are lower with respect to both the MC and PC methods.

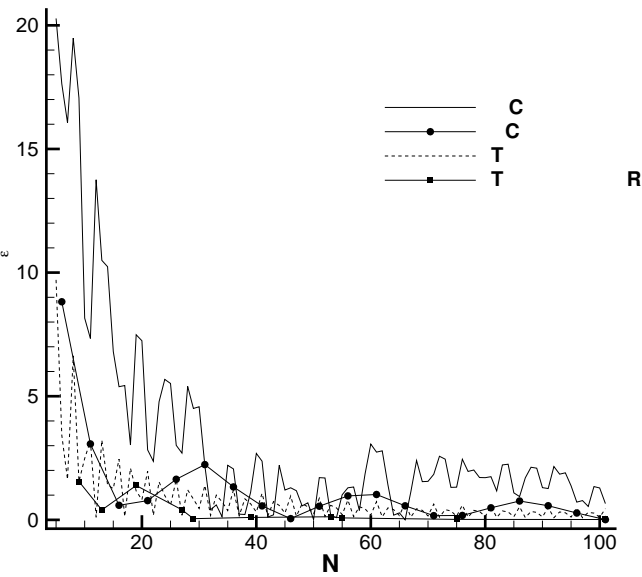

(a)

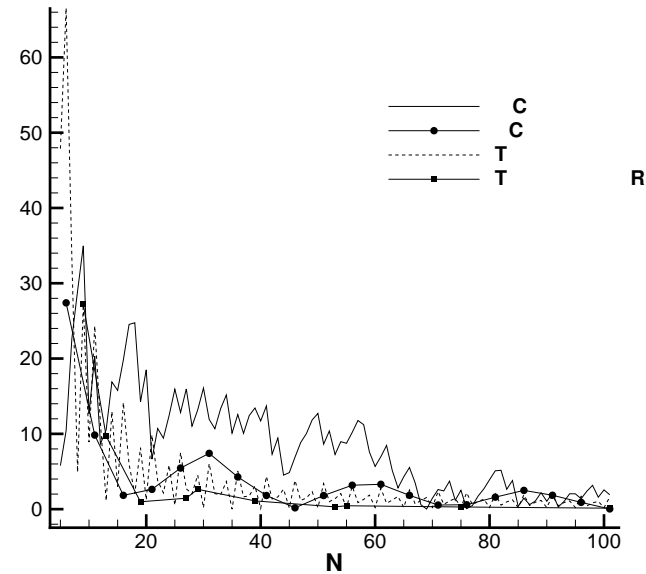

(b)

Figure 7: Errors for the mean (a) and variance (b) for the first function $f_{1}$.

To better understand the TE algorithm, in figure (b), the pattern of the computed points and the activated wavelets are reported. The maximum level was fixed, in this case, to $m_{\max }=8$ equal to 257 evaluations. As shown in figure (b), the adaptive algorithm do activate all the points until to the third refinement. At the fourth refinement, the accuracy requirement $\left(\varepsilon=10^{-1}\right)$ is reached for all the points but not for the point closer to the discontinuity. Then, the algorithm stops after that the maximum level is reached. It is remarkable that the stored points in figure 6(b) are the set of wavelets of the Harten framework after the truncation procedure. However, the TE algorithm needs some extra points to generate the MR representation, i.e. the wavelets not activated (the withe circles). At the end of the procedure, the compression ratio $\mu_{\mathrm{cr}}$ is equal to $\mu_{\mathrm{cr}}=257 / 21=12.24$. Moreover, the TE algorithm allows saving computational cost reaching the MR representation without knowing all the points at the maximum level $\left(2^{8}+1=257\right.$ in this case). The evaluation ratio $\tau$ is equal to $\tau=257 / 39=6.59$.

The table 1 summarizes the results obtained for the function $f_{1}$ starting with a coarsest level equal to 1 and with a maximum level between 3 and 12. Errors in the norms $L_{1}$ and $L_{\infty}$ are reported in terms of the number of the activated wavelets $N_{w}$, the number of the evaluated points $N_{\text {eval }}$ and the compression $\mu_{\text {cr }}$ and evaluation $\tau$ ratios. Errors in the norms $L_{1}$ and $L_{\infty}$ are computed as follows

$$
\begin{aligned}
& \operatorname{err}_{L_{1}}=\left\|f^{0}-\hat{f}\right\|_{L_{1}}=\frac{1}{N}\left|f_{i}^{0}-\hat{f}_{i}\right| \\
& \operatorname{err}_{L_{\infty}}=\left\|f^{0}-\hat{f}\right\|_{L_{\infty}}=\max _{i}\left|f_{i}^{0}-\hat{f}_{i}\right|,
\end{aligned}
$$

where $f^{0}$ is the function at the finest level $k=0$ and $\hat{f}$ is the truncated function, i.e. the function evaluated only in the set of points corresponding to the activated wavelets.

For the equation $f_{2}$ (see equation (26)), the pattern of the MR representation is reported in figure 8 (b) using a threshold equal to $\varepsilon=10^{-1}$ and the coarsest and the maximum level equal to 1 and 8 , respectively. Remark that The $\mathrm{TE}$ algorithm follows the two discontinuities where more points are generated until reaching the maximum level. The 


\begin{tabular}{c|c|c|c|c|c|c}
$m_{\max }$ & $N_{\mathrm{w}}$ & $N_{\mathrm{eval}}$ & $\mu_{\mathrm{cr}}$ & $\tau$ & err $L_{1}$ & err $L_{\infty}$ \\
\hline \hline 3 & 7 & 9 & $0.1285 \mathrm{E}+01$ & $0.1000 \mathrm{E}+01$ & $0.3331 \mathrm{E}-15$ & $0.9252 \mathrm{E}-16$ \\
4 & 8 & 13 & $0.2125 \mathrm{E}+01$ & $0.1308 \mathrm{E}+01$ & $0.1139 \mathrm{E}-01$ & $0.2681 \mathrm{E}-02$ \\
5 & 11 & 19 & $0.3000 \mathrm{E}+01$ & $0.1737 \mathrm{E}+01$ & $0.7324 \mathrm{E}-02$ & $0.2022 \mathrm{E}-02$ \\
6 & 15 & 27 & $0.4333 \mathrm{E}+01$ & $0.2407 \mathrm{E}+01$ & $0.2848 \mathrm{E}-02$ & $0.1236 \mathrm{E}-02$ \\
7 & 16 & 29 & $0.8063 \mathrm{E}+01$ & $0.4448 \mathrm{E}+01$ & $0.2848 \mathrm{E}-02$ & $0.1384 \mathrm{E}-02$ \\
8 & 21 & 39 & $0.1224 \mathrm{E}+02$ & $0.6590 \mathrm{E}+01$ & $0.1831 \mathrm{E}-02$ & $0.7150 \mathrm{E}-03$ \\
9 & 28 & 53 & $0.1832 \mathrm{E}+02$ & $0.9679 \mathrm{E}+01$ & $0.7121 \mathrm{E}-03$ & $0.3828 \mathrm{E}-03$ \\
10 & 29 & 55 & $0.3534 \mathrm{E}+02$ & $0.1863 \mathrm{E}+02$ & $0.7121 \mathrm{E}-03$ & $0.3854 \mathrm{E}-03$ \\
11 & 39 & 75 & $0.5254 \mathrm{E}+02$ & $0.2732 \mathrm{E}+02$ & $0.4578 \mathrm{E}-03$ & $0.1860 \mathrm{E}-03$ \\
12 & 52 & 101 & $0.7879 \mathrm{E}+02$ & $0.4056 \mathrm{E}+02$ & $0.1780 \mathrm{E}-03$ & $0.1004 \mathrm{E}-03$
\end{tabular}

Table 1: Results for the function $f_{1}$. The compression ratio $\mu_{\mathrm{cr}}$ and the evaluation compression ratio $\tau$ are computed as shown in the equations (23) and (24), respectively.

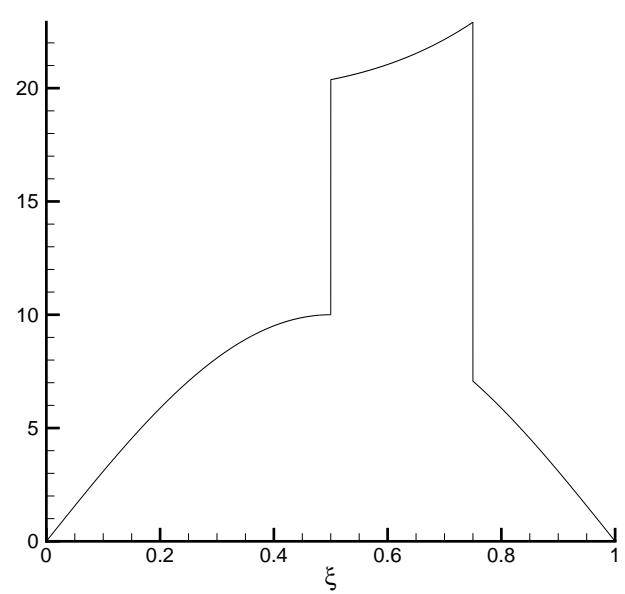

(a)

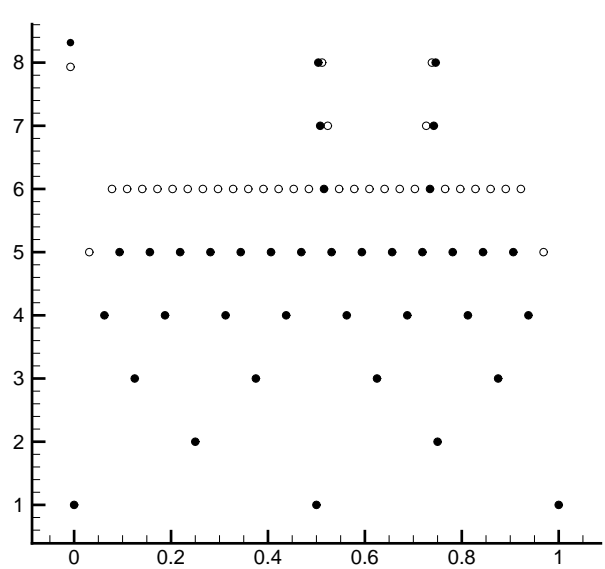

(b)

Figure 8: Function $f_{2}$ (a) and its MR representation (b).

advantage of such distribution of points can be clearly seen in the figure 9 , where the percentage errors for the mean $\mathcal{E}$ and variance Var are reported.

Also in this case the error displays strong oscillations, but the TE algorithm shows a monotone decrease of the errors with respect to the number of evaluations. For the other methods, convergence is attained only in terms of mean values. Even in this case, the effect of the TE algorithm in the points distribution allows obtaining better results with respect to the same trapezoidal quadrature technique. The detailed results in term of compression $\mu_{\mathrm{cr}}$ and evaluation $\tau$ ratios and the corresponding errors in norm $L_{1}$ and $L_{\infty}$ are reported in table 2.

In the next section some unsteady problems with continuous and discontinuous pdf are taken into account.

\subsection{A scalar ordinary differential equation}

In this section, the time-advancing strategy presented in $\$ 4$ is applied to some ordinary differential equations. In this section, the scalar case is analyzed, while the results for the vectorial cases are reported in $\$ 6.3$ and $\S 6.6$.

The first ODE example is extracted from [6] with some slight modifications in order to achieve a variable final 


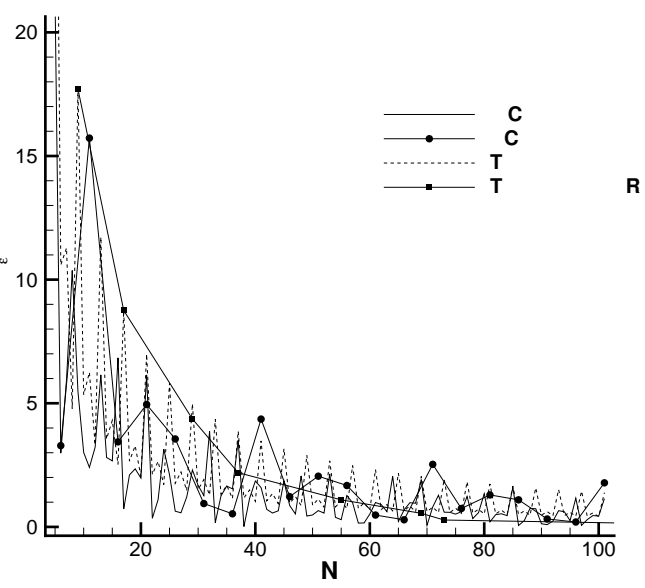

(a)

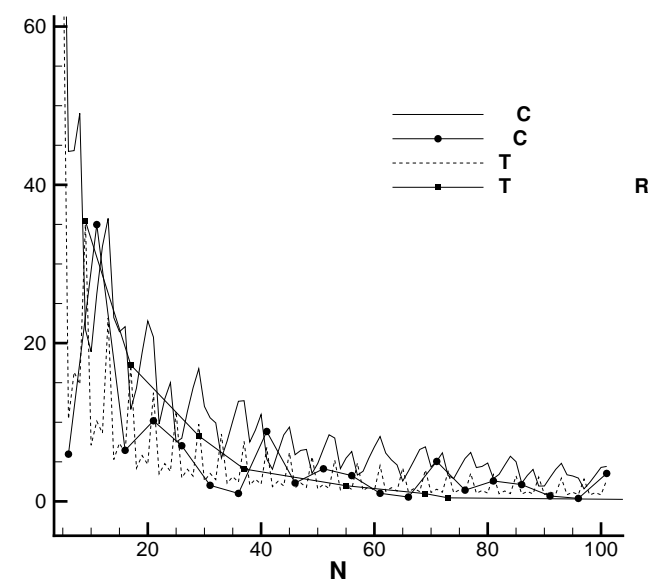

(b)

Figure 9: Errors of the mean (a) and variance (b) for the second function $f_{2}$.

\begin{tabular}{c|c|c|c|c|c|c}
$m_{\max }$ & $N_{\mathrm{w}}$ & $N_{\mathrm{eval}}$ & $\mu_{\mathrm{cr}}$ & $\tau$ & err $L_{1}$ & err $L_{\infty}$ \\
\hline \hline 3 & 9 & 9 & $0.1000 \mathrm{E}+01$ & $0.1000 \mathrm{E}+01$ & $0.3997 \mathrm{E}-14$ & $0.1283 \mathrm{E}-14$ \\
4 & 15 & 17 & $0.1133 \mathrm{E}+01$ & $0.1000 \mathrm{E}+01$ & $0.2487 \mathrm{E}-13$ & $0.4415 \mathrm{E}-14$ \\
5 & 17 & 29 & $0.1941 \mathrm{E}+01$ & $0.1138 \mathrm{E}+01$ & $0.1398 \mathrm{E}-01$ & $0.1133 \mathrm{E}-02$ \\
6 & 21 & 37 & $0.3095 \mathrm{E}+01$ & $0.1757 \mathrm{E}+01$ & $0.1203 \mathrm{E}-01$ & $0.2795 \mathrm{E}-02$ \\
7 & 30 & 55 & $0.4300 \mathrm{E}+01$ & $0.2345 \mathrm{E}+01$ & $0.6193 \mathrm{E}-02$ & $0.1551 \mathrm{E}-02$ \\
8 & 37 & 69 & $0.6946 \mathrm{E}+01$ & $0.3725 \mathrm{E}+01$ & $0.3011 \mathrm{E}-02$ & $0.1019 \mathrm{E}-02$ \\
9 & 39 & 73 & $0.1315 \mathrm{E}+02$ & $0.7027 \mathrm{E}+01$ & $0.3011 \mathrm{E}-02$ & $0.1070 \mathrm{E}-02$ \\
10 & 57 & 109 & $0.1798 \mathrm{E}+02$ & $0.9404 \mathrm{E}+01$ & $0.1611 \mathrm{E}-02$ & $0.4983 \mathrm{E}-03$ \\
11 & 69 & 133 & $0.2970 \mathrm{E}+02$ & $0.1541 \mathrm{E}+02$ & $0.8033 \mathrm{E}-03$ & $0.3005 \mathrm{E}-03$ \\
12 & 75 & 145 & $0.5463 \mathrm{E}+02$ & $0.2826 \mathrm{E}+02$ & $0.7529 \mathrm{E}-03$ & $0.2647 \mathrm{E}-03$
\end{tabular}

Table 2: Results for the function $f_{2}$. The compression ratio $\mu_{\mathrm{cr}}$ and the evaluation compression ratio $\tau$ are computed as shown in the equations (23) and (24), respectively.

state, as follows

$$
\left\{\begin{array}{l}
\frac{\mathrm{d} \rho}{\mathrm{d} t}=\alpha(\bar{\rho}-\rho)-\gamma \rho-\beta(\rho-\bar{\rho}) \rho^{2} \\
\bar{\rho}=1+\frac{1}{2} \sin (5 \omega+8 / 5) \\
\beta=20 \omega,
\end{array}\right.
$$

where $\alpha=1, \gamma=0.01$ and $\omega \sim \mathcal{U}[0,1]$. The original problem [6]is related to the evolution of the surface coverage $\rho$ for a given species. A discontinuous initial solution in the stochastic space is chosen in order to obtain a discontinuous response

$$
\rho(t=0)=\left\{\begin{array}{l}
3 / 4 \text { if } 0.3<\omega<0.7 \\
0 \quad \text { otherwise }
\end{array}\right.
$$

In this case of unsteady problems, the aim is to compute the temporal evolution of the mean and the variance 
following

$$
\begin{aligned}
& \mathcal{E}(t)=\int_{\Xi} f(\xi, t) p(\xi) \mathrm{d} \xi \\
& \operatorname{Var}(t)=\int_{\Xi}(f(\xi, t)-\mathcal{E}(t))^{2} p(\xi) \mathrm{d} \xi .
\end{aligned}
$$

The MC converged solution, $\rho_{\text {ref }}(t)$, is retained as reference for mean and variance solutions. The errors are computed as follows

$$
\left.\operatorname{err}_{\mu^{m}}\right|_{L_{p}}=\left\|\mu^{m}(\rho, t)-\mu^{m}\left(\rho_{\mathrm{ref}}, t\right)\right\|_{L_{p}}=\left(\frac{1}{N_{t}} \sum_{i=1}^{N_{t}}\left|\frac{\mu_{i}^{m}(\rho, t)-\mu_{i}^{m}\left(\rho_{\mathrm{ref}}, t\right)}{\mu_{i}^{m}\left(\rho_{\mathrm{ref}}, t\right)}\right|^{p}\right)^{1 / p},
$$

where $\mu^{m}$ are the statistic moments, i.e. mean $\mathcal{E}$ or variance Var, and $p=1,2$ is referred to the spaces $L_{1}$ or $L_{2}$, respectively. The number of time steps is indicated with $N_{t}$ and the total time of the simulation is equal to $T=2$ where the time step is equal to $\Delta t=0.01\left(N_{t}=200\right)$. Moreover, the $L_{\infty}$ norm has been computed as

$$
\left.\operatorname{err}_{\mu^{m}}\right|_{L_{\infty}}=\left\|\mu^{m}(\rho, t)-\mu^{m}(\bar{\rho}, t)\right\|_{L_{\infty}}=\max _{i}\left|\frac{\mu_{i}^{m}(\rho, t)-\mu_{i}^{m}\left(\rho_{\mathrm{ref}}, t\right)}{\mu_{i}^{m}\left(\rho_{\mathrm{ref}}, t\right)}\right|,
$$

for both mean and variance.

In figures 10, 11 and 12, the results for the errors in $L_{1}, L_{2}$ and $L_{\infty}$ are reported for MC, PC and TE. The number of points $N$ in this case is equal to the total number of points in the grid $\omega-t$, i.e. the product of the number of points in the stochastic space $N_{\xi}$ and the number of time intervals $N_{t}=200$ employed $N=N_{\xi} \times N_{t}$. The reference solution was obtained with a number of stochastic points equal to $N_{\xi}=2.5 \times 10^{6}$. Several set of points were chosen in order to study the convergence of the different methods, in particular $N_{\xi}$ was varied between 10 and 450 with step of 10 for both $\mathrm{MC}$ and $\mathrm{PC}$, while computations performed with $\varepsilon=10^{-2}$ and maximum levels between 3 and 15 . The integration in time was performed by means of an explicit Euler formula.

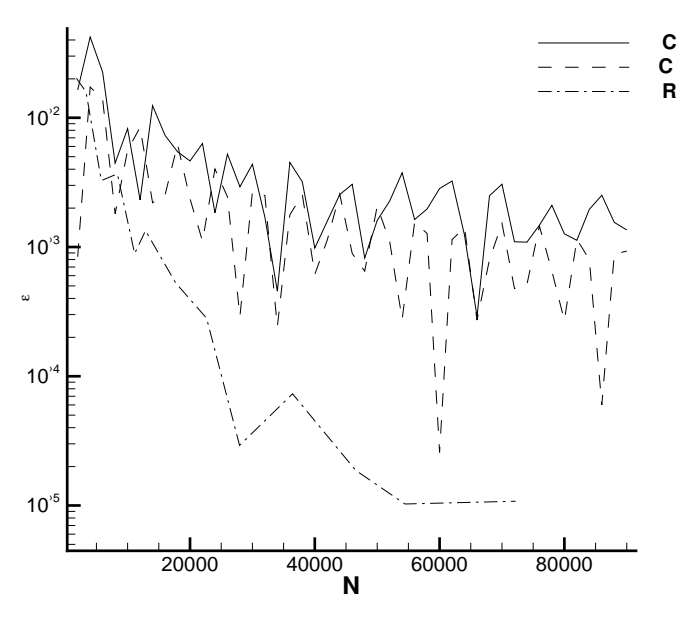

(a)

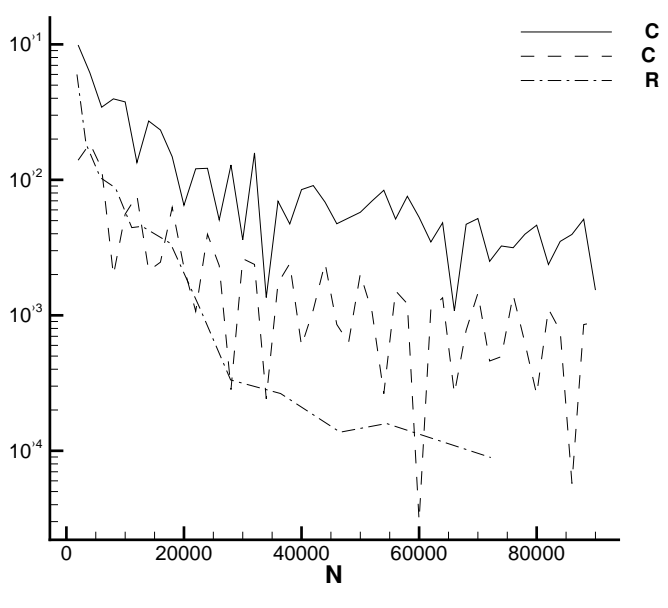

(b)

Figure 10: Errors for the mean (a) and variance (b) in the $L_{1}$ norm for the equation (29) with $m_{L}=1$ and $\varepsilon=10^{-2}$.

The TE algorithm allows reaching a lower level of error at the same number of points. In the case of $L_{\infty}$ norm for the variance, at the same number of points it corresponds an order of magnitude of the error inferior up to three degrees of magnitude. The convergences plots exhibit the smoothest behaviors with respect to MC and PC despite to the presence of two discontinuities in the function $\rho(\xi, t)$.

A plot of the function $\rho(\omega, t)$ is reported in the figure 13(a) displaying only the evaluated points. The same plot is reported in two dimensions (13(b)), where the time evolution of the evaluations is clearer. In both cases, the maximum 


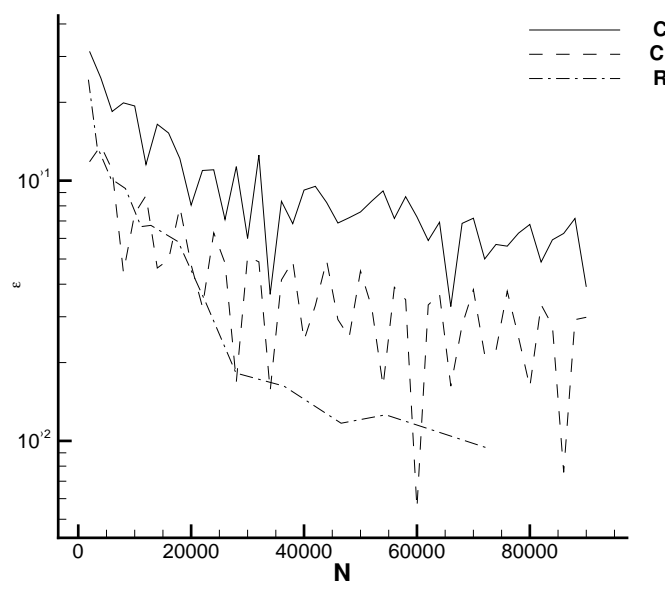

(a)

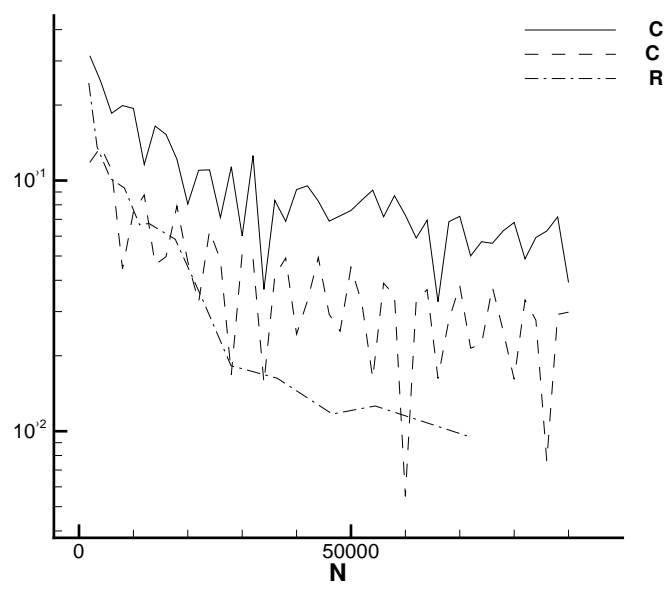

(b)

Figure 11: Errors for the mean (a) and variance (b) in the $L_{2}$ norm for the equation (29) with $m_{L}=1$ and $\varepsilon=10^{-2}$.

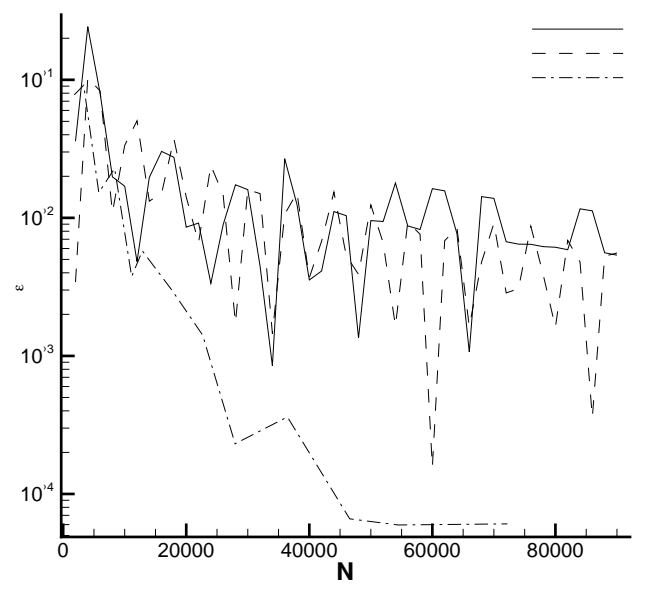

(a)

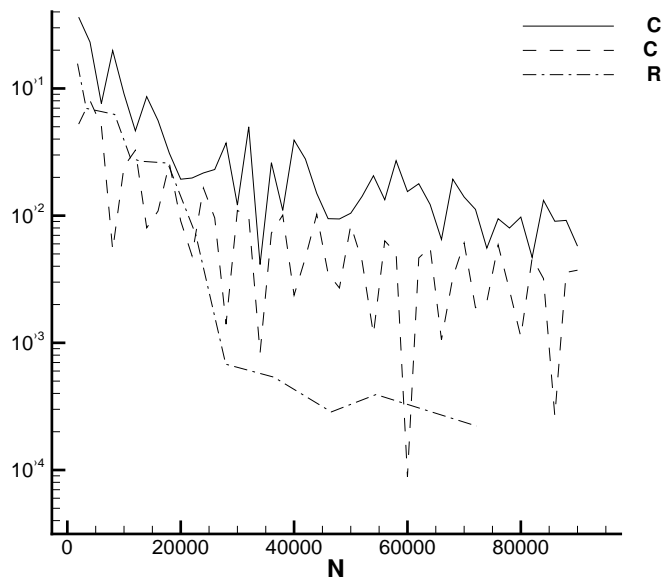

(b)

Figure 12: Errors for the mean (a) and variance (b) in the $L_{\infty}$ norm for the equation (29) with $m_{L}=1$ and $\varepsilon=10^{-2}$.

level was fixed to 8 with $m_{l}=1$ and $\varepsilon=10^{-2}$. It is possible to recognize the two discontinuities located in $\omega=0.3$ and $\omega=0.7$ where more points are collocated by the TE algorithm. One discontinuity at $\omega=0.3$, disappears before the second one. At $t=1$, the TE algorithm is capable to respect the accuracy requirements without collocating more points in these $\omega$ stations because the function reaches a smoother state.

The behavior shown in the figure 13 displays the refinement/derefinement capability of the algorithm to move and collocate points in the stochastic space with respect to the time evolution. For example, in figure 14, the evolution of the evaluated points in time is reported employing a maximum level equal to 8 , corresponding to a total number of evaluations equal to 257, with $m_{l}=1$ and $\varepsilon=10^{-2}$. It is evident that in proximity of $t=1$, when both the discontinuity disappear, the number of points changes abruptly. However, after $t=1$, the development of a region characterized by higher gradient (see figure 13(a)), requires a greater number of points as it is evident in figure 14 . 


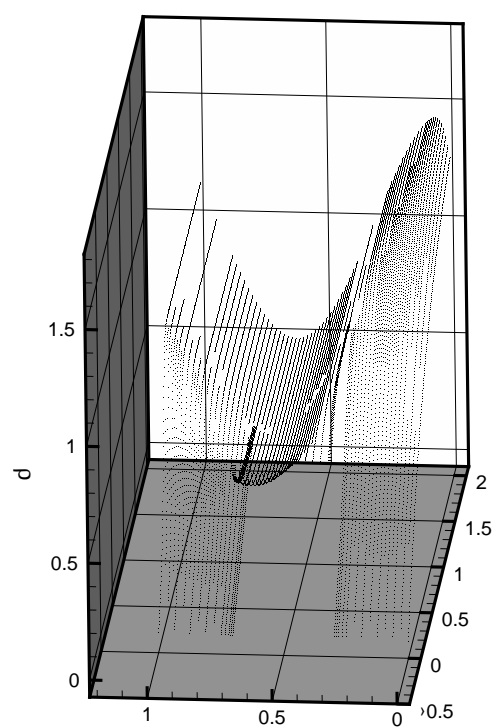

$\omega$

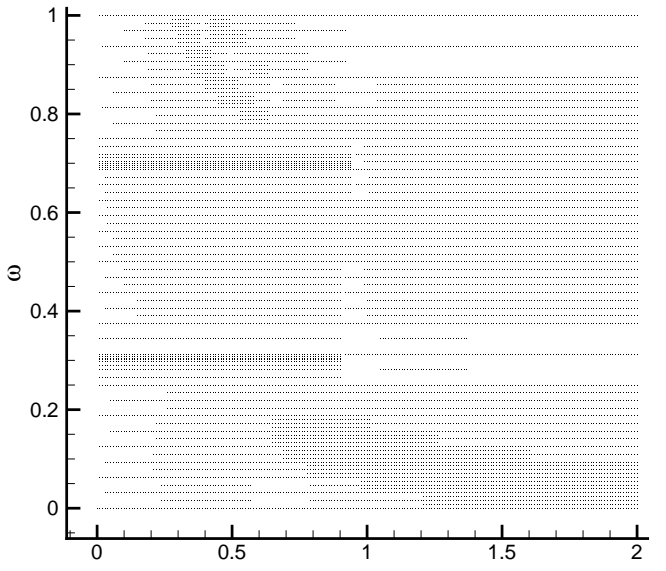

(b)

Figure 13: Patterns of the evaluations of $\rho(\omega, t)$ in the space $\omega-t$ in a 3D ((a)) and 2D ((b)) frames.

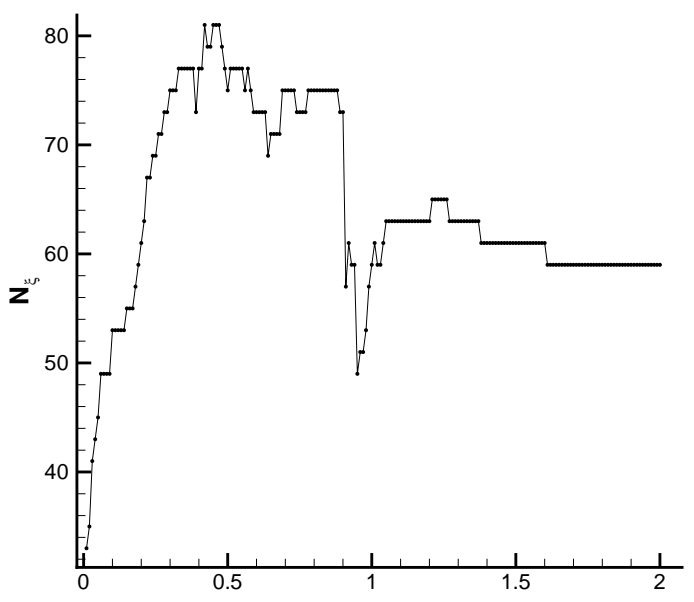

Figure 14: Time evolutions of the evaluations.

The pattern of the evaluated points and the activated ones, with respect to the time evolution, is reported in figure 15. The parameters are the same of the previous cases, i.e. $m_{l}=1, m_{\max }=8$ and $\varepsilon=10^{-2}$. Obviously, due to the discontinuities, the TE algorithm generates all the levels up to $m_{\max }$.

Now, let us apply the TE algorithm to a vectorial case, i.e. the stiff Kraichnan-Orszag problem in one stochastic dimension. 


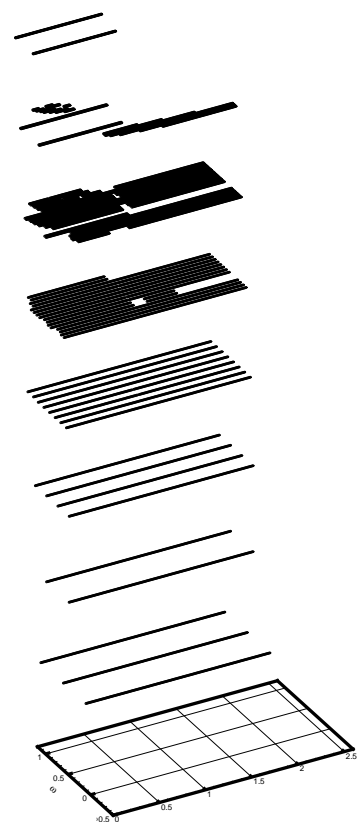

(a)

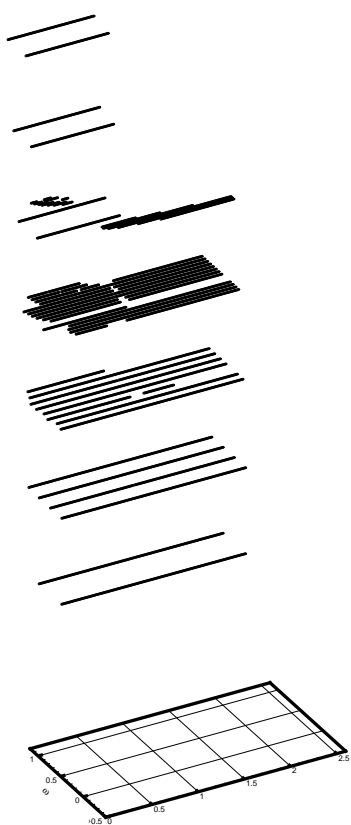

(b)

Figure 15: Pattern of the computed (a) and activated (b) points in time for the application of the TE algorithm on the equation (29).

\subsection{A time dependent vectorial ODE: the Kraichnan-Orszag problem}

The so-called Kraichnan-Orszag problem was proposed in 1967 [28] by Orszag as a three mode problem which can be seen as an inviscid turbulence model given by a set of ordinary differential equations. Actually this differential model is considered a stiff problem for the solution of stochastic problems, due to its high non-linearity. The original system, rotated by $\pi / 4$ around the axis $y_{3}$ can be written following [4] as

$$
\left\{\begin{array}{l}
\frac{\mathrm{d} y_{1}}{\mathrm{~d} t}=y_{1} y_{3} \\
\frac{\mathrm{d} y_{2}}{\mathrm{~d} t}=-y_{2} y_{3} \\
\frac{\mathrm{d} y_{3}}{\mathrm{~d} t}=-y_{1}^{2}+y_{2}^{2} .
\end{array}\right.
$$

To formulate the 1D problem, the equation (34) must be correlated with the following (uncertain) initial condition $\mathbf{y}(t=0)=(1,0.1 \xi, 0)^{\mathrm{T}}$, in which the parameter $\xi$ is uniformly distributed between -1 and $1, \xi=2 \omega-1$, where $\omega \sim \mathcal{U}[0,1]$. The numerical integration scheme used in this work is the classical Runge-Kutta (RK4) with a time step of 0.05 , chosen after a convergence study not reported here for brevity.

The pattern of the solution is reported (in the state space) in figure 16, where the TE algorithm performed with a maximal level equal to 8 starting with $m_{L}=4$ and a threshold $\varepsilon=10^{-1}$.

The evolution of the three variables in time is reported in figure 17, where the TE algorithm is applied with $m_{\max }=8, m_{L}=4$ and $\varepsilon=10^{-1}$. Remark that this set of parameter is chosen only to show qualitatively the pattern of points even if better results could be obtained using an higher maximal level $m_{\max }$.

Observing figure 17 , it is evident that discontinuities occur crossing the plane at $\xi=0$. Moreover, the variables distributions are even with respect to the axis $\xi=0$ for the variables $y_{1}$ and $y_{3}$ while is odd for the variable $y_{2}$. This behavior produces a zero mean for the variable $y_{2}$.

The global behavior of the mean and variance in time for the three variables has been reported in figure 18. The number of simulations used by the TE algorithm with $\varepsilon=10^{-1}, m_{L}=4$ and $m_{\max }=8$ is equal to $N_{\xi}=89$. In order to assess the results obtained for the mean and variance of the three variables, the PC and MC methods are applied 


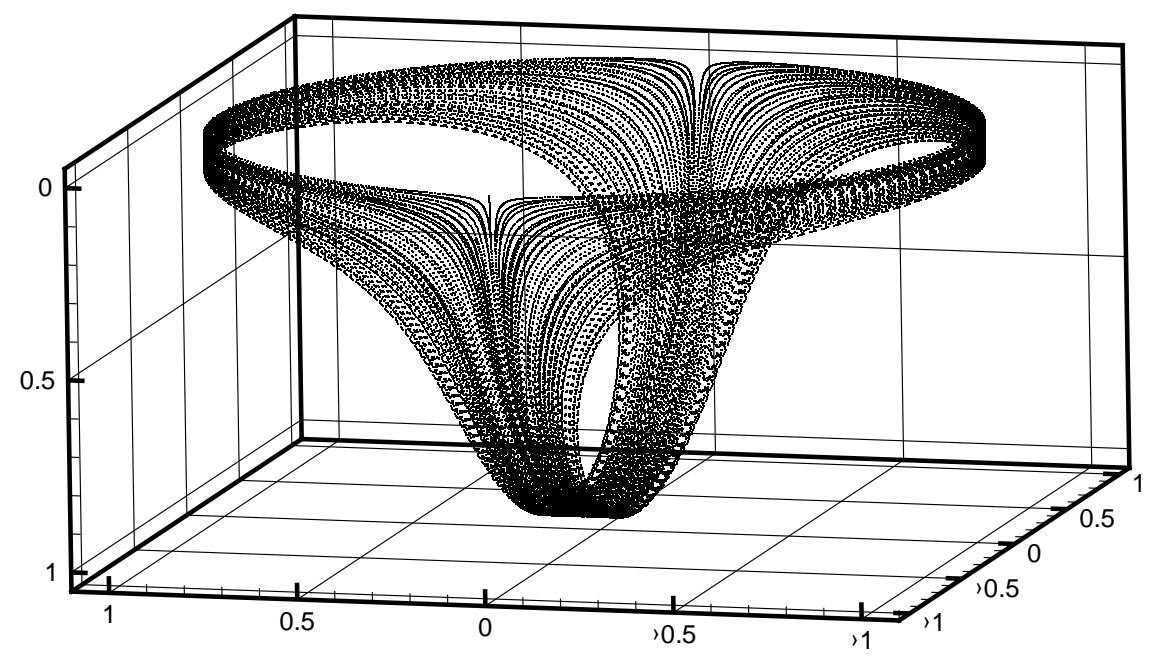

Figure 16: Pattern of the computed points for the solution of the equation (29).

by using the same number of points. The statistics for the three variables are reported in the figure 18 , where the reference solution obtained with $N_{\xi}=20 \times 10^{6}$ is also reported. Concerning the variable $y_{2}$, the MC is not capable to predict a zero-value at the machine accuracy even in the case of the reference solution $\left(N_{\xi}=20 \times 10^{6}\right)$; in particular, the solution for the reference case is of the order of $10^{-8}$, while for the case $N_{\xi}=89$ is of the order of $10^{-3}$. For this reason, only the PC and MR results are reported in figure 18(c). Remark that only the TE algorithm is capable to compute zero-values, accurate at the machine accuracy.

For this vectorial ODE case, the error in norms are computed for the three variables according to the definitions (32) and (33). We remark that normalizing the norms for the mean is not easy because the reference value could be equal, or almost equal to zero. In particular $\mathcal{E}\left(y_{2}\right)$ should be exactly zero, while $\mathcal{E}\left(y_{3}\right)$ cross periodically zero. Moreover, the variances for all the variables start from zero. For these reasons, we report in figure 19 the results for the mean $\mathcal{E}\left(y_{1}\right)$ and all the variances, but in this last case the norms are computed in a bound time interval $[8,30]($ as chosen by [4]). Note that the value $N_{t}$ considered in the equations (8) is, nevertheless, equivalent to the overall time interval $[0,30]$.

In the figure 19, the errors for the mean of the variable $y_{1}$ are reported measured in the $L_{1}, L_{2}$ and $L_{\infty}$ spaces.

In the figures 20,21 and 22, the error norms of the variance in the time interval [8,30] (and in the $L_{1}, L_{2}$ and $L_{\infty}$ spaces) are reported for the variables $y_{1}, y_{2}$ and $y_{3}$, respectively.

The points distribution, where the function is evaluated, are reported in figure 23. Even in this vectorial case, the TE algorithm displays good derefinement properties. Therefore, the points are distributed in the regions of higher gradients, or discontinuity and locally, between successive time levels (see figure 23(b)).

All the results of this section were obtained for stochastic parameters characterized by an uniform distribution. A 


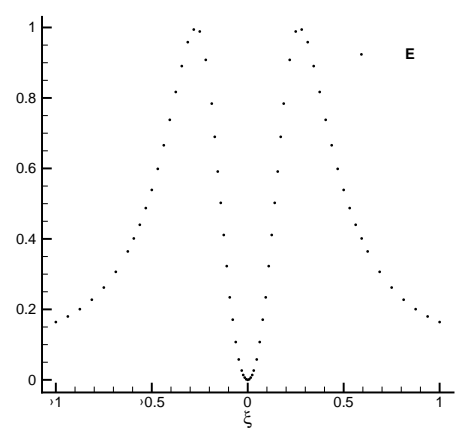

(a)

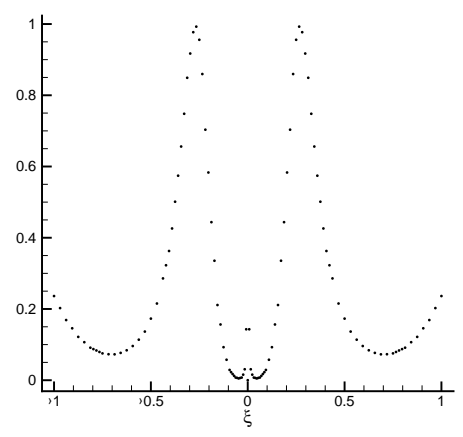

(d)

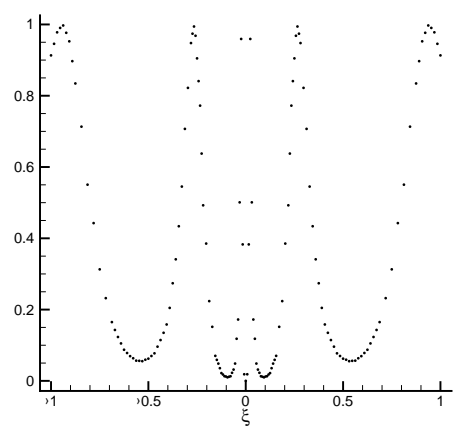

(g)

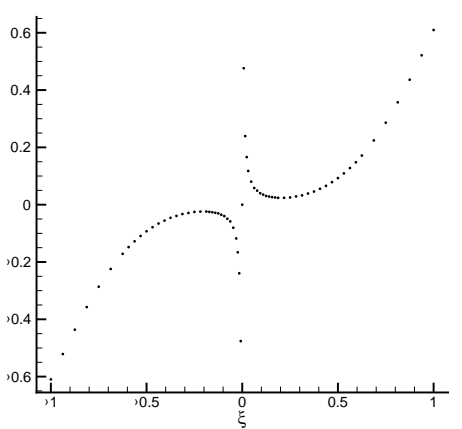

(b)

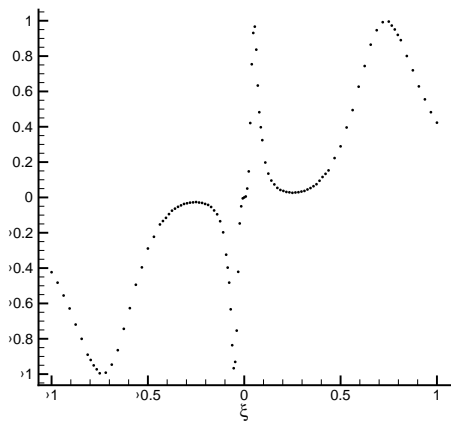

(e)

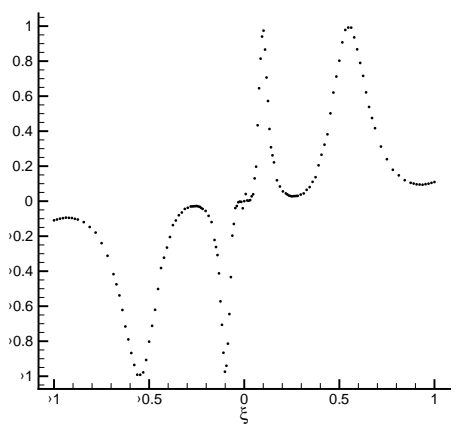

(h)

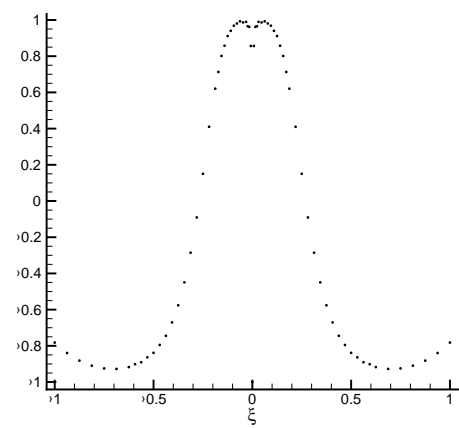

(c)

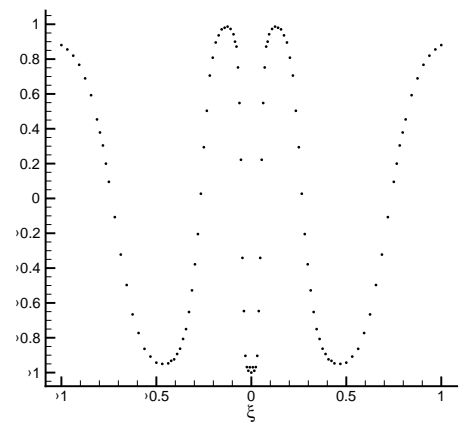

(f)

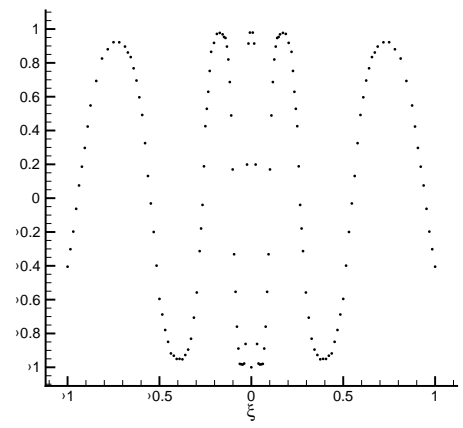

(i)

Figure 17: Time evolution of the variables $y_{1}$ (left), $y_{2}$ (middle) and $y_{3}$ (right) at different time steps: $t=10((\mathrm{a}),(\mathrm{b})$ and (c)), $t=20((\mathrm{~d}),(\mathrm{e})$ and (f)) and $t=30((\mathrm{~g}),(\mathrm{h})$ and (i)). $x$ interpolated points and $\cdot$ evaluated ones.

more challenging problem, where a time varying and discontinuous pdf is considered, is reported in the section $\S 6.5$.

\subsection{Some remarks on the long-time integration problem}

The aim of this section is to show how the TE algorithm can tackle the long-time integration problem. For this reason, the 1D Kraichnan-Orszag problem, with uniform probability density function for the random parameter $\xi$, is been solved for a time up to 500. Remark that intrusive techniques like the PC, as demonstrated in [4], fails to perform this kind of computation, even for smaller time. The proposed approach can lead to the correct evolution in time of 
the statistics only if the error cumulated at each time step remains bounded. As described in the section 4 , the time integration procedure to advance in time allows moving a compressed solution with a prescribed accuracy requirement. These accuracy requirements allow bounding the error that can accumulate during several time integrations: the final results is a strong decreasing of the number of total evaluations employed to reproduce the exact solution even for long time.

As in the previous case, the MC method, with $N_{\xi}=2 \times 10^{6}$ points, is used as reference solution.

In figure 24, the time evolution of the variance for the three variables $y_{1}, y_{2}$ and $y_{3}$ is reported for the last part of the simulation, i.e. $t \in[450,500]$. The results in the figure 24 are obtained employing the TE algorithm with $m_{L}=4$, a maximum level with $m_{\max }=12$ and a threshold $\varepsilon=10^{-3}$.

In the figure 25, the errors for the mean of the variable $y_{1}$ are reported measured in the $L_{1}, L_{2}$ and $L_{\infty}$ spaces.

The error norms of the variances computed in the time interval $[8,500]$ (in the $L_{1}, L_{2}$ and $L_{\infty}$ spaces) are reported for the variables $y_{1}, y_{2}$ and $y_{3}$, in figures 26,27 and 28 , respectively.

Remark that, in the case of long-time integration, the advantages of the application of the TE algorithm appear very poor with respect to the short-time case reported in section 6.3 or the unsteady pdf reported in section 6.5 . Nevertheless despite to the very high complexity of the solution (see figure 29), all the norms of the errors computed for the three variables are smaller in the case of the application of the TE algorithm.

In figures 25, 26, 27 and 28, the TE algorithm is applied with different thresholds in order to display the different convergence properties with a fixed coarsest level correspondent to $m_{L}=4$ and a maximal level equal to $m_{\max }$ between 6 and 11. According to the theoretical prediction, an higher threshold correspond to an higher error. However, we remark that, in the long time case reported in this section, a lower threshold is needed in order to obtain better results with respect to a PC method. Instead, for the other numerical experiences reported in sections 6.3 and 6.4, an higher threshold $\left(\varepsilon=10^{-1}\right)$ is sufficient to ensure the best trade-off between a reduced number of points and the lowest error. In general, we can say that long-time highly non-linear problems require a lower threshold due to control the interpolation error cumulated during the time-integration.

The evolution of the number of evaluations in time, for the TE algorithm, is reported in figure 30, where the refinement/derefinement properties of the algorithm can lead to a reduced number of simulations with respect to the finest resolution level in the initial stage of the computation (for $t$ less than about 120). While, for increasing $t$, an higher number of simulation is required with no possibility to reduce, even locally, the number of evaluations due to the high non-linearity of the solution (see figure 29 in which all the points consist in evaluations). However, the reduction of the number of evaluations in the first part of the computation is sufficient to ensure a good reduction of the overall number of computations.

\section{Some additional remarks on the computational cost}

In this case, we report explicitly the computational cost associated to TE algorithm in terms of time. In the table 3, the number of points evaluated $\left(N_{\text {eval }}\right)$, the evaluation compression ratio (see equation (24)) and a measure of the saved time $\Delta t \%$ by using the TE algorithm are reported. If we indicate with $T_{F U L L}$ the time employed without compression for a certain level $m_{\max }$ and $t_{\varepsilon}$ the time needed by the TE algorithm for a certain threshold $\varepsilon$ (obviously with the same maximum level), then $\Delta t \%$ is computed as

$$
\Delta t \%=\frac{T_{F U L L}-t_{\varepsilon}}{T_{F U L L}} \times 100
$$

All the data are relative to the simulations performed on a personal laptop embedded with Intel (R) Core (TM) 2 Duo CPU P9700 @ $2.80 \mathrm{GHz}$ and 4GB of RAM. The evolution of the saved time using the TE algorithm is reported, as function of the maximum level $m_{\max }$ for different threshold in the figure 31 . 


\begin{tabular}{c|c|c|c|c}
$m_{\max }$ & $N_{\text {eval }}$ & $t_{\varepsilon}[\mathrm{s}]$ & $\tau$ & $\Delta t \%$ \\
\hline \hline \multicolumn{5}{c}{$\varepsilon=10^{-} 1$} \\
\hline $6.0000 \mathrm{E}+00$ & $6.3788 \mathrm{E}+05$ & $4.0400 \mathrm{E}+00$ & $1.0190 \mathrm{E}+00$ & $4.3866 \mathrm{E}-01$ \\
$8.0000 \mathrm{E}+00$ & $2.3891 \mathrm{E}+06$ & $1.1664 \mathrm{E}+01$ & $1.0757 \mathrm{E}+00$ & $5.6885 \mathrm{E}+00$ \\
$1.0000 \mathrm{E}+01$ & $8.3288 \mathrm{E}+06$ & $3.7696 \mathrm{E}+01$ & $1.2307 \mathrm{E}+00$ & $1.9874 \mathrm{E}+01$ \\
$1.2000 \mathrm{E}+01$ & $2.2681 \mathrm{E}+07$ & $1.0149 \mathrm{E}+02$ & $1.8064 \mathrm{E}+00$ & $4.5052 \mathrm{E}+01$ \\
\hline $6.0000 \mathrm{E}+00$ & $6.4681 \mathrm{E}+05$ & $4.0569 \mathrm{E}+00$ & $1.0049 \mathrm{E}+00$ & $2.2180 \mathrm{E}-02$ \\
$8.0000 \mathrm{E}+00$ & $2.5113 \mathrm{E}+06$ & $1.2081 \mathrm{E}+01$ & $1.0234 \mathrm{E}+00$ & $2.3110 \mathrm{E}+00$ \\
$1.0000 \mathrm{E}+01$ & $9.5236 \mathrm{E}+06$ & $4.4494 \mathrm{E}+01$ & $1.0763 \mathrm{E}+00$ & $5.4243 \mathrm{E}+00$ \\
$1.2000 \mathrm{E}+01$ & $3.3905 \mathrm{E}+07$ & $1.5353 \mathrm{E}+02$ & $1.2084 \mathrm{E}+00$ & $2.2288 \mathrm{E}+01$ \\
\multicolumn{5}{c}{$\varepsilon=10^{-} 3$} \\
\hline $6.0000 \mathrm{E}+00$ & $6.4848 \mathrm{E}+05$ & $4.0533 \mathrm{E}+00$ & $1.0023 \mathrm{E}+00$ & $1.1016 \mathrm{E}-01$ \\
$8.0000 \mathrm{E}+00$ & $2.5484 \mathrm{E}+06$ & $1.2087 \mathrm{E}+01$ & $1.0085 \mathrm{E}+00$ & $2.2672 \mathrm{E}+00$ \\
$1.0000 \mathrm{E}+01$ & $9.9572 \mathrm{E}+06$ & $4.6031 \mathrm{E}+01$ & $1.0294 \mathrm{E}+00$ & $2.1558 \mathrm{E}+00$ \\
$1.2000 \mathrm{E}+01$ & $3.7604 \mathrm{E}+07$ & $1.7134 \mathrm{E}+02$ & $1.0895 \mathrm{E}+00$ & $7.2291 \mathrm{E}+00$
\end{tabular}

Table 3: Time elapsed for the Kraichnan-Orszag problem (with final time $T=500$ ) with the performances of the TE algorithm with different thresholds $\varepsilon$. 


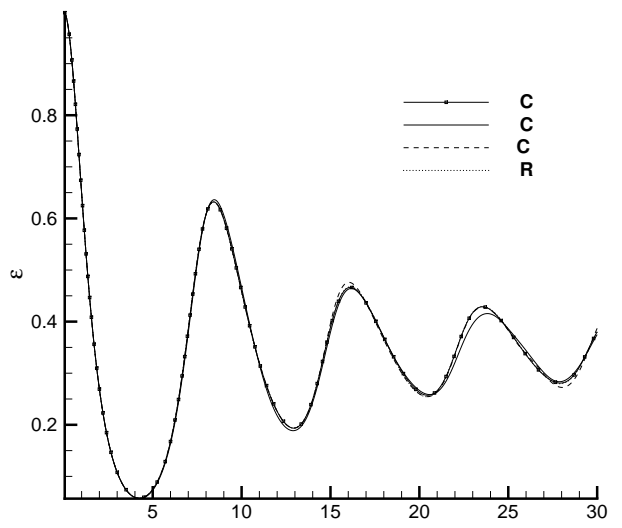

(a)

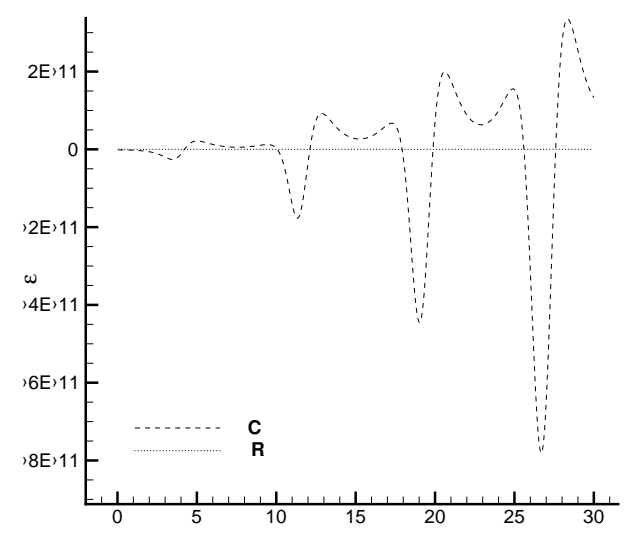

(c)

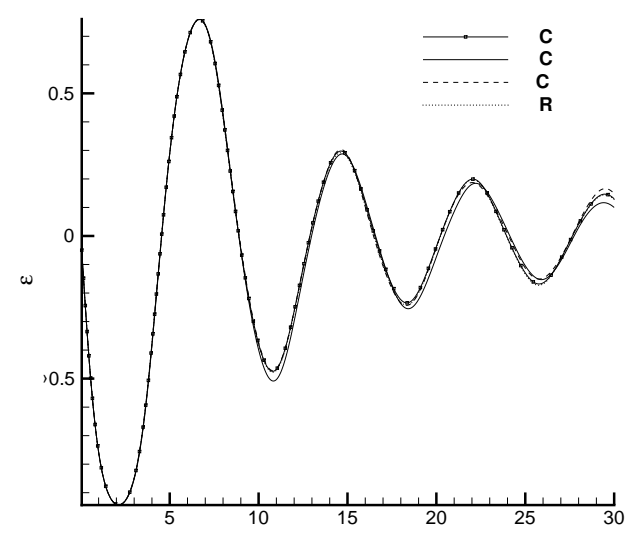

(e)

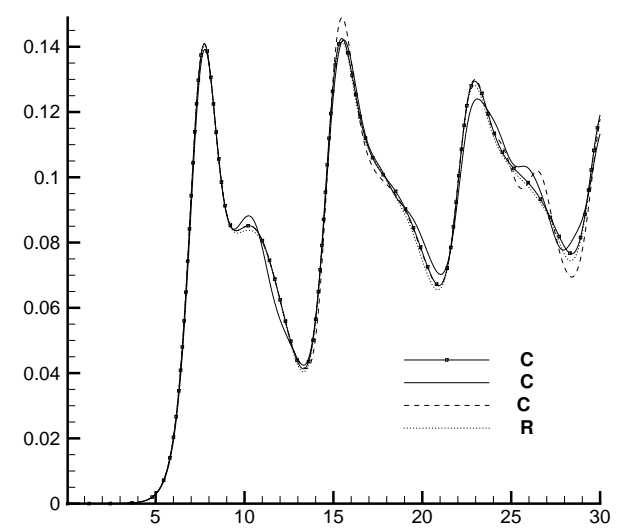

(b)

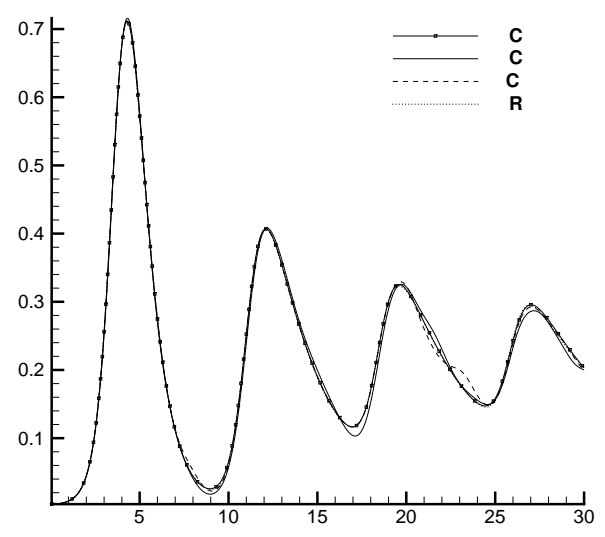

(d)

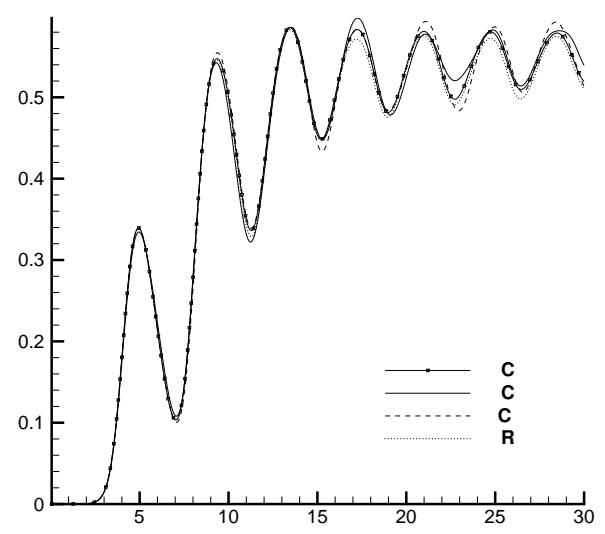

(f)

Figure 18: Time evolution of the mean (left) and variance (right) for the three variables $y_{1}$ (up), $y_{2}$ (middle) and $y_{3}$ (bottom). $\operatorname{RR} n^{\circ} 7967$ 


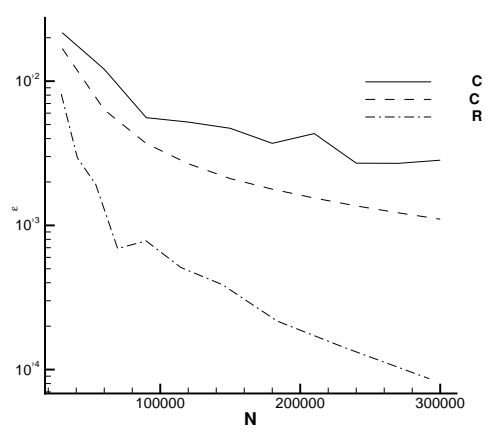

(a)

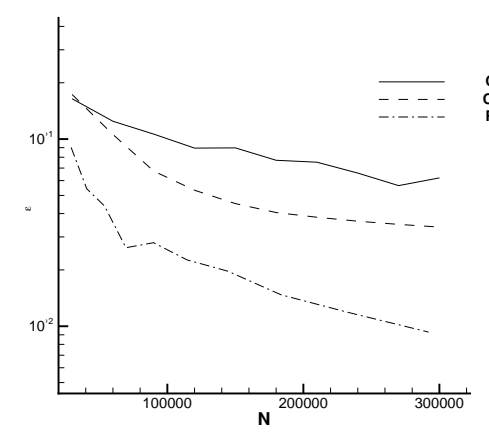

(b)

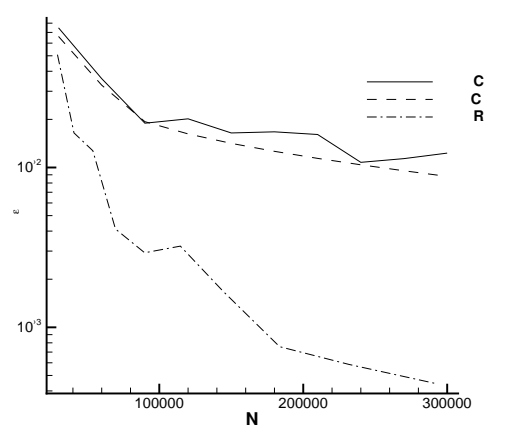

(c)

Figure 19: Error norms of the mean of the variable $y_{1}$ for the 1D Kraichnan-Orszag problem with uniform pdf in the $L_{1}$ (a), $L_{2}$ (b) and $L_{\infty}$ (c) spaces.

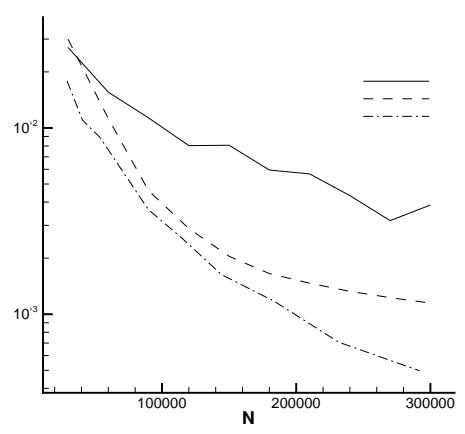

(a)

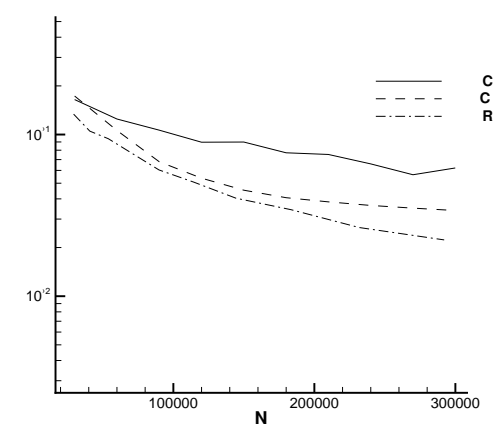

(b)

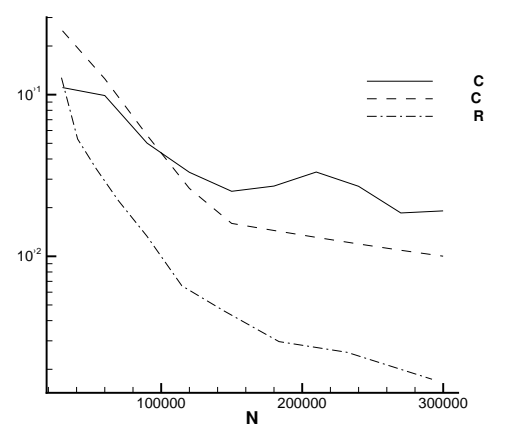

(c)

Figure 20: Error norms of the variance of the variable $y_{1}$ for the 1D Kraichnan-Orszag problem with uniform pdf in the $L_{1}$ (a), $L_{2}$ (b) and $L_{\infty}$ (c) spaces.

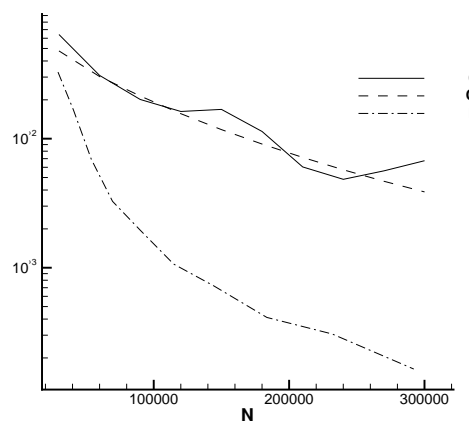

(a)

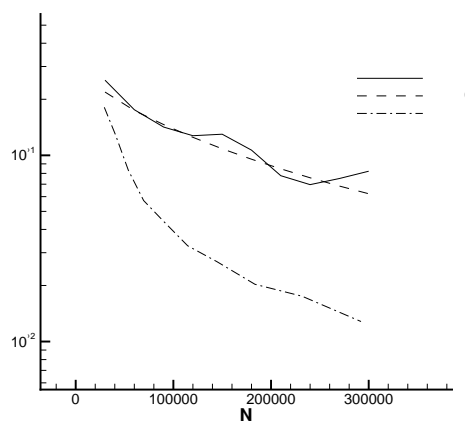

(b)

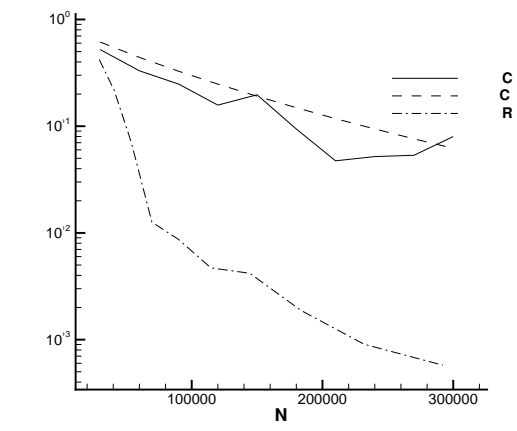

(c)

Figure 21: Error norms of the mean of the variable $y_{2}$ for the 1D Kraichnan-Orszag problem with uniform pdf in the $L_{1}\left(\right.$ a), $L_{2}$ (b) and $L_{\infty}$ (c) spaces.

$R R n^{\circ} 7967$ 


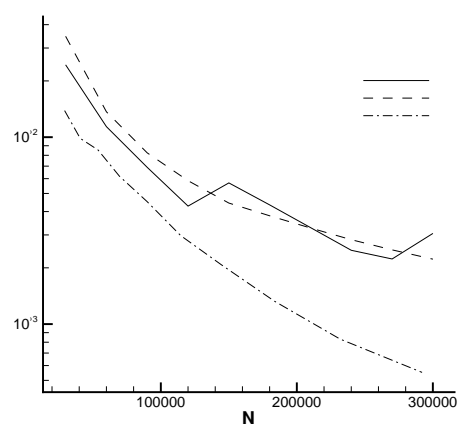

(a)

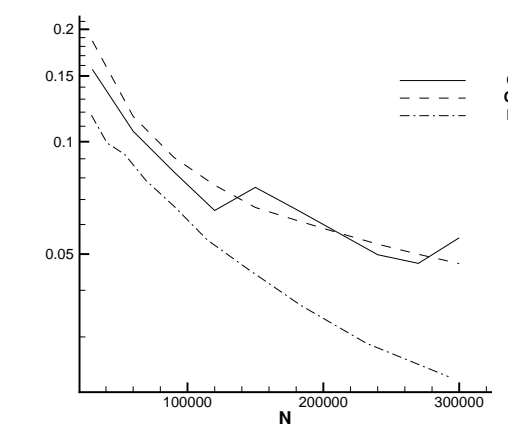

(b)

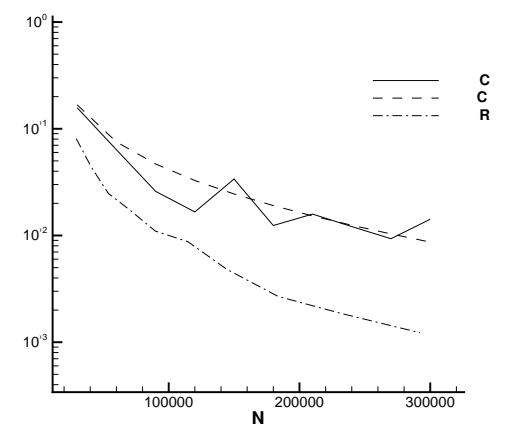

(c)

Figure 22: Error norms of the mean of the variable $y_{1}$ for the 1D Kraichnan-Orszag problem with uniform pdf in the $L_{1}$ (a), $L_{2}$ (b) and $L_{\infty}$ (c) spaces.

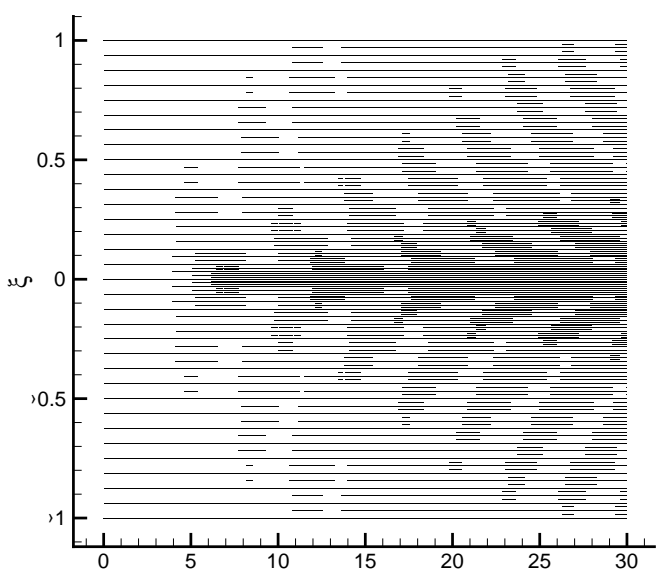

(a)

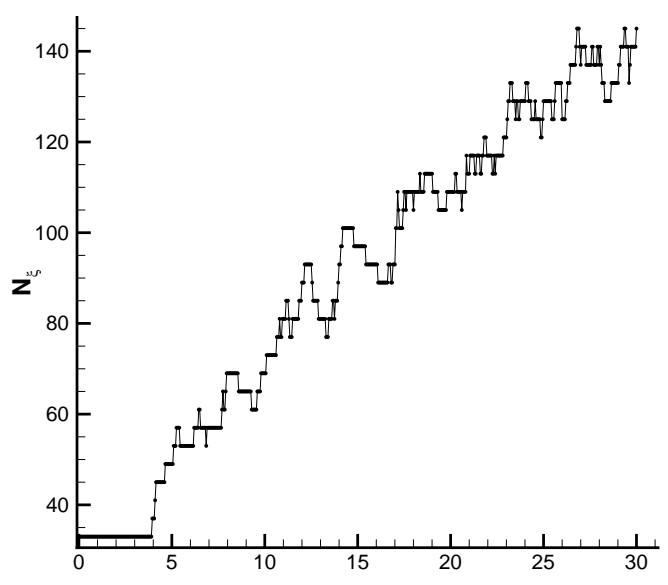

(b)

Figure 23: Patterns of the evaluations in the space $t-\omega((\mathrm{a}))$ and number of points in the stochastic space $N_{\xi}$ employed by the TE algorithm with $\left.m_{\max }=8(\mathrm{~b})\right)$. 


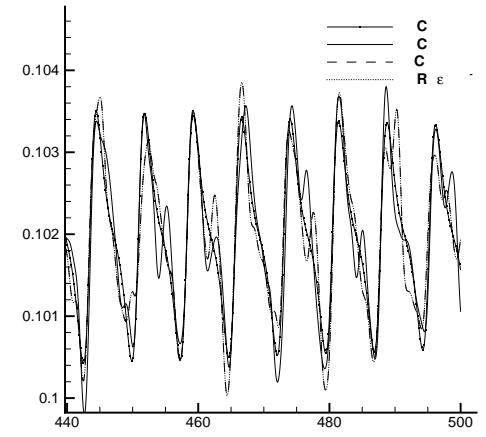

(a)

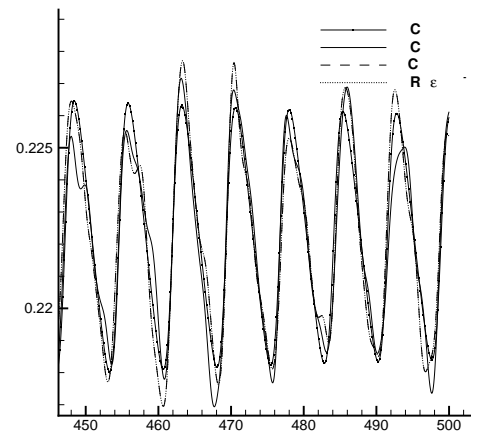

(b)

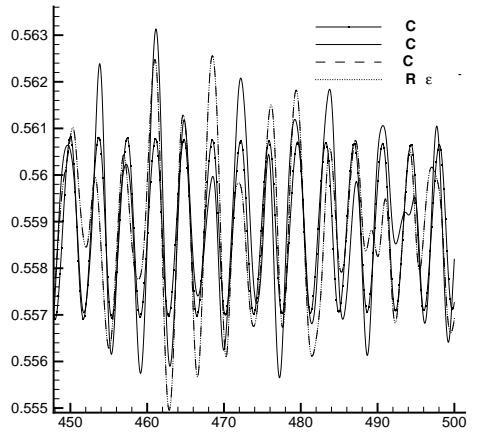

(c)

Figure 24: Comparison of the time evolution for the variance of the variables $y_{1}$ (a), $y_{2}$ (b) and $y_{3}$ (c) in the Kraichnan-Orszag problem with uniform distribution $(T=500)$. The last time steps are computed with the Monte Carlo, Polynomial Chaos and TE algorithm, $\varepsilon=10^{-3}, m_{L}=4$ and $m_{\max }=12\left(N_{\xi}=3760\right)$, and compared to the Monte Carlo reference solution $N_{\xi}=20 \times 10^{6}$.

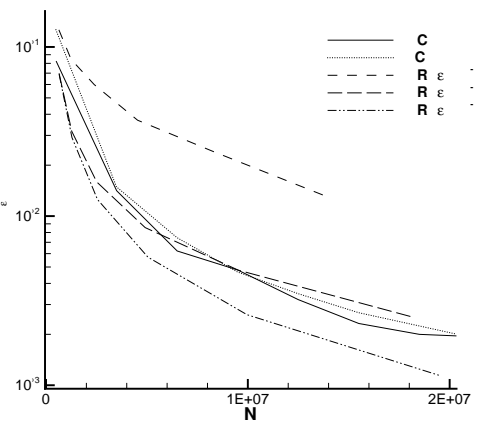

(a)

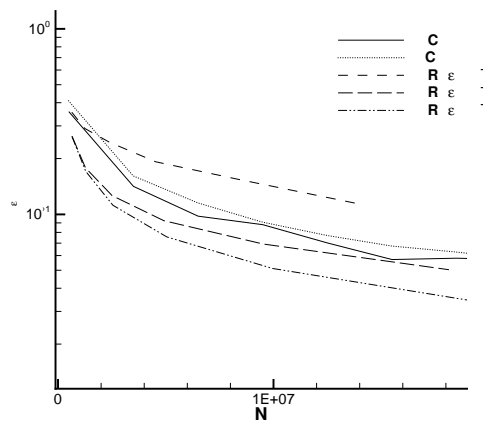

(b)

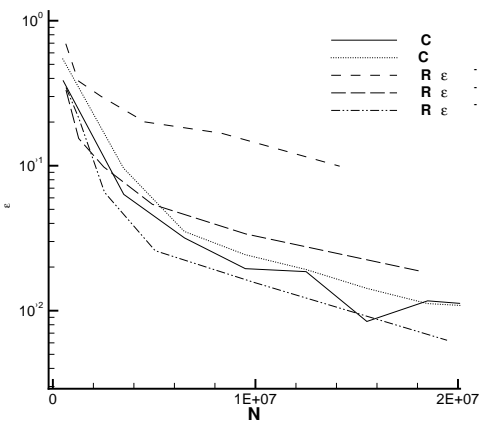

(c)

Figure 25: Error norms for the mean of the variable $y_{1}$ of the 1D Kraichnan-Orszag (for long time $T=500$ ) problem with uniform pdf in the $L_{1}$ (a), $L_{2}\left(\right.$ b) and $L_{\infty}$ (c) spaces.

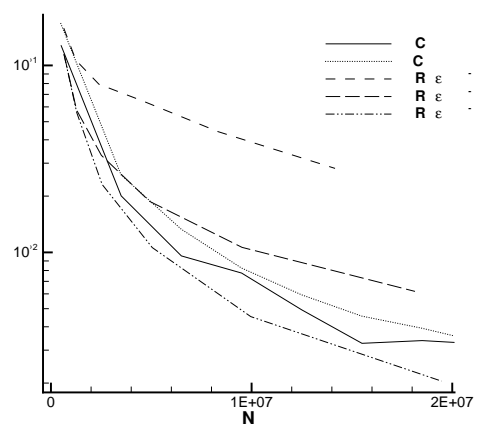

(a)

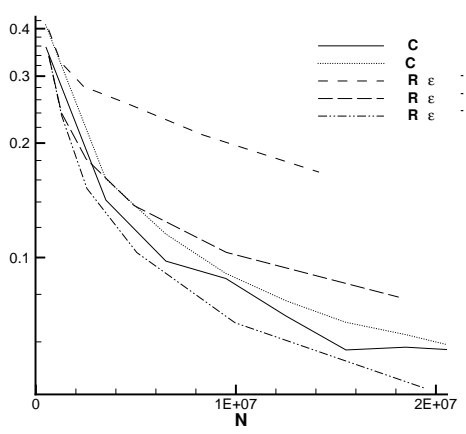

(b)

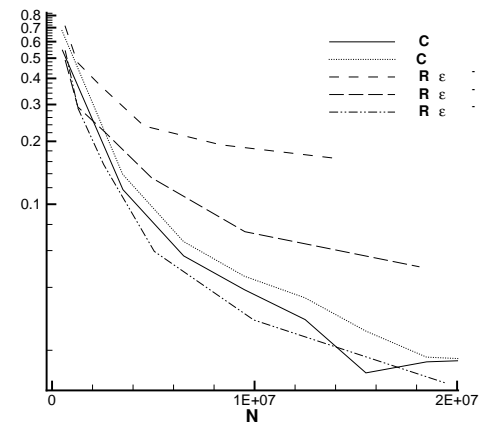

(c)

Figure 26: Error norms of the variance of the variable $y_{1}$ for the 1D Kraichnan-Orszag (for long time $T=500$ ) problem with uniform pdf in the $L_{1}$ (a), $L_{2}$ (b) and $L_{\infty}$ (c) spaces.

$\mathrm{RR} \mathrm{n}^{\circ} 7967$ 


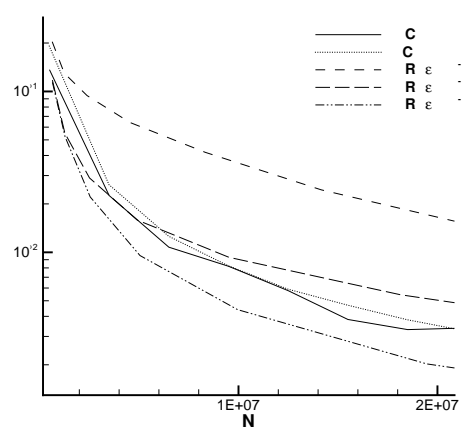

(a)

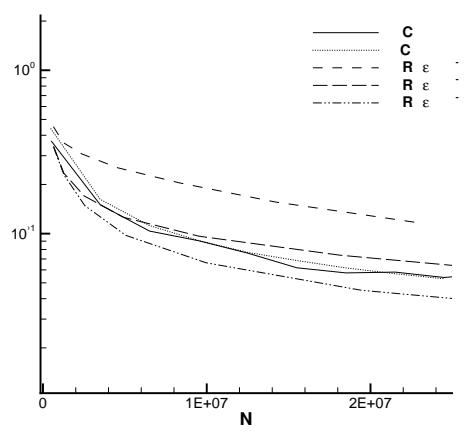

(b)

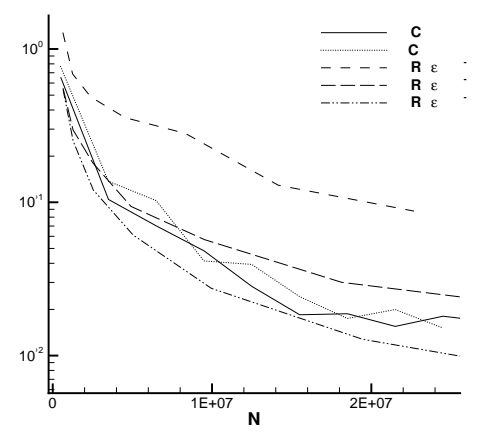

(c)

Figure 27: Error norms of the mean of the variable $y_{2}$ for the 1D Kraichnan-Orszag (for long time $T=500$ ) problem with uniform pdf in the $L_{1}$ (a), $L_{2}\left(\right.$ b) and $L_{\infty}$ (c) spaces.

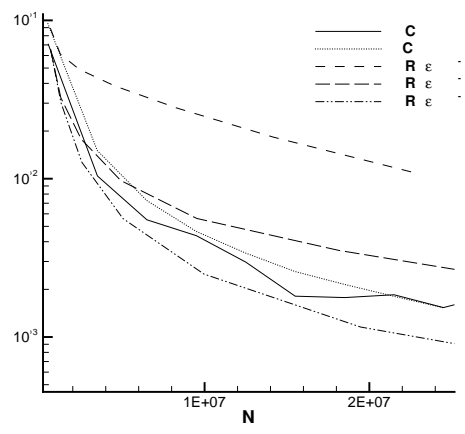

(a)

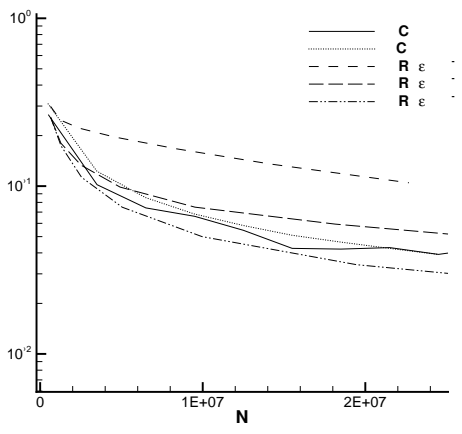

(b)

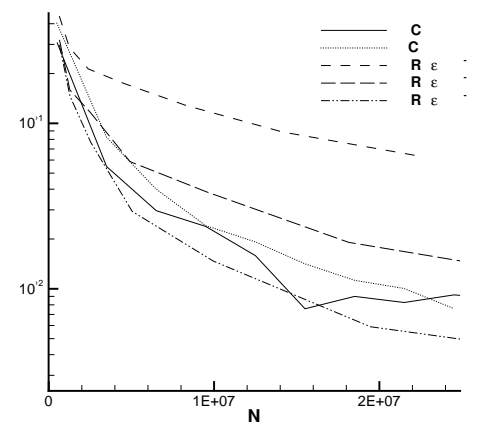

(c)

Figure 28: Error norms of the mean of the variable $y_{3}$ for the 1D Kraichnan-Orszag (for long time $T=500$ ) problem with uniform pdf in the $L_{1}$ (a), $L_{2}$ (b) and $L_{\infty}$ (c) spaces. 


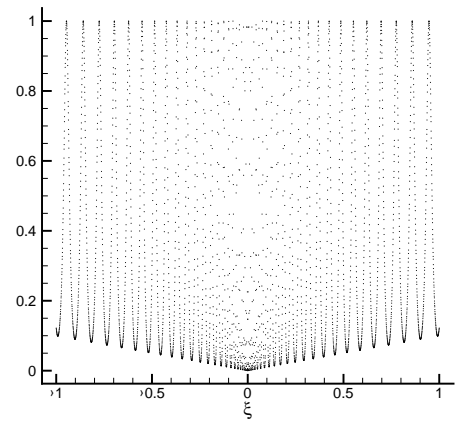

(a)

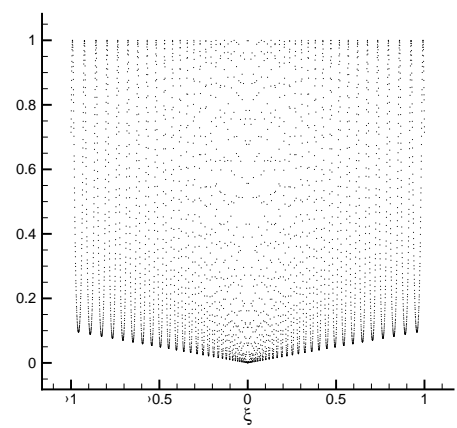

(d)

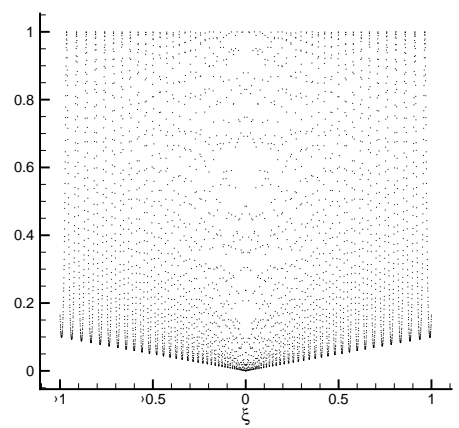

(g)

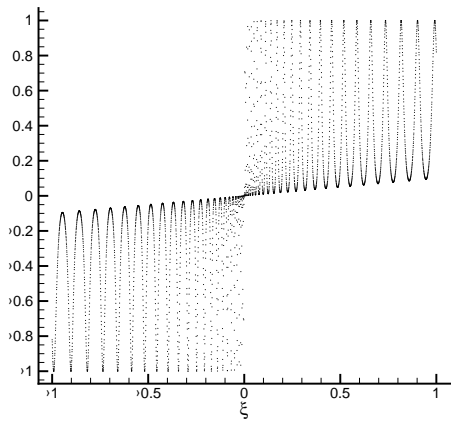

(b)

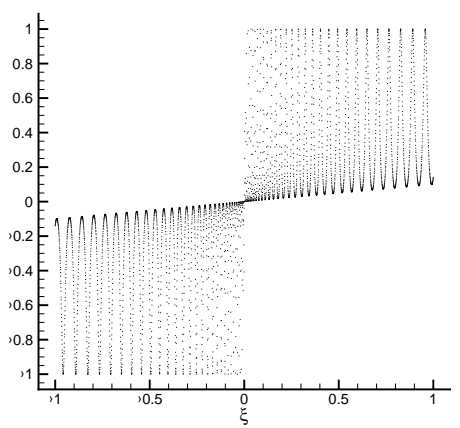

(e)

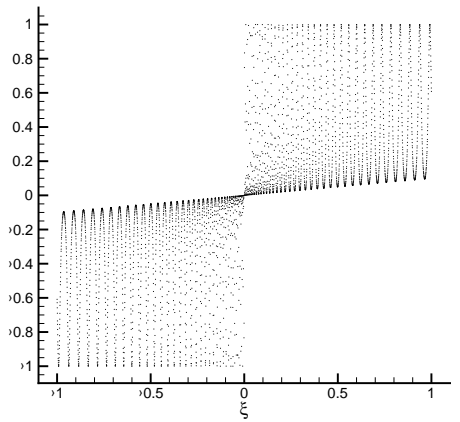

(h)

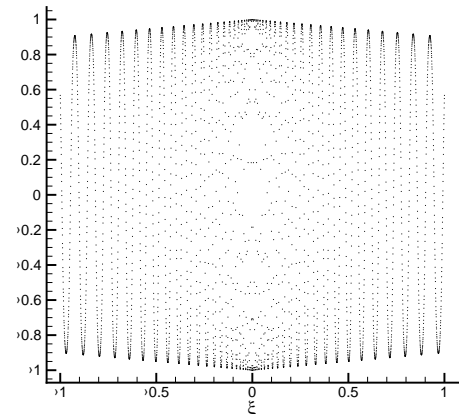

(c)

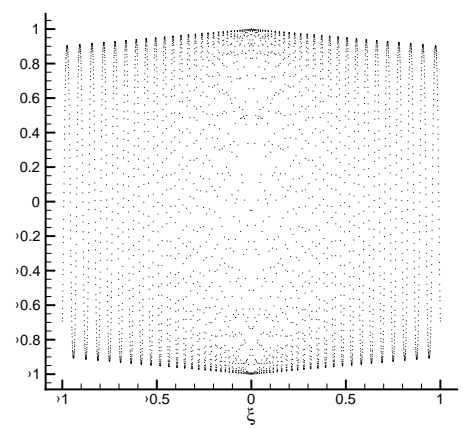

(f)

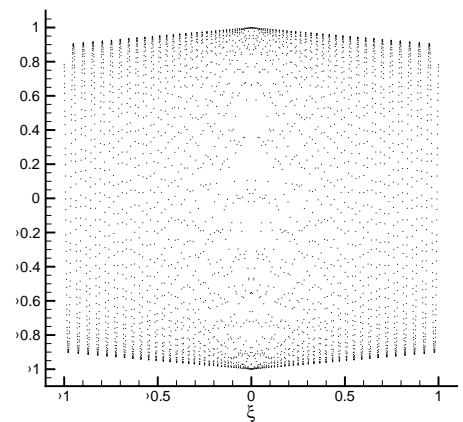

(i)

Figure 29: Time evolution of the variables $y_{1}$ (left), $y_{2}$ (middle) and $y_{3}$ (right) at different time steps: $t=300$ ((a), (b) and (c)), $t=400$ ((d), (e) and (f)) and $t=500((\mathrm{~g}),(\mathrm{h})$ and (i)). 


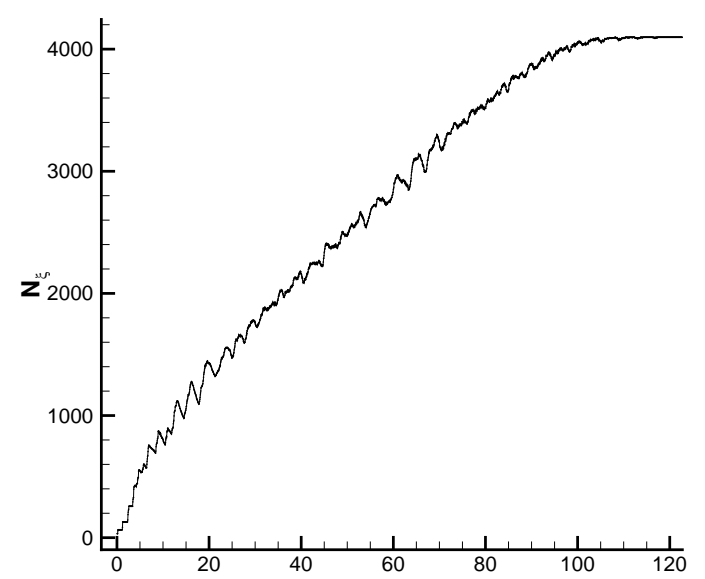

Figure 30: Number of points in the stochastic space $N_{\xi}$ employed by the TE algorithm with $m_{\max }=12$.

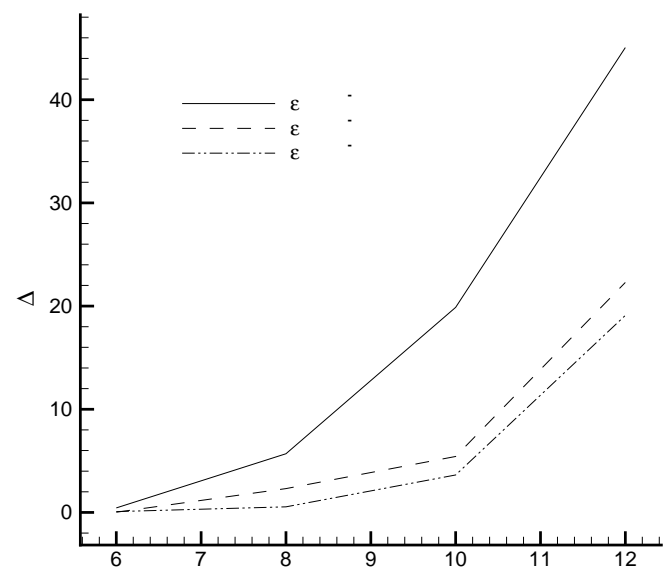

Figure 31: Time saved using the TE algorithm on the Kraichnan-Orszag problem with different threshold and different maximal levels. The coarsest level is equal to $m_{L}=4$ for all the computations. 


\subsection{Kraichnan-Orszag $1 D$ problem with a discontinuous unsteady pdf}

In this section, the 1D Kraichnan-Orszag problem presented in the previous section is solved employing a discontinuous unsteady probability distribution. In particular, the following pdf is retained

$$
p(\omega, t)= \begin{cases}p_{I} & \omega \leq \omega_{d} \\ p_{I I} & \omega>\omega_{d},\end{cases}
$$

where

$$
p_{I}= \begin{cases}N p_{I I} & 0 \leq t \leq \bar{t} \\ p_{I I} / N & \bar{t} \leq t \leq T\end{cases}
$$

in which $N=5, \bar{t}=10, T=30$ and $\omega_{d}(t)=\frac{11}{2500} t^{2}-\frac{11}{200} t+\frac{1}{3}$. Obviously, the normalization condition $\int_{\Xi} p(\xi) \mathrm{d} \xi=1$ is satisfied at each time step.

In figure 32(a), a contour of the function $p=p(\omega, t)$ is reported. The discontinuity has a parabolic shape and it disappears at nearly $t=21$. At $t=10$, the inversion between the left and right part of the discontinuity occurs creating a discontinuity also in time. After the disappearance of the discontinuity, the function reduces to a uniform classical distribution .

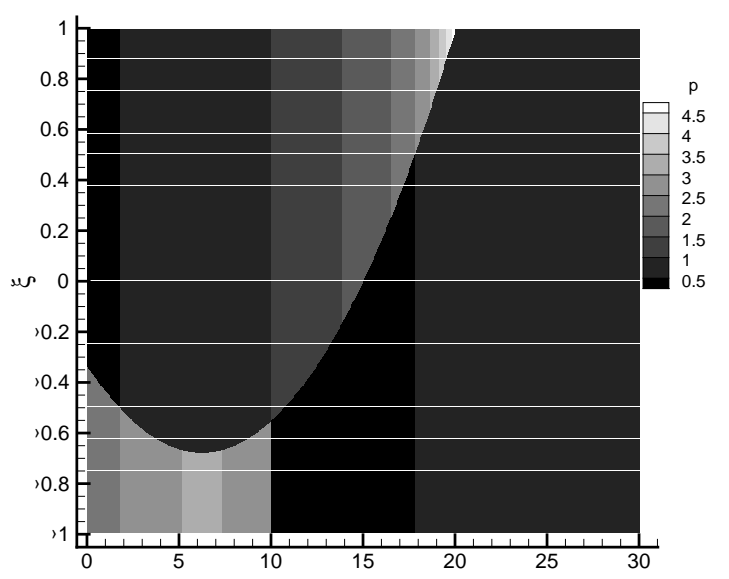

(a)

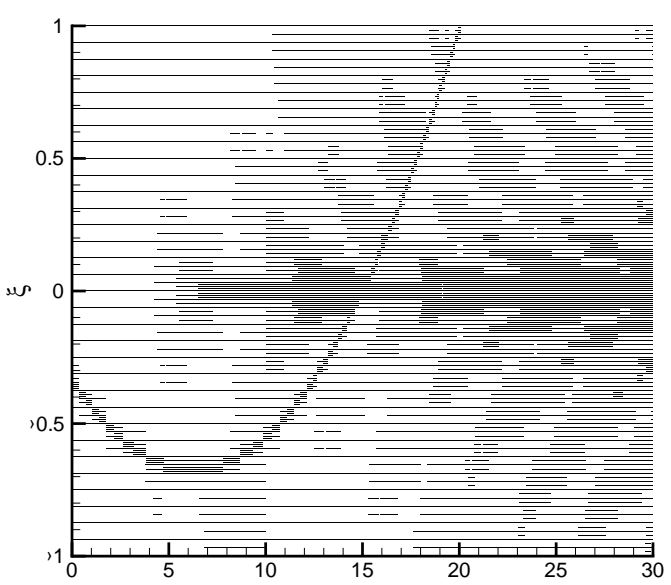

(b)

Figure 32: Probability density function (a) of the equation (36) and point distribution with TE $\left(\varepsilon=10^{-1}, m_{L}=4\right.$ and $\left.m_{\max }=8\right)(\mathrm{b})$.

The solutions for the statistics of the three variables $y_{1}, y_{2}$ and $y_{3}$ are reported in figure 33 . In figure 33 , the exact solution obtained with a MC with $N_{\xi}=20 \times 10^{6}$ is reported as reference. The results obtained with the TE algorithm with $\varepsilon=10^{-1}, m_{L}=4$ and $m_{\max }=8$, corresponding to a number of points equal to $N_{\xi}=88$, are compared to MC and PC results, computed with the same number of $N_{\xi}$.

In this case of unsteady pdf, the point distribution is affected by the presence of a moving discontinuity as well as the high gradients generated by the system responses. This creates a different distribution of points that becomes equal to the previous one (the uniform case) in the last part of the computation. The evolution of points distribution in time is reported in figure 32 (b).

Even in the case of discontinuous pdf, the variance for all the variables starts from zero and the mean of the variables $y_{2}$ and $y_{3}$ could assume some zero-values. For this reason, all the normalized errors are computed for time intervals equal to $[8,30]$, as done previously for uniform distributions.

In the figure 34 , the errors of the mean of the variable $y_{1}$ are reported measured in the $L_{1}, L_{2}$ and $L_{\infty}$ spaces. 
In figures 35, 36 and 37, the error norms of the variance (computed in the time interval [8,30], in the $L_{1}, L_{2}$ and $L_{\infty}$ spaces) are reported for $y_{1}, y_{2}$ and $y_{3}$, respectively. For all the simulations the TE algorithm shows better results with respect both MC and PC. In particular a lower error is reached for all the norms for each variable. For all the variable, in the case of $L_{1}$ and $L_{\infty}$ norms the error reached by the TE algorithm is up to an order of magnitude inferior to the correspondent errors, at the same number of points, by the MC and PC methods.

The evolution of points in time is reported in figure 38. Remark the increase of the number of points with respect to the points of the uniform distributed case, reported in figure 23(b). Remark also the different local behavior in the two cases. 


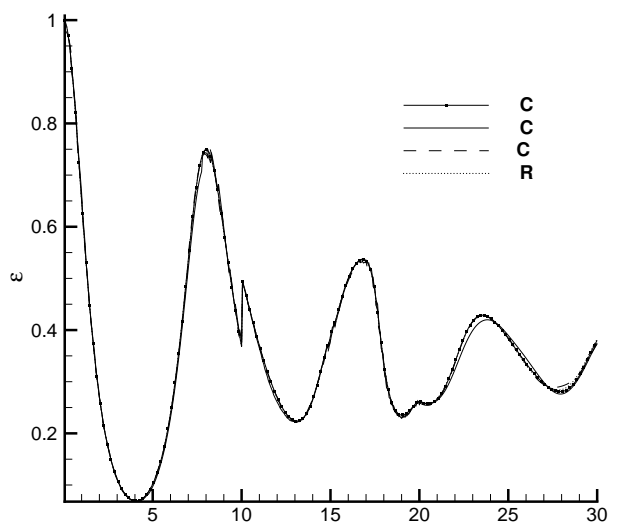

(a)

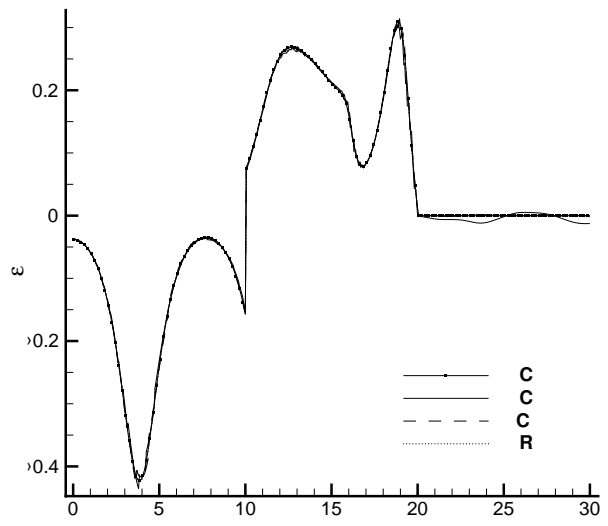

(c)

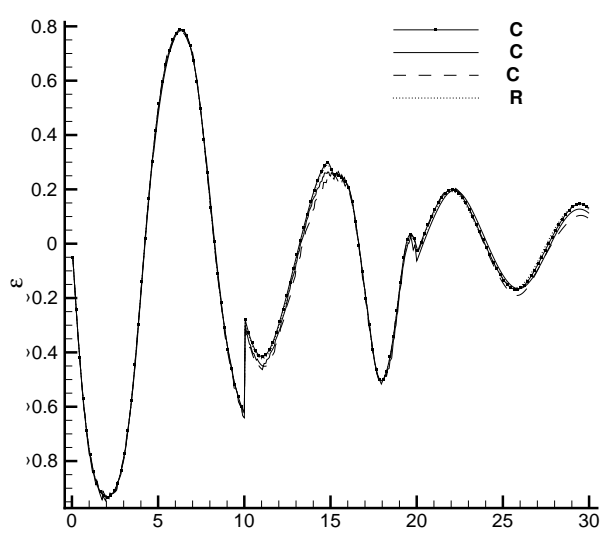

(e)

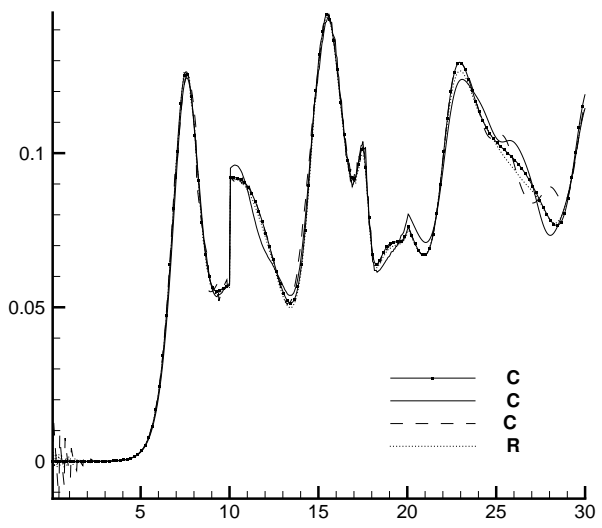

(b)

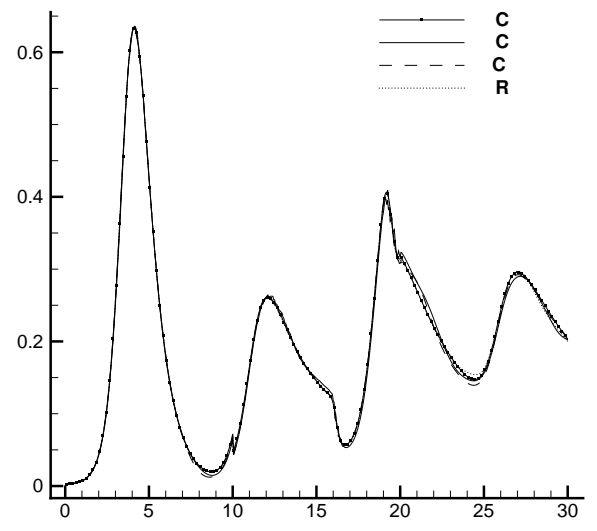

(d)

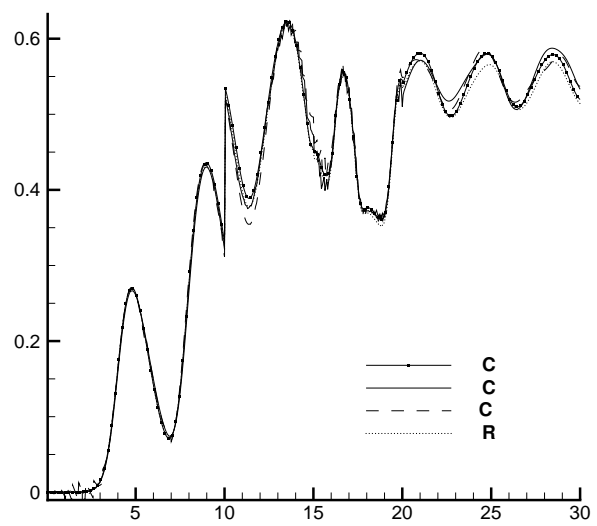

(f)

Figure 33: Time-evolutions of the mean (left) and variance (right) of the three variables $y_{1}$ (up), $y_{2}$ (middle) and $y_{3}$ (bottom) for the 1D KraichnanOrszag problem with the unsteady pdf of the equation (36).

RR n ${ }^{\circ} 7967$ 


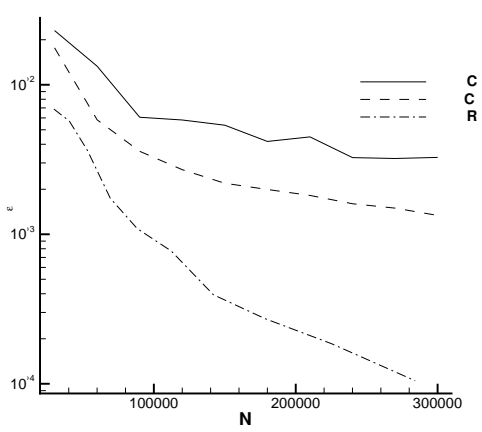

(a)

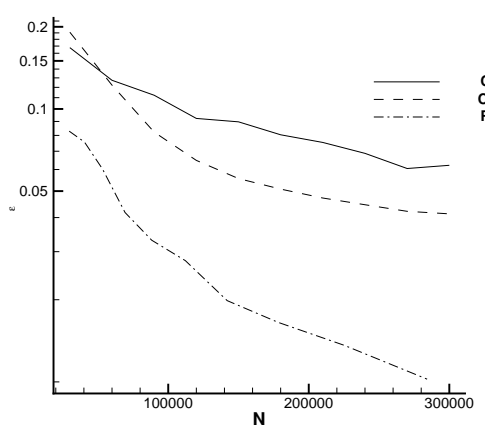

(b)

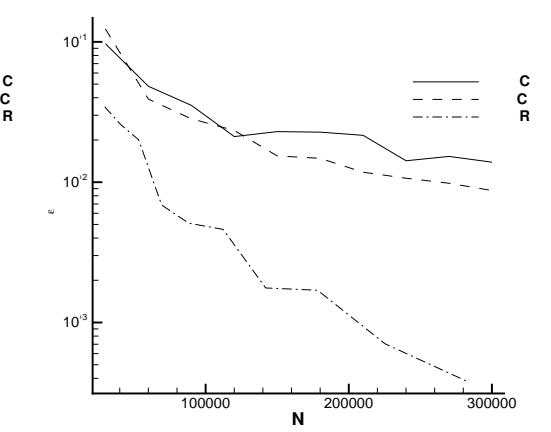

(c)

Figure 34: Error norms of the mean of the variable $y_{1}$ for the 1D Kraichnan-Orszag with unsteady pdf problem in the $L_{1}$ (a), $L_{2}$ (b) and $L_{\infty}$ (c) spaces

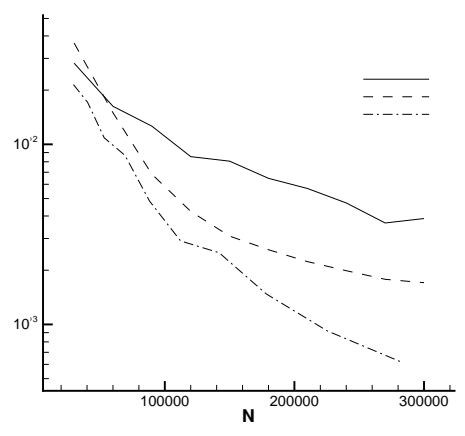

(a)

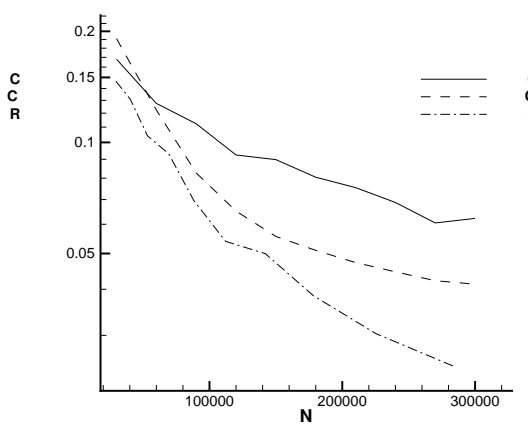

(b)

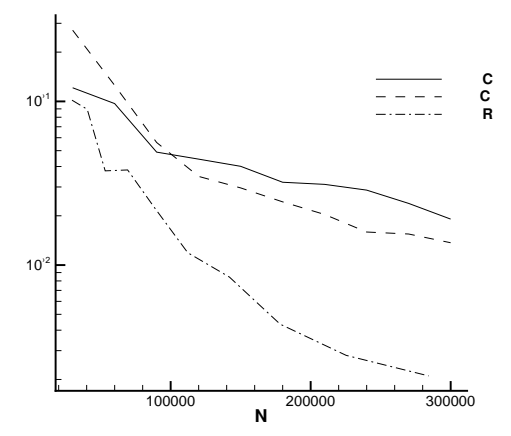

(c)

Figure 35: Error norms of the variance of the variable $y_{1}$ for the 1D Kraichnan-Orszag problem with unsteady pdf in the $L_{1}\left(\right.$ a), $L_{2}$ (b) and $L_{\infty}$ (c) spaces.

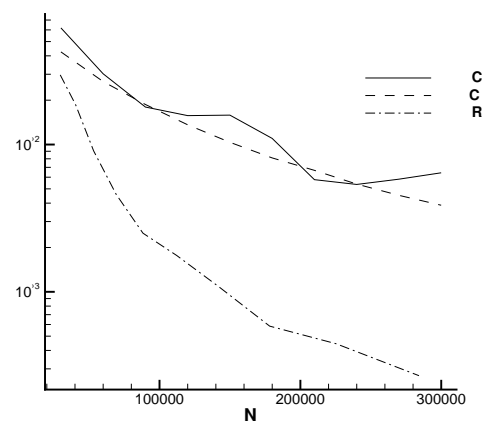

(a)

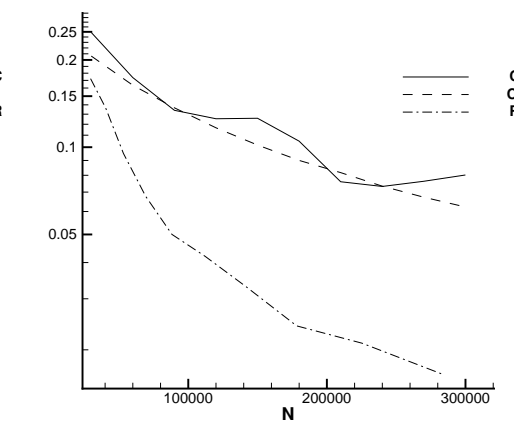

(b)

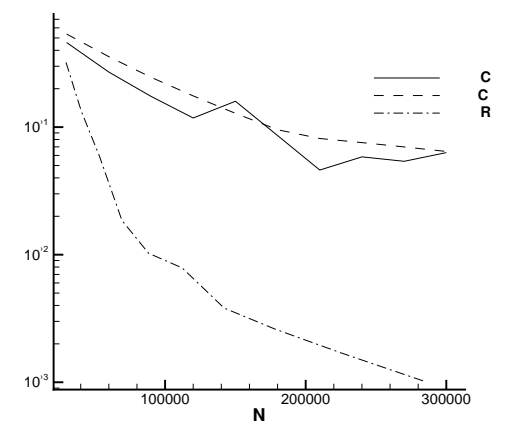

(c)

Figure 36: Error norms of the variance of the variable $y_{2}$ for the 1D Kraichnan-Orszag problem with unsteady pdf in the $L_{1}\left(\right.$ a), $L_{2}$ (b) and $L_{\infty}$ (c) spaces.

RR n ${ }^{\circ} 7967$ 


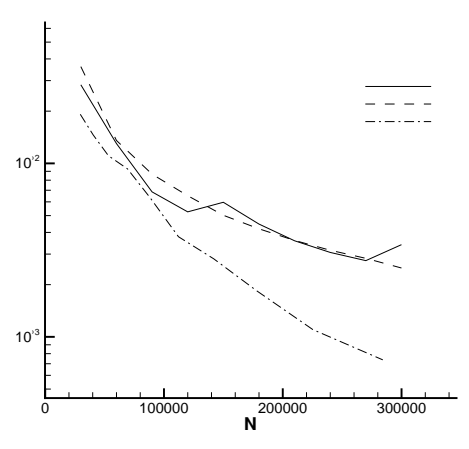

(a)

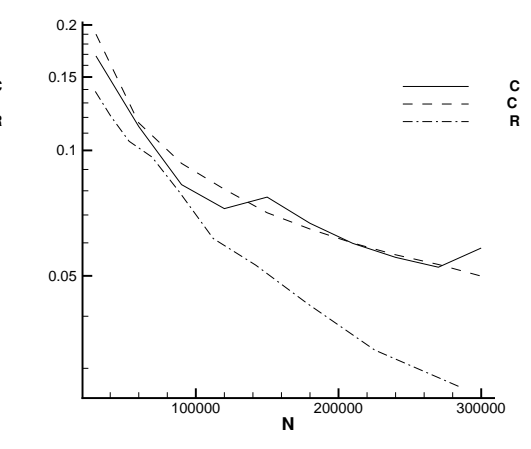

(b)

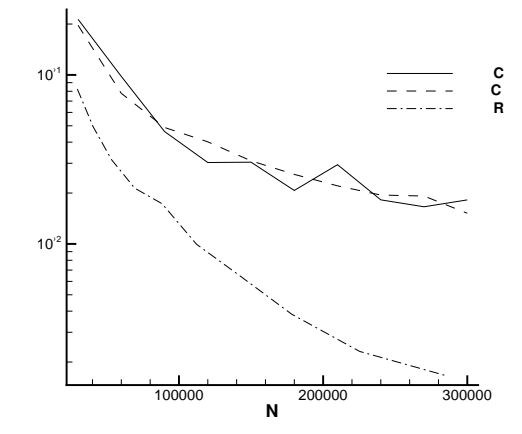

(c)

Figure 37: Error norms of the variance of the variable $y_{3}$ for the 1D Kraichnan-Orszag problem with unsteady pdf in the $L_{1}$ (a), $L_{2}$ (b) and $L_{\infty}$ (c) spaces.

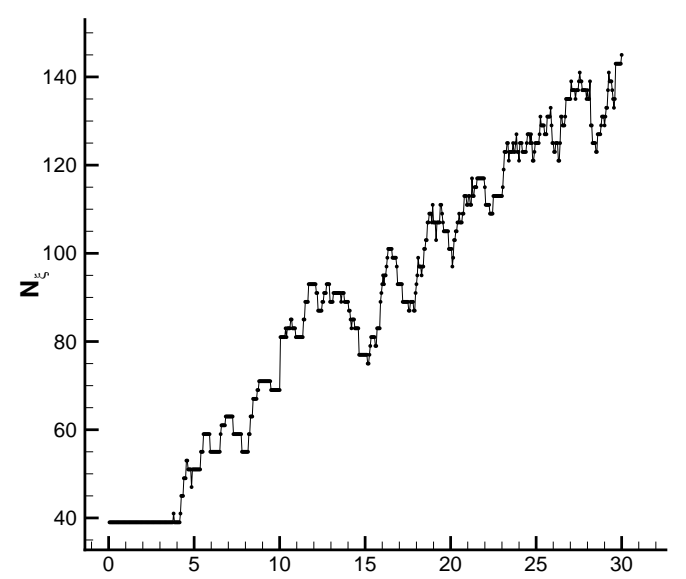

Figure 38: Evolution of the number of points in time for the TE algorithm with $\varepsilon=10^{-1}, m_{L}=4$ and $m_{\max }=8$, 1D Kraichnan-Orszag problem with discontinuous unsteady pdf. 


\subsection{A two degree-of-freedom subsonic airfoil model for aeroelasticity}

In this section, we present the results obtained with the TE algorithm to the motion of a typical wing section with two degree-of-freedom in a subsonic flight condition (see the figure 39 for a sketch of the problem and the convention adopted).

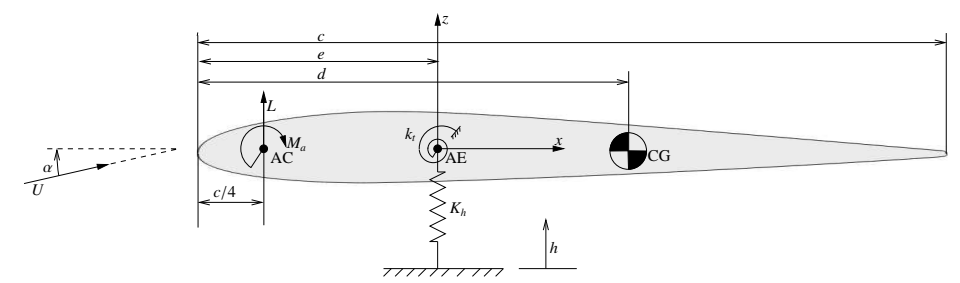

Figure 39: Sketch of the wing section aeroelastic problem with the forces and the convention adopted.

This simplified model can be employed to perform preliminary aeroelastic computations. The wing section is capable to reproduce the deflection and the torsional motion of a wing by two springs of stiffness $k_{h}$ and $k_{t}$ (see figure 39). The equations of motion can be derived by an energetic method as the Lagrangian approach. If a lift $L$ and an aerodynamic moment $M_{a}$ are applied to the wing section in the aerodynamic center $A C$ supposed to be located at the first quarter of the cord (in theory this assumption is valid only for the subsonic motion of a thin plate), the equations of motion, describing the traversal $h$ (in meters) and torsional $\alpha$ (in radiants) motion for a wing in flight at speed $U$ in air with density $\rho$, are as follows

$$
\left[\begin{array}{cc}
m & -S \\
-S & I
\end{array}\right]\left\{\begin{array}{c}
\ddot{h} \\
\ddot{\alpha}
\end{array}\right\}+\left[\begin{array}{cc}
k_{h} & 0 \\
0 & k_{t}
\end{array}\right]\left\{\begin{array}{l}
h \\
\alpha
\end{array}\right\}=\left\{\begin{array}{c}
L \\
e L+M_{a}
\end{array}\right\}
$$

in which $m$ is the total mass of the wing section, $S$ is the static inertia moment of the section computed as $S=m(d-e)$, $q$ is the dynamic pressure $q=1 / 2 \rho U^{2}$ and the polar inertia moment $I$ is obtained summing the moment referred to the center of gravity $I_{C G}$ and the transport contribution $m(d-e)^{2}$. The aerodynamics load are computed by means of a simplified quasi-steady strip theory in which the influence of the traversal velocity directly affects the angle-of-attack of the wing section but the memory effect is neglected, i.e. the aerodynamic loads are not dependent from the history of the fluid motion but only from the actual condition. Assuming a lift coefficient $C_{L 0}$, measured with respect to an incidence equal to zero, and a moment coefficient $C_{M}$, assumed to be independent from the angle-of-attack, the aerodynamics loads can be written as

$$
\begin{aligned}
& L=q c C_{L}=q c\left(C_{L 0}+C_{L / \alpha} \alpha_{e}\right) \\
& M_{a}=q c^{2} C_{M}
\end{aligned}
$$

in which the slope of the lift coefficient is indicated as $C_{L / \alpha}$ and the effective angle-of-attack $\alpha_{e}$ is relative to the local stream velocity

$$
\alpha_{e}=\alpha-\frac{\dot{h}}{U}
$$

Obviously, in the equation 38, the contribution to the lift due to $\alpha_{e}$ can be recasted to constitute the dumping matrix and can contribute to the stiffness matrix in the following final form

$$
\left[\begin{array}{cc}
m & -S \\
-S & I
\end{array}\right]\left\{\begin{array}{c}
\ddot{h} \\
\ddot{\alpha}
\end{array}\right\}+\left[\begin{array}{cc}
\frac{2 \pi q c}{U} & 0 \\
\frac{2 \pi q c e}{U} & 0
\end{array}\right]\left\{\begin{array}{l}
\dot{h} \\
\dot{\alpha}
\end{array}\right\}+\left[\begin{array}{cc}
k_{h} & -2 \pi q c \\
0 & k_{t}-2 \pi q c e
\end{array}\right]\left\{\begin{array}{l}
h \\
\alpha
\end{array}\right\}=\left\{\begin{array}{c}
q c C_{L 0} \\
q c e C_{L 0}+q c^{2} C_{M}
\end{array}\right\} .
$$

It was assumed that the slope of the $C_{L}-\alpha$ curve is equal to $2 \pi$ as demonstrated in the classical thin airfoil theory. This assumption is valid for small

angle-of-attack, i.e. roughly speaking between $-10^{\circ}$ and $10^{\circ}$. This condition is satisfied in the numerical tests reported below.

$\operatorname{RR} n^{\circ} 7967$ 


\begin{tabular}{|c|c|}
\hline \multicolumn{2}{|c|}{ Geometrical properties } \\
\hline$c[m]$ & 1 \\
\hline$e[m]$ & 0.2 \\
\hline$d[m]$ & 0.35 \\
\hline \multicolumn{2}{|c|}{ Structural properties } \\
\hline$k_{h}\left[N / m^{2}\right]$ & 1480 \\
\hline$k_{\alpha}[N / \mathrm{rad}]$ & 1000 \\
\hline \multicolumn{2}{|c|}{ Aerodynamics properties } \\
\hline$C_{L}[-]$ & 0.1 \\
\hline$C_{M}[-]$ & -0.001 \\
\hline \multicolumn{2}{|c|}{ Flight conditions } \\
\hline$U[\mathrm{~m} / \mathrm{s}]$ & 30 \\
\hline$\rho\left[\mathrm{Kg} / \mathrm{m}^{3}\right]$ & 1.2 \\
\hline
\end{tabular}

Table 4: Physical characteristics and flight conditions for the wing section.

In the table 4, all the numerical values employed to perform the numerical tests are reported. All the forces are expressed for unit span.

A parameterization of the mass properties of the section are chosen as follows:

$$
\begin{array}{ll}
m & =\xi \\
S & =m(d-e) \\
I_{C G} & =\xi^{2} c^{2}+d(d-e)^{2}
\end{array}
$$

where the random parameter $\xi=200 \omega+200[\mathrm{Kg} / \mathrm{m}]$ in which $\omega$ is distributed in $[0,1]$ with the following probability density function

$$
p= \begin{cases}6 / 5 & \text { for } \quad \omega<\frac{1}{3} \\ 3 / 2 & \text { for } \quad \frac{1}{3} \geq \omega \leq \frac{2}{3} \\ 3 / 10 & \text { for } \quad \omega>\frac{2}{3} .\end{cases}
$$

This kind of parameterization influences the mass matrix and then modifies the oscillation frequency of the system; the parameters (see table 4) are chosen in order to have a stable system, i.e. the system after the impulsive start oscillates entering in a transitory state and after some cycles reaches another equilibrium state. Of course, for lower parameters of the parameter $\xi$, the oscillation frequency is greater and the vice versa occurs for greater value. In fact, the oscillatory frequency $f[\mathrm{~Hz}]$ is proportional to root mean square of the ratio between the stiffness of the system and its mass.

The equation (41) can be written as

$$
M \ddot{\mathbf{x}}+C \dot{\mathbf{x}}+K \mathbf{x}=\mathbf{Q},
$$

where $M, C$ and $K$ are the mass, dumping and stiffness matrix, respectively. The unknown vector is $\mathbf{x}=\{h, \alpha\}^{\mathrm{T}}$ while the external forces are collected in the vector $\mathbf{Q}=\left\{q c C_{L 0}, q c\left(e C_{L 0}+c C_{M}\right)\right\}^{\mathrm{T}}$.

An explicit time discretization is applied, with $N_{t}$ constant time steps $t_{n} \in[0, T]$ where $t_{n}=n \Delta t$

$$
M \frac{\mathbf{x}_{n}-2 \mathbf{x}_{n-1}+\mathbf{x}_{n-2}}{\Delta t^{2}}+C \frac{\mathbf{x}_{n}-\mathbf{x}_{n-1}}{\Delta t}+K \mathbf{x}_{n}=\mathbf{Q}_{n} .
$$

This formulation leads to

$$
\left(\frac{1}{\Delta t^{2}} M+\frac{1}{\Delta t} C+K\right) \mathbf{x}_{n}-\left(\frac{2}{\Delta t^{2}} M+\frac{1}{\Delta t} C\right) \mathbf{x}_{n-1}+\frac{1}{\Delta t^{2}} M \mathbf{x}_{n-2}=\mathbf{Q}_{n} .
$$


from which the actual value of the unknown vector $\mathbf{x}\left(t_{n}\right)=\mathbf{x}_{n}$ can be computed explicitly from $\mathbf{x}_{n-1}$ and $\mathbf{x}_{n-2}$ as

$$
\mathbf{x}_{n}=\left(\frac{1}{\Delta t^{2}} M+\frac{1}{\Delta t} C+K\right)^{-1}\left(\left(\frac{2}{\Delta t^{2}} M+\frac{1}{\Delta t} C\right) \mathbf{x}_{n-1}-\frac{1}{\Delta t^{2}} M \mathbf{x}_{n-2}+\mathbf{Q}_{n}\right)
$$

Even in this case the TE algorithm is very straightforward to apply. However, attention must be devoted to the interpolation of both the term $\mathbf{x}_{n-1}$ and $\mathbf{x}_{n-2}$ in order to compute the actual value of $\mathbf{x}_{n}$, as already done in the case of the examples reported above, but only for one value at the previous time step. The two values of $x_{n-1}\left(\xi_{j}^{k}\right)$ and $x_{n-2}\left(\xi_{j}^{k}\right)$ are approximated by interpolation (here linear) obtaining

$$
\begin{aligned}
& \mathbf{x}_{n-1}\left(\xi_{j}^{k}\right) \approx \mathcal{I}\left(\xi_{j}^{k} ; \mathbf{x}^{k}\left(t_{n-1}\right)\right) \\
& \mathbf{x}_{n-2}\left(\xi_{j}^{k}\right) \approx \mathcal{I}\left(\xi_{j}^{k} ; \mathbf{x}^{k}\left(t_{n-2}\right)\right) .
\end{aligned}
$$

As shown in the section $\S 4$, the left and right values, with respect to the maximal level reached at each time step, should be used. So, in this case, the left value $\xi_{j_{L}}^{\bar{k}\left(t_{n-1}\right)}$ and the right one $\xi_{j_{R}}^{\bar{k}\left(t_{n-1}\right)}$ are considered for the time $t_{n-1}$ with respect the point $\xi_{j}^{k}\left(t_{n}\right)$ on the maximum level $\bar{k}\left(t_{n-1}\right)$ reached at the time $t_{n-1}$. As a consequence, the same is done at the time $t_{n-2}$. We remark here that in the general case $\bar{k}\left(t_{n-1}\right) \neq \bar{k}\left(t_{n-2}\right)$. The following steps are performed in order to interpolate the value of $\mathbf{x}\left(\xi_{j}^{k}\right)$ at the two time levels $t_{n-1}$ and $t_{n-2}$

- For both time levels, the left and right values are determined as:

$$
\begin{aligned}
& \xi_{j_{L}}^{\bar{k}\left(t_{n-1}\right)}\left(t_{n-1}\right)=\max \left\{\xi_{j}^{\bar{k}\left(t_{n-1}\right)} \mid \xi_{j}^{\bar{k}\left(t_{n-1}\right)}<\xi_{j}^{k}\right\} \\
& \xi_{j_{L}}^{\bar{k}\left(t_{n-2}\right)}\left(t_{n-2}\right)=\max \left\{\xi_{j}^{\bar{k}\left(t_{n-2}\right)} \mid \xi_{j}^{\bar{k}\left(t_{n-2}\right)}<\xi_{j}^{k}\right\}
\end{aligned}
$$

- For both the time levels the right values are determined as

$$
\begin{aligned}
& \xi_{j_{R}}^{\bar{k}\left(t_{n-1}\right)}\left(t_{n-1}\right)=\xi_{j_{L}}^{\bar{k}\left(t_{n-1}\right)}\left(t_{n-1}\right)+h_{\bar{k}\left(t_{n-1}\right)} \\
& \xi_{j_{R}}^{\bar{k}\left(t_{n-2}\right)}\left(t_{n-2}\right)=\xi_{j_{L}}^{\bar{k}\left(t_{n-2}\right)}\left(t_{n-2}\right)+h_{\bar{k}\left(t_{n-2}\right)}
\end{aligned}
$$

- The values of $\mathbf{x}\left(\xi_{j}^{k}\right)\left(t_{n-1}\right)$ and $\mathbf{x}\left(\xi_{j}^{k}\right)\left(t_{n-2}\right)$ are interpolated

$$
\begin{aligned}
\mathbf{x}\left(\xi_{j}^{k}, t_{n-1}\right) & \approx \mathcal{I}\left(\xi_{j}^{k} ; \mathbf{x}^{k}\left(t_{n-1}\right)\right) \\
& =\frac{\mathbf{x}\left(\xi_{j_{R}}^{\bar{k}\left(t_{n-1}\right)}, t_{n-1}\right)-\mathbf{x}\left(\xi_{j_{L}}^{\bar{k}\left(t_{n-1}\right)}, t_{n-1}\right)}{h_{\bar{k}\left(t_{n-1}\right)}} \xi_{j}^{k}+\mathbf{x}\left(\xi_{j_{L}}^{\bar{k}\left(t_{n-1}\right)}, t_{n-1}\right)-j_{L}\left(t_{n-1}\right)\left(\mathbf{x}\left(\xi_{j_{R}}^{\bar{k}\left(t_{n-1}\right)}, t_{n-1}\right)-\mathbf{x}\left(\xi_{j_{L}}^{\bar{k}\left(t_{n-1}\right)}, t_{n-1}\right)\right) \\
\mathbf{x}\left(\xi_{j}^{k}, t_{n-2}\right) & \approx \mathcal{I}\left(\xi_{j}^{k} ; \mathbf{x}^{k}\left(t_{n-2}\right)\right) \\
& =\frac{\mathbf{x}\left(\xi_{j_{R}}^{\bar{k}\left(t_{n-2}\right)}, t_{n-2}\right)-\mathbf{x}\left(\xi_{j_{L}}^{\bar{k}\left(t_{n-2}\right)}, t_{n-2}\right)}{h_{\bar{k}\left(t_{n-2}\right)}} \xi_{j}^{k}+\mathbf{x}\left(\xi_{j_{L}}^{\bar{k}\left(t_{n-2}\right)}, t_{n-2}\right)-j_{L}\left(t_{n-2}\right)\left(\mathbf{x}\left(\xi_{j_{R}}^{\bar{k}\left(t_{n-2}\right)}, t_{n-2}\right)-\mathbf{x}\left(\xi_{j_{L}}^{\bar{k}\left(t_{n-2}\right)}, t_{n-2}\right)\right),
\end{aligned}
$$

where the indexes $j_{L}\left(t_{n-1}\right)=\xi_{j_{L}}^{k\left(t_{n-1}^{-}\right)} / h_{\bar{k}\left(t_{n-1}\right)}$ and $j_{L}\left(t_{n-2}\right)=\xi_{j_{L}}^{k\left(t_{n-2}^{-}\right)} / h_{\bar{k}\left(t_{n-2}\right)}$. The expression obtained in the equation (51) inserted in the equation (47) allows recovering the final form of the numerical scheme.

In this numerical test, the section is assumed to be at rest, i.e. zero deflection and torsion with no transversal and angular velocity, and subject to impulsive start, i.e. $\mathbf{x}_{n-2}(\xi)=\mathbf{x}_{n-1}(\xi)=0$ for all $\xi \in \Xi$. Of course, this condition is equivalent to an impulsive start of the system that has no physical counterpart, but it represents, mathematically, a well-posed problem. The numerical tests are performed with a final time $T=18 \mathrm{~s}$ and a time step equal to $\Delta t=0.001$ chosen after a convergence study not reported here for brevity.

The time evolution of the variables $h$ and $\alpha$ is reported in figure 40. In the figure 40, the solution for MC, PC and the TE algorithm are reported with a number of point $N_{\xi}$, in the stochastic space, equal to 86 (equivalent to a 


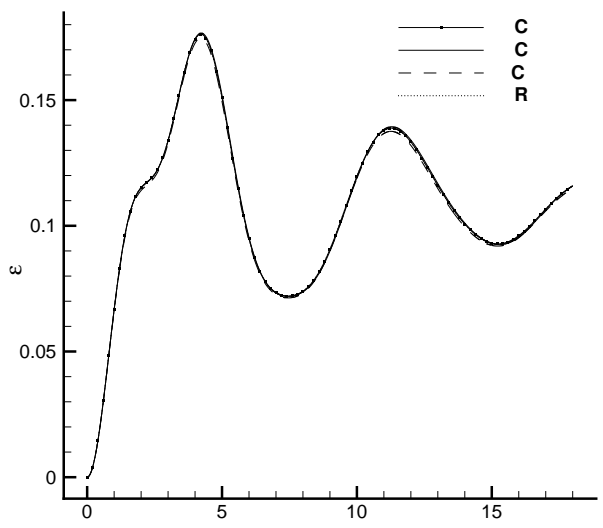

(a)

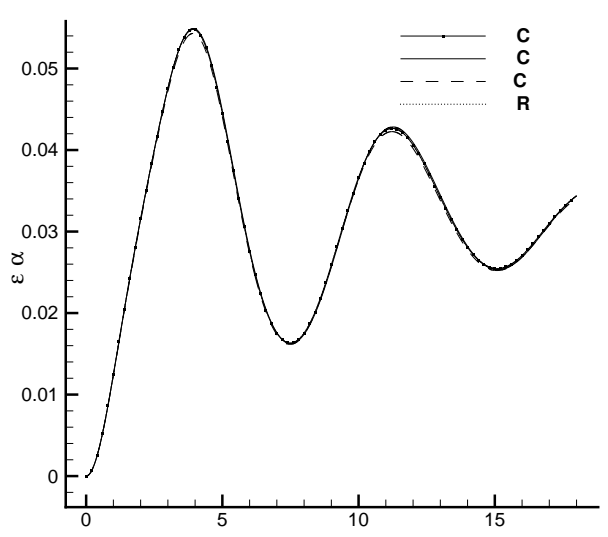

(c)

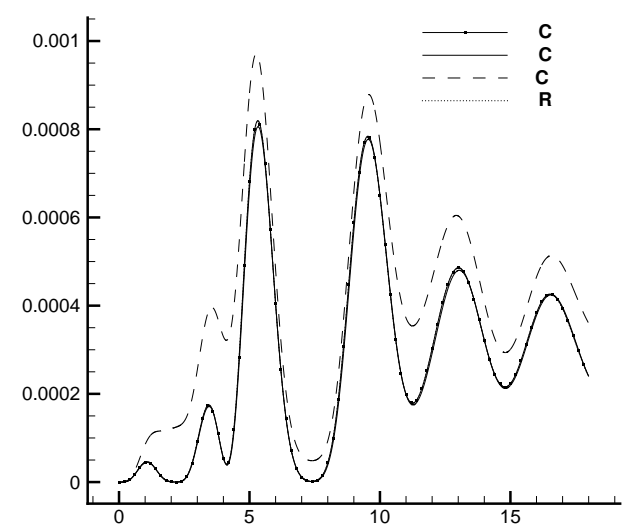

(b)

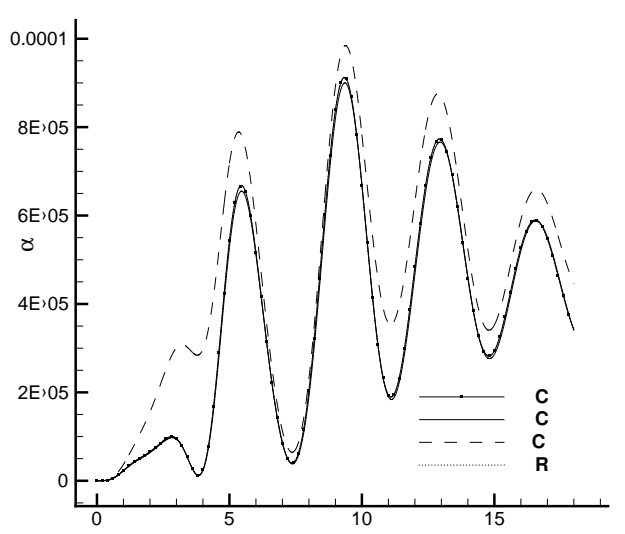

(d)

Figure 40: Time evolution of the mean (left) and variance (right) of the three variables $h$ (up), $\alpha$ (bottom) for the aeroelastic motion described by the equation (41).

TE algorithm of maximal level $m_{\max }=16$ with the coarsest level $m_{L}=3$ and a threshold equal to $\varepsilon=10^{-1}$ ). The reference solution obtained with a full converged Monte Carlo computation of $N_{\xi}=20 \times 10^{6}$ points in the stochastic space is also reported.

The mean value of $h$ and $\alpha$ computed by means of the three methods nearly coincide, but, for the variance, a stronger difference appears concerning the PC computations. Even if the shape of the evolution is well solved, the actual values are larger than the exact ones.

As already reported for the other numerical tests, the error of the mean and variance, measured in the $L_{1}, L_{2}$ and $L_{\infty}$ (see equations (32) and (33)), are computed for the three methods. In this case, the norms are computed for the time interval $[5,18]$, in order to avoid the normalization with values too close to zero, with a $N_{t}=18000$.

In figures 41 and 42, the errors for the mean and variance in norms $L_{1}, L_{2}$ and $L_{\infty}$ are reported for the variable $h$ and $\alpha$, respectively. In the figures, the total number of points in the grid $t-\omega$ is reported $N=N_{\xi} \times N_{t}$. The value ranges of $N_{\xi}$ ranges from 50 to 190 with a step of 20 for the MC, from degree 49 to 169 with step 20 for the PC. In order to compute different solutions employing the TE algorithm, the coarsest level is fixed as $m_{L}=3$ and the threshold equal to $\varepsilon=10^{-1}$, while the maximum allowed level, i.e. the finest level, is varied between 12 and 20 . 


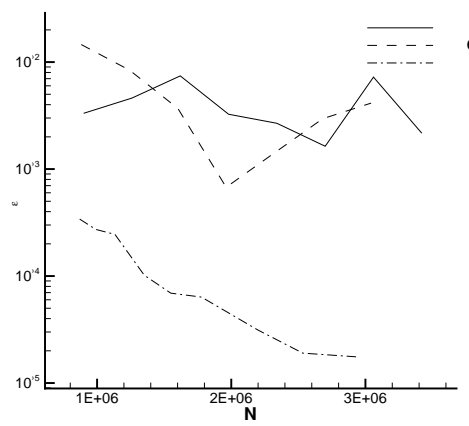

(a)

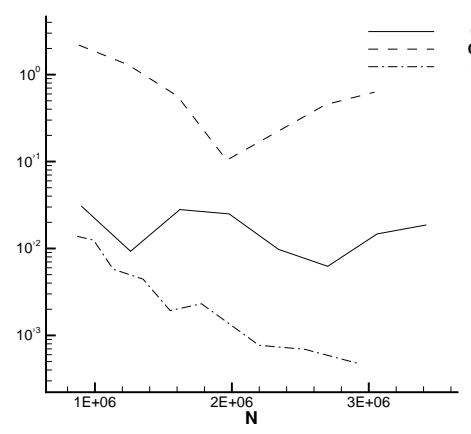

(d)

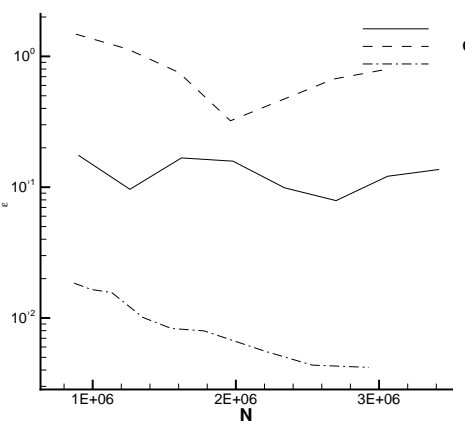

(b)

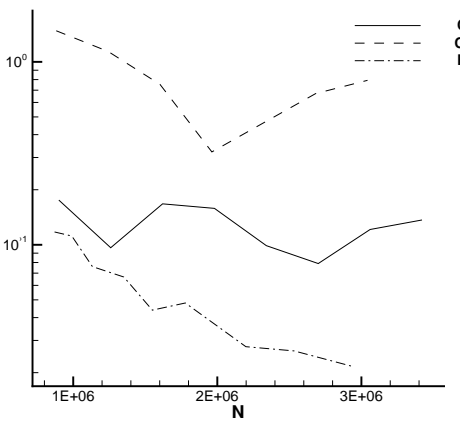

(e)

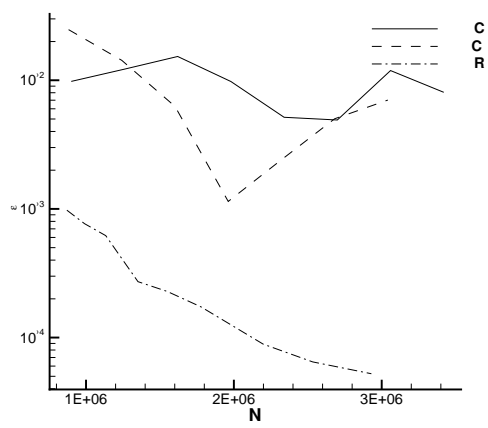

(c)

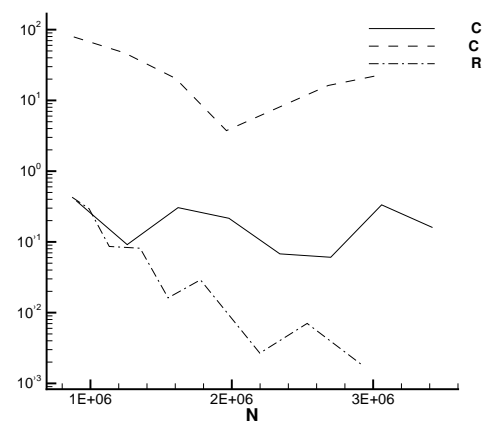

(f)

Figure 41: Error norms of the mean (top) and variance (bottom) of the variable $h$ of the aeroelastic problem in the $L_{1}$ ((a) and (d)), $L_{2}$ ((b) and (e)) and $L_{\infty}((\mathrm{c})$ and (f)) spaces.

All the numerical results display a superiority of the TE approach with respect to both the MC and PC in terms of level of error and convergence. In particular, all the error curves exhibit a smoother convergence with an error level lower than about an order of magnitude with respect to MC. Provided that the error on the mean remains of the same order of magnitude than MC, the PC shows larger errors for all the norms of the variance for both the variables, as already observed in figure 40. Finally, in the range of points considered, only the TE algorithm displays a good convergence behavior while both MC and PC exhibit a very poor rate of convergence and a too oscillatory pattern of the error.

Also in this case, the application of the TE algorithm exhibits good refinement/derefinement properties. For instance, the pattern of the points (figure 43(a)) and their number in time (figure 43(b)) are reported. Despite to a maximum number of points allowed equal to $N_{\xi}=2^{16}+1=65536$, the number of points remains always lower than 100 obtaining good compressing results (in term of evaluation compression).

\section{Conclusions}

In this work an innovative adaptive strategy for stochastic problem, the TE algorithm, inspired to the classical Harten's framework, is presented. A representation of the solution on a finest grid is computed starting from a coarsest grid, with a low number of evaluation of the function. Then, only a reduced set of point values, on the finest grid, is evaluated, while the remaining set is obtained by interpolation (from the previous levels). This procedure moves recursively, with a combination of interpolation and evaluation, from the coarsest level to the finest and from each time step to the successive one. At each time step, the scheme allows to recover the solution on the finest level with a one time scheme that embeds the encoding and the truncation procedures of the classical Harten framework. First, 


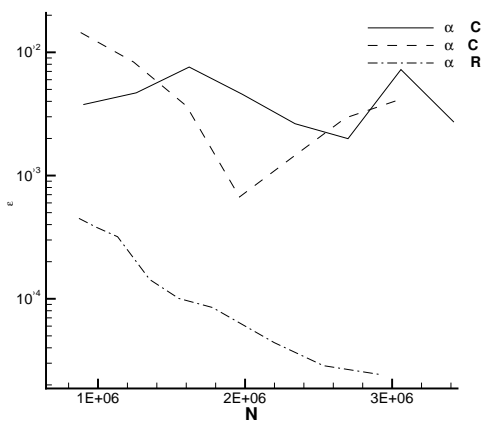

(a)

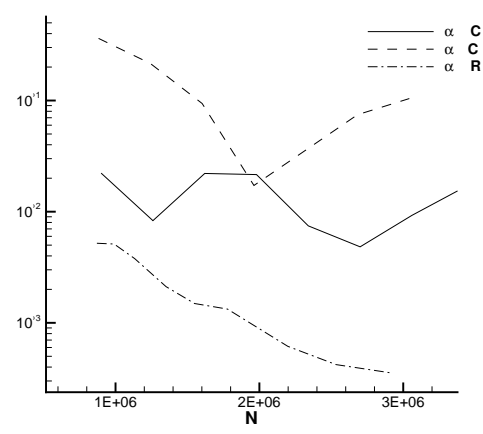

(d)

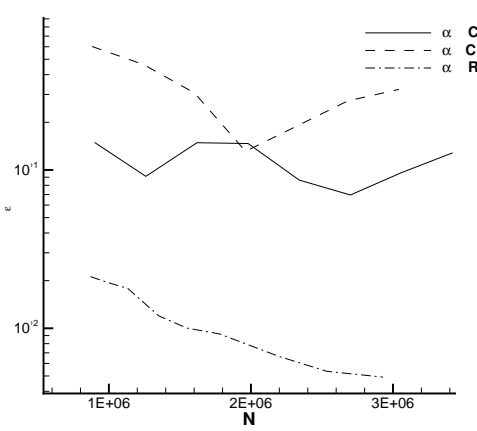

(b)

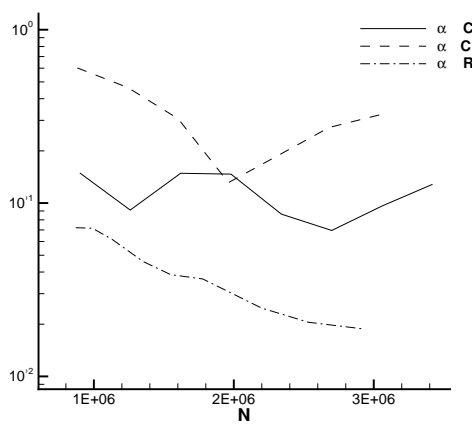

(e)

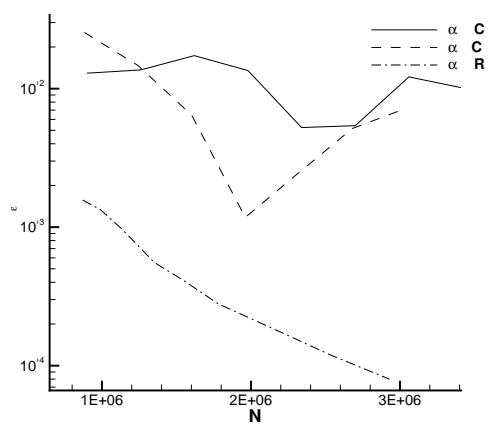

(c)

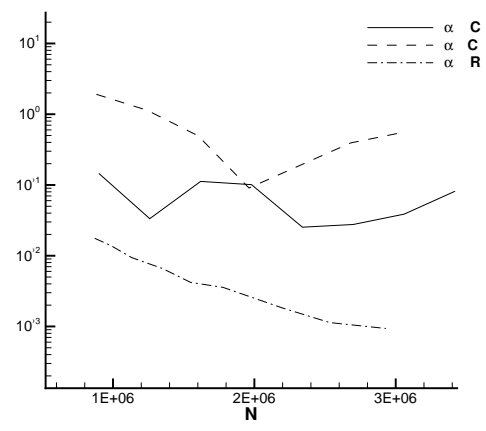

(f)

Figure 42: Error norms of the mean (top) and variance (bottom) of the variable $\alpha$ of the aeroelastic problem in the $L_{1}$ ((a) and (d)), $L_{2}$ ((b) and (e)) and $L_{\infty}((\mathrm{c})$ and (f)) spaces.

this basic algorithm is extended in order to solve a vectorial problem, i.e. computing the stochastic response of a system that has many outputs. Then, slight modifications are are suggested for unsteady problems. The TE algorithm must be, in the unsteady case, applied at each time step starting from the coarsest level to the finest one. According to the time-integration technique, the choice of a stencil in time is needed in order to evaluate a solution in the point of the time-stochastic space. The proposed time-advancement strategy can be easily used to build numerical scheme with automatic refinement/derefinement. The proposed formulations permits to recover the same results concerning the interpolation theory of the classical multiresolution approach, but with an extension to uncertainty quantification problems.

The interest of the present strategy is shown by performing several numerical problems where different forms of uncertainty distributions are taken into account, such as discontinuous and unsteady custom-defined probability density functions.

The TE algorithm is applied to several numerical test-cases in order to check its accuracy and convergence rate with respect to some classical stochastic methods, such as quasi-Monte Carlo and Polynomial Chaos. First, some steady algebraic problems are considered where analytical discontinuous functions are evaluated in terms of their expectancy and variance; for this case the performances in terms of compression and evaluation ratios are also evaluated. The TE algorithm displays a monotone decrease of the errors with respect to the number of evaluations. The capability of the TE algorithm to preserve accuracy for time-evolving solutions is displayed in two test-cases. The first-one deals with an ordinary differential equation (ODE), i.e. taken from [6] but with some modifications in order to obtain a more stiff $\xi-t$ pattern of the function.

In this case, the TE algorithm allows reaching a lower level of error at the same number of points. In the case of $L_{\infty}$ norm for the variance, at the same number of points it corresponds an order of magnitude of the error inferior up to 


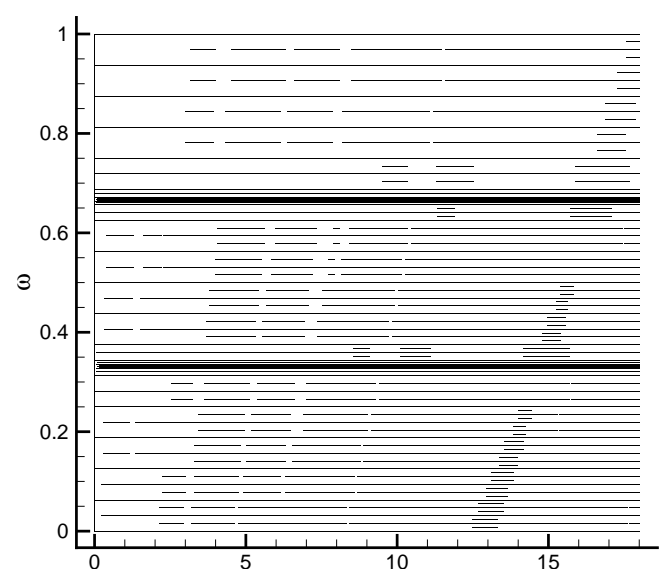

(a)

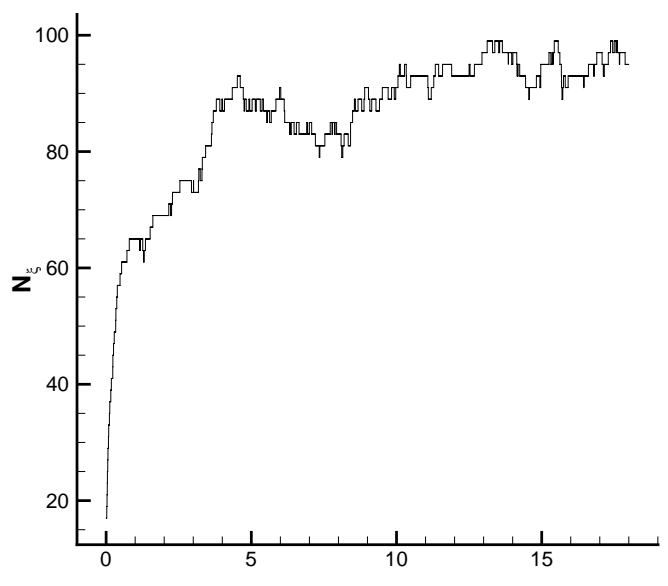

(b)

Figure 43: Patterns of the evaluations in the space $t-\omega((\mathrm{a}))$ and the number of points in the stochastic space $N_{\xi}$ employed by the TE algorithm with $\left.m_{\max }=16(\mathrm{~b})\right)$.

three orders of magnitude. The convergences exhibit the smoothest behaviors with respect to MC and PC despite to the presence of two discontinuities in the inputs. The observed behavior displays the refinement/derefinement capability of the algorithm to move and collocate points in the stochastic space with respect to the time evolution.

The second test-case for checking the convergence properties in long-time integration problem is the so-called Kraichnan-Orszag 1D ODE, a well-known problem in literature for testing UQ methods properties. Different kinds of pdf, i.e. uniform and discontinuous, are considered for both test-cases. For uniform distribution, TE displays better results in terms of accuracy and convergence of the solution with respect to MC and PC. In the case of long-time integration, the advantages of the application of the TE algorithm appear very poor with respect to the short-time case. Anyway, despite to the very high complexity of the solution, all the norms of the errors computed for each variable are smaller in the case of the application of the TE algorithm.

In the case of unsteady discontinuous pdf, the point distribution is affected by the presence of a moving discontinuity as well as the high gradients generated by the system responses. This creates a different distribution of points that reduces the global computational cost when using TE algorithm. As a consequence, performances of TE are very much better with respect to MC and PC solutions in terms of convergence.

Finally, a simplified model for aeroelastic study, a two degree-of-freedom typical wing section coupled with a quasi-steady strip theory model for aerodynamics, is used to compute the statistics of the motion considering uncertainties on mass properties for discontinuous probability distribution.

All the numerical results display a superiority of the TE approach with respect to both the MC and PC in terms of level of error and convergence. In particular, all the error curves exhibit a smoother convergence with an error level lower than about an order of magnitude with respect to MC. Provided that the error on the mean remains of the same order of magnitude than MC, the PC shows larger errors for all the norms of the variance for both the variables. Finally, in the range of points considered, only the TE algorithm displays a good convergence while both MC and PC exhibit a very poor rate of convergence and a too oscillatory pattern of the error.

\section{Acknowledgements}

Remi Abgrall has been partially supported by the ERC Advanced Grant ADDECCO N. 226316, while Gianluca Geraci has been fully supported by the ERC Advanced Grant ADDECCO N. 226316. The authors thank the support of the French Agence Nationale de la Recherche (ANR) under reference ANR-11-MONU-0008. 
[1] I. Graham, F. Kuo, D. Nuyens, R. Scheichl, I. Sloan, Quasi-Monte Carlo methods for elliptic PDEs with random coefficients and applications, Journal of Computational Physics 230 (2011) 3668-3694.

[2] N. Wiener, The Homogeneous Chaos, American Journal of Mathematics 60 (1938) 897-936.

[3] D. Xiu, G. E. Karniadakis, Modeling uncertainty in flow simulations via generalized polynomial chaos, Journal of Computational Physics 187 (2003) 137-167.

[4] X. Wan, G. E. Karniadakis, Beyond WienerAskey Expansions: Handling Arbitrary PDFs, Journal of Scientific Computing 27 (2005) 455-464.

[5] C. Soize, R. G. Ghanem, Physical systems with random uncertainties: chaos representations with arbitrary probability measure, SIAM Journal on Scientific Computing 26 (2004) 395-410.

[6] O. Le Maître, O. Knio, Spectral Methods for Uncertainty Quantification: With Applications to Computational Fluid Dynamics, Springer Verlag, 2010.

[7] R. G. Ghanem, P. D. Spanos, Stochastic Finite Elements. A spectral approach, Springer Verlag, 1991.

[8] X. Wan, G. E. Karniadakis, Long-term behavior of polynomial chaos in stochastic flow simulations, Computer Methods in Applied Mechanics and Engineering 195 (2006) 5582-5596.

[9] M. Gerritsma, J.-B. van der Steen, P. Vos, G. E. Karniadakis, Time-dependent generalized polynomial chaos, Journal of Computational Physics 229 (2010) 8333-8363.

[10] I. Babuška, F. Nobile, R. Tempone, A Stochastic Collocation Method for Elliptic Partial Differential Equations with Random Input Data, SIAM Review 52 (2010) 317.

[11] S. A. Smolyak, Quadrature and interpolation formulas for tensor products of certain classes of functions, Soviet Math. Dokl. (1963) 240-243.

[12] R. E. Bellman, B. Richard, Adaptive Control Processes: A Guided Tour, Princeton University Press, 1961.

[13] B. Ganapathysubramanian, N. Zabaras, Sparse grid collocation schemes for stochastic natural convection problems, Journal of Computational Physics 225 (2007) 652-685.

[14] M. Griebel, Sparse grids and related approximation schemes for higher dimensional problems, in: L. P. Todd, A. Pinkus, E. Suli, M.J. (Eds.), Foundations of Computational Mathematics (FoCM05), Santander, Cambridge University Press, 2006, pp. 106-161.

[15] J. Foo, G. E. Karniadakis, Multi-element probabilistic collocation method in high dimensions, Journal of Computational Physics 229 (2010) $1536-1557$.

[16] O. Le Maître, Uncertainty propagation using WienerHaar expansions, Journal of Computational Physics 197 (2004) $28-57$.

[17] O. Le Maître, Multi-resolution analysis of Wiener-type uncertainty propagation schemes, Journal of Computational Physics 197 (2004) $502-531$.

[18] J. a. S. Witteveen, G. Iaccarino, Simplex Elements Stochastic Collocation in Higher-Dimensional Probability Spaces, in: 51st AIAA/ASME/ASCE/AHS/ASC Structures, Structural Dynamics, and Materials Conference 12 - 15 April 2010, Orlando, Florida, April.

[19] N. Agarwal, N. Aluru, A domain adaptive stochastic collocation approach for analysis of MEMS under uncertainties, Journal of Computational Physics 228 (2009) 7662-7688.

[20] X. Ma, N. Zabaras, An adaptive hierarchical sparse grid collocation algorithm for the solution of stochastic differential equations, Journal of Computational Physics 228 (2009) 3084-3113.

[21] R. Abgrall, A simple, flexible and generic deterministic approach to uncertainty quantifications in non linear problems: application to fluid flow problems, Technical Report, 2007.

[22] R. Abgrall, P. M. Congedo, C. Corre, S. Galéra, A simple semi-intrusive method for uncertainty quantification of shocked flows, comparison with a non-intrusive polynomial chaos method, in: V European Conference on Computational Fluid Dynamics ECCOMAS CFD 2010 J. C. F. Pereira and A. Sequeira (Eds) Lisbon, Portugal,14-17 June 2010, June, pp. 1-17.

[23] R. Abgrall, P. M. Congedo, S. Galéra, G. Geraci, Semi-intrusive and non-intrusive stochastic methods for aerospace applications, in: 4TH EUROPEAN CONFERENCE FOR AEROSPACE SCIENCES, Saint Petersburg, Russia, July 4th-8th, 2011, 1, pp. 1-8.

[24] A. Harten, Multiresolution Representation of Data : A General Framework, SIAM Journal on Numerical Analysis 33 (1996) $1205-1256$.

[25] A. Harten, Multiresolution algorithms for the numerical solution of hyperbolic conservation laws, Communications on Pure and Applied Mathematics 48 (1995) 1305-1342.

[26] R. Abgrall, A. Harten, Multiresolution Representation in Unstructured Meshes, SIAM Journal on Numerical Analysis 35 (1998) $2128-2146$.

[27] A. Harten, Adaptive multiresolution schemes for shock computations, Journal of Computational Physics 135 (1994) $260-278$.

[28] S. Orszag, Dynamical properties of truncated Wiener-Hermite expansions, Physics of Fluids 10 (1967) $2603-2613$.

\section{Appendix A. Relation between the Lagrange interpolation and the average reconstruction in the case of con- stant reconstruction}

In this section we want to make explicit the relation between the Lagrangian interpolation and the average reconstruction procedure in the case of constant reconstruction. With reference to the mesh of $\Xi$ defined in the section 2 , in a natural way, it is possible to define a tessellation (of the generic $k$ th level) of $N_{k}+1$ cells $\left\{\Xi_{j}^{k}\right\}_{j=0}^{N_{k}}$, as

$$
\Xi_{j}^{k}=\left[\xi_{j-1 / 2}^{k}, \xi_{j+1 / 2}^{k}\right] \quad \text { with } \quad \Xi=\bigcup_{j=0}^{N_{k}} \Xi_{j}^{k},
$$

mutually independent with respect to a measure $\mu\left(\Xi_{j}^{k}\right)=\int_{\Xi_{j}^{k}} \mathrm{~d} \mu$, i.e.

$$
\mu\left(\Xi_{i}^{k} \cap \Xi_{j}^{k}\right)=0 \text { for } i \neq j
$$


If an interpolation operator $\mathcal{I}(\xi ; u)$ is chosen in order to approximate the function $u$ at the coordinate $\xi$, it is possible to define an average (conditional) value $\bar{u}_{j}^{k}$ on every cell $\Xi_{j}^{k}$ at the generic level $k$ th as

$$
\bar{u}_{j}^{k}=\frac{\int_{\Xi_{j}^{k}} I_{j}^{k}\left(\xi ; u^{k}\right) \mathrm{d} \mu}{\int_{\Xi_{j}^{k}} \mathrm{~d} \mu} .
$$

With such an operator, the values of the function (the average values) in the missing points, i.e. the points $\xi \in \mathcal{G}^{k-1}$ but not to $\mathcal{G}^{k} \cap \mathcal{G}^{k-1}$, can be obtained directly from the knowledge of the function at the level $k$ th

$$
\tilde{u}_{2 j-1}^{k-1}=\frac{\int_{\Xi_{2 j-1}^{k-1}} I_{2 j-1}^{k-1}\left(\xi ; u^{k}\right) \mathrm{d} \mu}{\int_{\Xi_{2 j-1}^{k-1}} \mathrm{~d} \mu} \text { with } \quad 1 \leq j \leq N_{k} .
$$

The operator $I_{2 j-1}^{k-1}\left(\xi ; u^{k}\right)$ can be expressed as a function only of the values of the function at the level $k$ th. The interpolation operator $\mathcal{I}_{j}^{k}\left(\xi ; u^{k}\right)$ on the cell $j$ at the level $k$ th could be chosen, as a polynomial of degree $r: P_{j}^{k} \in \mathbb{P}^{r}(\mathbb{R})$. If a proper stencil $\mathcal{S}_{j}^{k}$ is provided, the polynomial reconstruction is obtained imposing the condition

$$
\frac{\int_{\Xi_{j}^{k}} P_{j}^{k}(\xi) \mathrm{d} \mu}{\int_{\Xi_{j}^{k}} \mathrm{~d} \mu}=\bar{u}_{j}^{k}
$$

Once the interpolation operator is determined, from the knowledge of the function at the coarser level $k$ th, the point value $u=u(\xi)$ of the function in a cell $\left(\xi \in \Xi_{j}^{k-1}\right)$ can be obtained directly by evaluating $\mathcal{I}\left(\xi ; u^{k-1}\right)$.

Now let us suppose a first order reconstruction, i.e. a piecewise constant reconstruction. In this case, the stencil $S_{j}^{k}=\left\{\Xi_{j}^{k}\right\}$ and the value $\bar{u}_{2 j-1}^{k-1}$ can be approximated from (A.4) as (see figure A.44)

$$
\begin{aligned}
\tilde{u}_{2 j-1}^{k-1}=\frac{\int_{\Xi_{2 j-1}^{k-1}} I_{2 j-1}^{k-1}\left(\xi ; u^{k}\right) \mathrm{d} \mu}{\int_{\Xi_{2 j-1}^{k-1}} \mathrm{~d} \mu} & \approx \frac{1}{\int_{\xi_{2 j-3 / 2}^{k-1}}^{\xi_{2 j-1}^{k-1}} \mathrm{~d} \mu}\left(\bar{u}_{j-1}^{k} \int_{\xi_{2 j-3 / 2}^{k-1}}^{\xi_{2 j-1}^{k-1}} \mathrm{~d} \mu+\bar{u}_{j}^{k} \int_{\xi_{2 j-1}^{k-1}}^{\xi_{2 j-1 / 2}^{k-1}} \mathrm{~d} \mu\right) \\
& =\frac{1}{h_{k-1}}\left(\frac{1}{2}\left(\bar{u}_{j-1}^{k}+\bar{u}_{j}^{k}\right) h_{k-1}\right)=\frac{1}{2}\left(\bar{u}_{j-1}^{k}+\bar{u}_{j}^{k}\right)
\end{aligned}
$$

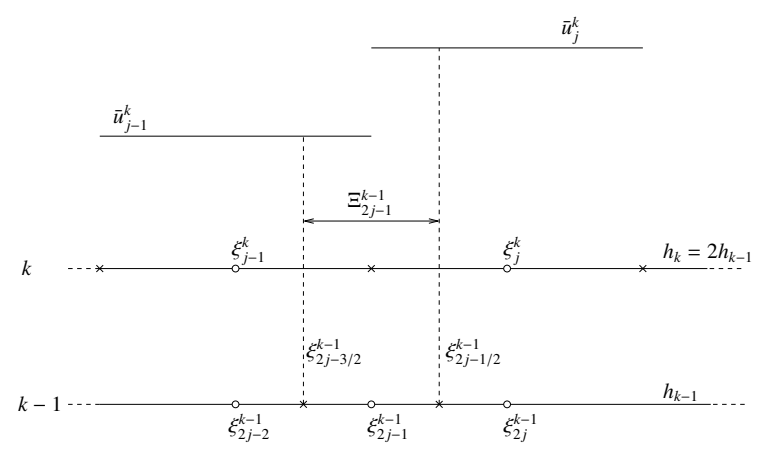

Figure A.44: Approximation of the value $\tilde{u}_{2 j-1}^{k-1}$ by means of a constant reconstruction on the cell $\Xi_{j-1}^{k}$ and $\Xi_{j}^{k}$. The circles mark the cell center while the crosses indicate the cell interfaces.

The equation (A.6) displays that in the case of constant approximation the reconstruction is equivalent to a linear interpolation between the two adjacent values of $u$ at the previous coarser level. 


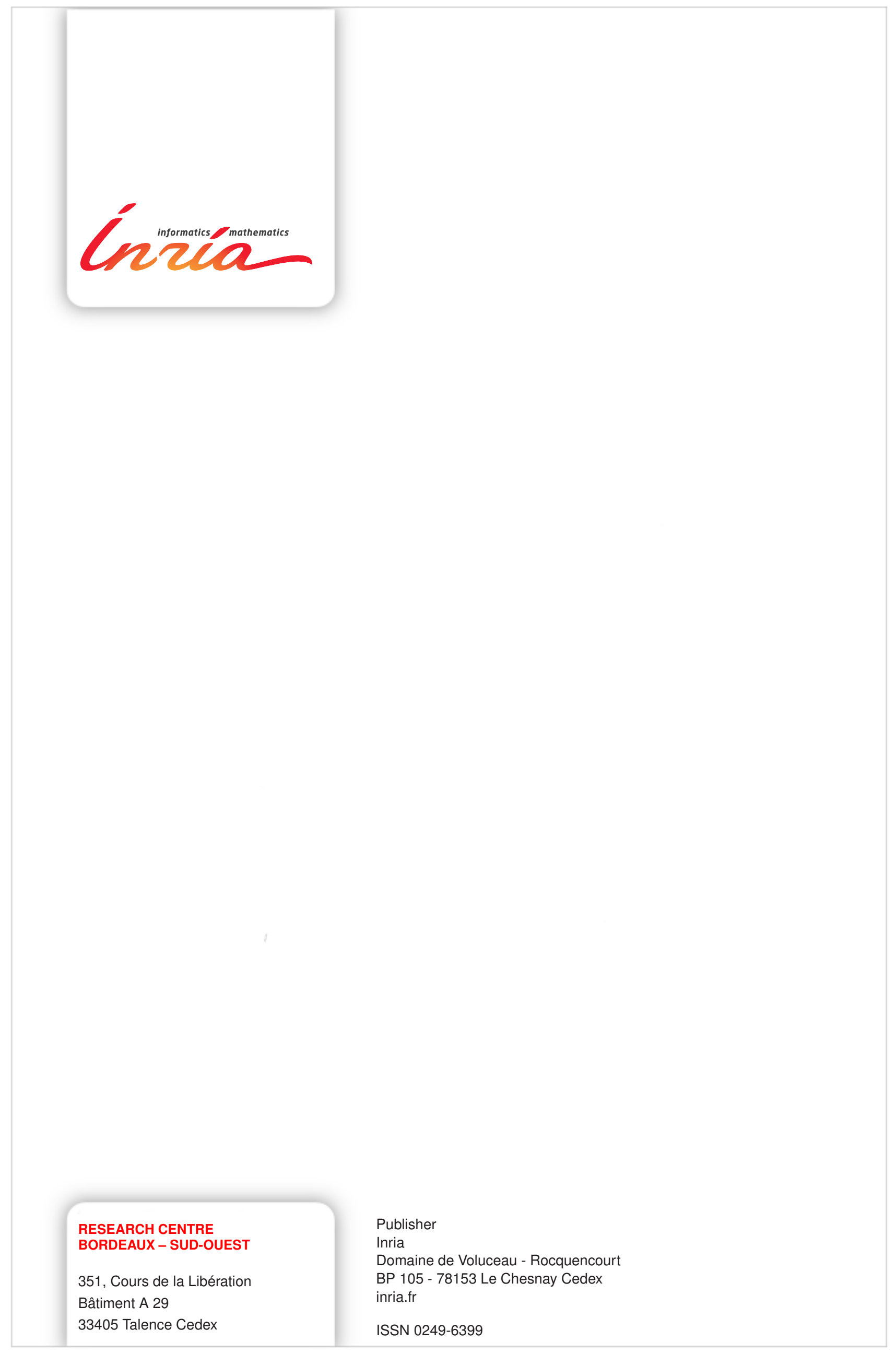

